\title{
THE DEGREE DISTRIBUTION AND THE NUMBER OF EDGES BETWEEN NODES OF GIVEN DEGREES IN DIRECTED SCALE-FREE GRAPHS
}

\author{
Evgeniy A. Grechnikov \\ Research Division, Yandex, Moscow, Russia
}

\begin{abstract}
In this article, we introduce our study of some important statistics of the random graph in the directed preferential attachment model introduced by B. Bollobás, C. Borgs, J. Chayes, and O. Riordan. First, we find a new asymptotic formula for the expectation of the number $n_{i n}(t, d)$ of nodes of a given in-degree $d$ in a graph in this model with $t$ edges, which covers all possible degrees. The out-degree distribution in the model is symmetrical to the in-degree distribution. Then we prove tight concentration for $n_{i n}(t, d)$ while $d$ grows up to the moment when $n_{i n}(t, d)$ decreases to $\ln ^{2} t$; if $d$ grows even faster, $n_{i n}(t, d)$ is zero whp.

Furthermore, we study an average number of edges from a vertex of out-degree $d_{1}$ to a vertex of in-degree $d_{2}$. In particular, we prove that it grows proportionally to $d_{1} d_{2} / t$ if

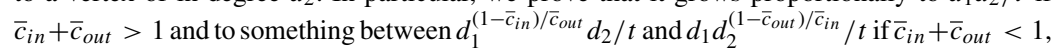
tending to the first expression when $d_{1}$ is small compared to $d_{2}$ and to the second one when $d_{1}$ is large; $\bar{c}_{i n}$ is such that the main term of $n_{i n}(t, d)$ is proportional to $d^{-1-1 / \bar{c}_{\text {in }} t}, \bar{c}_{\text {out }}$ is symmetrical for out-degrees. We also give exact formulas for intermediate cases.
\end{abstract}

\section{INTRODUCTION}

The real world has many interesting structures that can be thought of as graphs. A typical example is the World Wide Web: one can consider webpages to be vertices of a graph and hyperlinks to be edges. One of the productive methods for studying these graphs involves investigation of a suitable random graph model.

The first models of random graphs were constructed and investigated long ago. Classical models and results are systematized, for example, in [4] and [14]. However, they are not suitable for approximation of dynamically changing and nonuniform networks. In particular, the degree sequences of the graphs in these models are very far from those observed in reality.

Recently, other models of random graphs were constructed to more closely match the growth of real networks. One of the first descriptions of such a model is from [3]. The graph in this model grows sequentially in discrete steps. Each step adds one vertex and several edges that connect a new vertex with existing ones according to the "preferential attachment" rule: the probability of an existing vertex to receive a new edge depends on the current degree of this node, so more "popular" nodes are more attractive for new edges. In [3], the probability is proportional to the current degree; [3] gives some heuristic arguments

Address correspondence to Evgeniy Grechnikov, 16 Leo Tolstoy St., Moscow 119021, Russia. Email: grechnik@yandex-team.ru 
suggesting that the number of vertices with degree $d$ decreases in proportion to $d^{-3}$. The same quantity in real networks decreases proportionally to $d^{-\gamma}$ with different $\gamma$ for different networks, following the so called "power law." Later, an explicit model was proposed [6] based on the preferential attachment rule. The model of [6] resolves some ambiguities of [3]; also, [6] rigorously proves a theorem concerning degree sequence.

In [10] and [11], two groups of researchers independently proposed to add to the model one more parameter - an "initial attractiveness" of a node, which is a positive constant not depending on the degree. Equivalently, the probability in the proposed model is a linear function in the degree. Articles [10] and [11] use some heuristic arguments to show that the model allows obtaining the power law for degree sequence with any exponent less than -2. In [7], the model was formalized and the power law was rigorously proven when all the parameters are natural numbers and the degree grows slowly compared to the number of vertices. We analyzed this model in [12], removing the restriction on degree and considering also the quantity similar to $X\left(t, d_{1}, d_{2}\right)$ in the current work.

A quite general model was analyzed in [9]. There are two different procedures for updating the graph in this model: adding a new vertex with several edges and adding several edges to an already existing vertex. At every step, one of these procedures is selected at random, independently of other steps.

All models mentioned yield essentially an undirected graph; although edges have a natural direction, the out-degree sequence is unnatural. In particular, in models [6] and [7], all vertices have the same out-degree. Two models of directed graphs were suggested in [5] and [8]. Both models use three different procedures for updating the graph and select one of them at random for each step, independently. Both models mix preferential attachment with uniform random selection of source and target vertices, but the details of mixing are different: [5] follows [7] and assigns probabilities that are proportional to a linear shift of vertex degree, whereas [8] follows [9] and uses the weighted sum of probability of preferential attachment (that is proportional to vertex degree) and probability of uniform selection. Also, the model of [5] always creates a vertex with one edge (incoming or outgoing) in the name of simplicity, whereas the model of [8] allows an arbitrary finite distribution for the number of edges created with a new vertex in the name of generality. These models tend to give similar results, though.

\section{THE MODEL AND FORMULATION OF RESULTS}

We analyze the following model of a directed random graph introduced in [5]. The model allows multiple edges between same vertices and loops. Denote the in-degree of a vertex $v$ as $d_{\text {in }}(v)$ and the out-degree of a vertex $v$ as $d_{\text {out }}(v)$.

- There are 6 parameters $\alpha \in[0,1], \beta \in[0,1], \gamma \in[0,1], \alpha+\beta+\gamma=1, \delta_{\text {in }} \geq 0$, $\delta_{\text {out }} \geq 0$, and graph $G_{0}$.

- The graph $G_{0}$ should contain at least one vertex. If $\delta_{\text {in }}=0$ or $\delta_{\text {out }}=0$, it should also contain at least one edge.

- There is also time $t \in \mathbb{Z}, t \geq t_{0}$, where $t_{0}$ is the number of edges in $G_{0}$. The model defines a separate probability space for every possible moment of time; we denote the probability space at time $t$ as $\mathcal{G}(t)$.

- The probability space $\mathcal{G}\left(t_{0}\right)$ contains only one graph $G_{0}$.

- Given a random graph $G$ at time $t$, a random graph at time $t+1$ is constructed from $G$ by one of the following processes. Let $n$ be the number of vertices in $G$. 
$\dagger$ With probability $\alpha$, add a new vertex $v$ and an edge from $v$ to one of vertices in $G$. The target vertex is selected randomly, a vertex $w \in G$ is selected with probability $\frac{d_{i n}(w)+\delta_{\text {in }}}{t+\delta_{\text {in }} n}$.

†† With probability $\beta$, add an edge from a random vertex $v \in G$ to a random vertex $w \in G$. A vertex $v$ is selected with probability $\frac{d_{\text {out }}(v)+\delta_{\text {out }}}{t+\delta_{\text {out }} n}$, a vertex $w$ is selected independently of $v$ with probability $\frac{d_{i n}(w)+\delta_{i n}}{t+\delta_{i n} n}$.

$\ddagger$ With probability $\gamma$, add a new vertex $w$ and an edge from one of vertices in $G$ to $w$. The source vertex is selected randomly, a vertex $v \in G$ is selected with probability $\frac{d_{\text {out }}(v)+\delta_{\text {out }}}{t+\delta_{\text {out }} t}$.

- It is easy to see that a random graph at time $t$ has exactly $t$ edges and a random number of vertices concentrated around $(\alpha+\gamma) t$.

We assume that $\alpha+\gamma>0$; otherwise, the number of vertices would not change over time.

We consider parameters $\alpha, \beta, \gamma, \delta_{\text {in }}, \delta_{\text {out }}, G_{0}$ of the model to be fixed; all implicit constants in $O(\cdot)$ and $o(\cdot)$ thereafter can depend on those. Also, we do not emphasize dependency on $\varepsilon$ (which will always be an arbitrarily small positive number).

We will explicitly indicate dependencies on other variables using lower indices in $O_{d}(\cdot)$ and $o_{d}(\cdot)$. The notation $f(t)=O_{d}(g(t))$ means that there exists $C_{d}$ such that $|f(t)| \leq$ $C_{d} g(t)$ for all possible values of $t$ and other variables, if any. Here, $C_{d}$ can depend on $d$, parameters of the model and $\varepsilon$, but not on $t$ and other possible variables such as $x$ and $N$. Similarly, the notation $f(t)=o_{d}(g(t))$ when $t \rightarrow \infty$ means that for every $\delta>0$ there exists $t_{d}(\delta)$ (depending on $d, \delta, \varepsilon$ and parameters of the model but not on $t$ and other possible variables) such that $|f(t)| \leq \delta g(t)$ for $t \geq t_{d}(\delta)$.

Let $\bar{c}_{\text {in }}=\frac{1-\gamma}{1+\delta_{\text {in }}(\alpha+\gamma)}$ and $\bar{c}_{\text {out }}=\frac{1-\alpha}{1+\delta_{\text {out }}(\alpha+\gamma)}$.

Our first topic is the in-degree sequence in this model. Obviously, the out-degree sequence has the same structure with exchanging $\alpha \leftrightarrow \gamma$ and $\delta_{\text {in }} \leftrightarrow \delta_{\text {out }}$. Let $n_{\text {in }}(t, d)$ be a number of vertices with in-degree $d$ in a random graph $G \in \mathcal{G}(t)$. There are two special cases for the in-degree sequence that are not interesting. If $\alpha+\beta=0$, then $\bar{c}_{\text {in }}=0$ and every vertex not in $G_{0}$ has in-degree 1 . If $\gamma+\delta_{i n}=0$, then $\bar{c}_{i n}=1$ and every vertex not in $G_{0}$ has in-degree 0 . Otherwise, $0<\bar{c}_{i n}<1$.

Define

$$
\begin{aligned}
\overline{f_{0}} & =\frac{\alpha}{1+\delta_{i n} \bar{c}_{i n}}, \\
\overline{f_{d}} & =C_{i n} \frac{\Gamma\left(d+\delta_{i n}\right)}{\Gamma\left(d+\delta_{i n}+1+\frac{1}{\bar{c}_{i n}}\right)} \text { for } d \geq 1, \\
C_{i n} & =\frac{\Gamma\left(\delta_{i n}+\frac{1}{\bar{c}_{i n}}\right)}{\Gamma\left(1+\delta_{i n}\right)} \frac{1-\bar{c}_{i n}}{\bar{c}_{i n}{ }^{2}} .
\end{aligned}
$$

Then $\overline{f_{d}} \sim C_{i n} d^{-1-\frac{1}{\bar{c}_{i n}}}$ as $d$ grows due to a standard result about the gamma function (e.g., $[1,6.1 .47])$.

It is proven [5] that $n_{i n}(t, d)=\overline{f_{d}} t\left(1+o_{d}(1)\right)$ (with probability tending to 1 as $t \rightarrow \infty)$, i.e., the power law when $d$ is fixed. It is proven [8] that $n_{i n}(t, d)=$ $\overline{f_{d}} t\left(1+O\left(\frac{1}{\sqrt{\log t}}\right)\right.$ ) (again, with probability tending to 1 as $\left.t \rightarrow \infty\right)$ for $d \leq \min \left\{t^{\bar{c}_{i n} / 3}, \frac{t^{1 / 6}}{\log ^{2} t}\right\}$ (in the model of [8] after an appropriate mapping of parameters); for the maximal value of $d$ covered by [8] we have $n_{i n}(t, d) \gg t^{\max \left\{\frac{2-\bar{c}_{i n}}{3}, \frac{5-1 / \bar{c}_{i n}}{6}\right\}} \geq \sqrt{t}$. Our results are valid 
for all possible degrees, given the concentration up to the moment where $n_{i n}(t, d)$ becomes $O\left(\ln ^{2} t\right)$, and we have a much better remainder term for values of $d$ covered by [5] and [8].

Theorem 2.1. Let $\alpha+\gamma>0, \alpha+\beta>0$ and $\gamma+\delta_{\text {in }}>0$. Let $\varepsilon>0$ be arbitrarily small. Let $d=d(t)$. Then

$$
\mathbf{E} n_{i n}(t, d)=\overline{f_{d}} t+O\left((d+1)^{-1+\varepsilon}\right) .
$$

(In compliance with the generic convention above, the implicit constant in $O(\cdot)$ depends on parameters of the model and on $\varepsilon$, but not on $t$ and the function $d(t)$.)

Theorem 2.2. Let $\alpha+\gamma>0, \alpha+\beta>0$ and $\gamma+\delta_{\text {in }}>0$. Let $\varepsilon>0$ be arbitrarily small. Let $d=d(t)$. Then

$$
\left|n_{i n}(t, d)-\overline{f_{d}} t\right| \leq\left(\sqrt{\overline{f_{d}} t}+(d+1)^{-\frac{1}{2}+\varepsilon}\right) \ln t
$$

with probability tending to 1 as $t \rightarrow \infty$.

When $d=o\left(\left(\frac{t}{\ln ^{2} t}\right)^{\frac{\bar{c}_{i n}}{1+\bar{c}_{i n}}}\right)$, that is, equivalent to $\ln ^{2} t=o\left(\overline{f_{d}} t\right)$, Theorem 2.2 implies the equivalence (with probability tending to 1 as $d, t \rightarrow \infty$ )

$$
n_{\text {in }}(t, d) \sim C_{i n} d^{-1-\frac{1}{\bar{c}_{\text {in }}}} t
$$

When $t^{\frac{\bar{c}_{i n}}{1+\bar{c}_{i n}}}=o(d)$, Theorem 2.1 implies $\mathbf{E} n_{i n}(t, d)=o(1)$; since $n_{i n}(t, d)$ is an integer number by definition, $n_{i n}(t, d)=0$ (again, with probability tending to 1 as $d, t \rightarrow \infty$ ). Thus, we have an almost entire picture of what happens to $n_{i n}(t, d)$.

Our second topic concerns the expected number of edges (or probability of an edge) between two given vertices. Suppose that we know their in- and out-degrees, but nothing else. For a fixed vertex, in-degree and out-degree are essentially independent due to the construction (although they both tend to grow with age). Thus, we use only out-degree $d_{1}$ of the potential source and in-degree $d_{2}$ of the potential target. Theorems 2.1 and 2.2 give the total number of vertices with in-degree $d_{2}$ and the total number of vertices with out-degree $d_{1}$. Thus, we estimate the total number of edges between vertices of given degrees. For this topic, we consider $d_{1}$ and $d_{2}$ to be fixed, not growing with $t$, to simplify calculations somewhat.

More precisely, we define the function $X\left(G, d_{1}, d_{2}\right)$ of a graph $G$ as the total number of edges with the following property: the out-degree of the source vertex is $d_{1} \geq 1$, the in-degree of the target vertex is $d_{2} \geq 1$, source and target vertices are different (i.e., for $d_{1}=d_{2}$ we do not count loops). When $G$ is a random graph from $\mathcal{G}(t)$, $X\left(G, d_{1}, d_{2}\right)$ yields a random variable; denote it as $X\left(t, d_{1}, d_{2}\right)$. The average number of edges between two vertices of degrees $d_{1}$ and $d_{2}$ in a random graph in the model is $\frac{X\left(t, d_{1}, d_{2}\right)}{n_{\text {out }}\left(t, d_{1}\right) n_{\text {in }}\left(t, d_{2}\right)}$. 
Define

$$
\begin{aligned}
& \kappa\left(c_{1}, c_{2}, r, x\right) \\
& \quad=\int_{0}^{x} z^{c_{1}-1} d z \int_{0}^{\infty} \tau^{c_{1} r+c_{2}-1} e^{-\tau-z \tau^{r}} d \tau=x^{c_{1}} \int_{0}^{1} z^{c_{1}-1} d z \int_{0}^{\infty} \tau^{c_{1} r+c_{2}-1} e^{-\tau-x z \tau^{r}} d \tau
\end{aligned}
$$

for $c_{1}>0, c_{2}>0, r>0, x \geq 0$.

As a function of $x$, it monotonically increases from 0 to $\Gamma\left(c_{1}\right) \Gamma\left(c_{2}\right)$ as $x$ grows from 0 to $\infty$ (the limit at $\infty$ immediately follows from Lemma 5.4 following). The asymptotic behavior when $x \rightarrow 0$ is given by $\kappa\left(c_{1}, c_{2}, r, x\right)=\left(\frac{\Gamma\left(c_{1} r+c_{2}\right)}{c_{1}}+O(x)\right) x^{c_{1}}$.

Note: in the special case $r=1$, the inner integral can be calculated and $\kappa$ becomes the incomplete beta-function: $\kappa\left(c_{1}, c_{2}, 1, x\right)=\Gamma\left(c_{1}+c_{2}\right) \mathrm{B}\left(\frac{x}{1+x} ; c_{1}, c_{2}\right)$. We won't use this fact, but it can be useful to conceive the function $\kappa$.

Theorem 2.3. Let $\alpha>0, \beta>0, \gamma>0, \delta_{\text {in }}>0, \delta_{\text {out }}>0, d_{1} \geq 2, d_{2} \geq 2$. Then, $\mathbf{E} X\left(t, d_{1}, d_{2}\right)=c_{X}\left(d_{1}, d_{2}\right) t+O_{d_{1}, d_{2}}(1)$, where

$$
c_{X}\left(d_{1}, d_{2}\right)=c_{X 1}\left(d_{1}, d_{2}\right)+c_{X 2}\left(d_{1}, d_{2}\right)+c_{X 3}\left(d_{1}, d_{2}\right),
$$

and

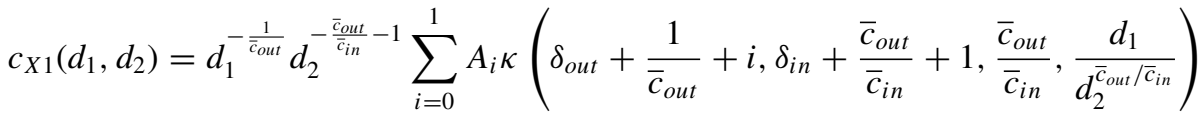

$$
\begin{aligned}
& \times\left(1+O\left(\frac{1}{d_{1}}+\frac{1}{d_{2}}\right)\right), \\
& A_{0}=\frac{\gamma^{2}}{\left(1+\delta_{\text {out }}(\alpha+\gamma)\right) \bar{c}_{\text {in }} \bar{c}_{\text {out }} \Gamma\left(\delta_{\text {out }}\right) \Gamma\left(1+\delta_{\text {in }}\right)}, \\
& A_{1}=\frac{\alpha \gamma}{\left(1+\delta_{\text {out }}(\alpha+\gamma)\right) \bar{c}_{\text {in }} \bar{c}_{\text {out }} \Gamma\left(1+\delta_{\text {out }}\right) \Gamma\left(1+\delta_{\text {in }}\right)}, \\
& c_{X 2}\left(d_{1}, d_{2}\right)=d_{1}^{-\frac{\bar{c}_{\text {in }}}{\bar{c}_{\text {out }}}-1} d_{2}^{-\frac{1}{\bar{c}_{\text {in }}}} \sum_{i=0}^{1} B_{i} \kappa\left(\delta_{\text {in }}+\frac{1}{\bar{c}_{\text {in }}}+i, \delta_{\text {out }}+\frac{\bar{c}_{\text {in }}}{\bar{c}_{\text {out }}}+1, \frac{\bar{c}_{\text {in }}}{\bar{c}_{\text {out }}}, \frac{d_{2}}{d_{1}^{\bar{c}_{\text {in }} / \bar{c}_{\text {out }}}}\right) \\
& \times\left(1+O\left(\frac{1}{d_{1}}+\frac{1}{d_{2}}\right)\right), \\
& B_{0}=\frac{\alpha^{2}}{\left(1+\delta_{\text {in }}(\alpha+\gamma)\right) \bar{c}_{\text {in }} \bar{c}_{\text {out }} \Gamma\left(1+\delta_{\text {out }}\right) \Gamma\left(\delta_{\text {in }}\right)}, \\
& B_{1}=\frac{\alpha \gamma}{\left(1+\delta_{\text {in }}(\alpha+\gamma)\right) \bar{c}_{\text {in }} \bar{c}_{\text {out }} \Gamma\left(1+\delta_{\text {out }}\right) \Gamma\left(1+\delta_{\text {in }}\right)} .
\end{aligned}
$$


If $\bar{c}_{\text {in }}+\bar{c}_{\text {out }} \neq 1$, then

$$
\begin{aligned}
c_{X 3}\left(d_{1}, d_{2}\right)= & d_{1}^{-\frac{1}{\bar{c}_{\text {out }}}} d_{2}^{-\frac{1}{\bar{c}_{\text {in }}}} \sum_{i=0}^{1} \sum_{j=0}^{1} \frac{C_{\text {ij }}}{1-\bar{c}_{\text {in }}-\bar{c}_{\text {out }}} \\
& \times\left(d_{2}^{\frac{1-\bar{c}_{\text {in }}-\overline{-}_{\text {out }}}{\bar{c}_{\text {in }}}} \kappa\left(\delta_{\text {out }}+\frac{1}{\bar{c}_{\text {out }}}+i, \delta_{\text {in }}+\frac{\bar{c}_{\text {out }}}{\bar{c}_{\text {in }}}+1+j, \frac{\bar{c}_{\text {out }}}{\bar{c}_{\text {in }}}, \frac{d_{1}}{d_{2}^{\bar{c}_{\text {out }} / \bar{c}_{\text {in }}}}\right)\right. \\
& \times\left(1+O\left(\frac{1}{d_{1}}+\frac{1}{d_{2}}\right)\right)+d_{1}^{\frac{1-\bar{c}_{\text {in }}-\overline{\bar{c}}_{\text {out }}}{\bar{c}_{\text {out }}}} \\
& \times \kappa\left(\delta_{\text {in }}+\frac{1}{\bar{c}_{\text {in }}}+j, \delta_{\text {out }}+\frac{\bar{c}_{\text {in }}}{\bar{c}_{\text {out }}}+1+i, \frac{\bar{c}_{\text {in }}}{\bar{c}_{\text {out }}}, \frac{d_{2}}{\left.d_{1}^{\bar{c}_{\text {in }} / \bar{c}_{\text {out }}}\right)}\right. \\
& \times\left(1+O\left(\frac{1}{d_{1}}+\frac{1}{d_{2}}\right)\right)-\Gamma\left(\delta_{\text {out }}+\frac{1}{\bar{c}_{\text {out }}}+i\right) \Gamma\left(\delta_{\text {in }}+\frac{1}{\bar{c}_{\text {in }}}+j\right) \\
& \left.+O\left(\frac{1}{d_{1}}+\frac{1}{d_{2}}\right)\right),
\end{aligned}
$$

$$
\begin{aligned}
& C_{00}=\frac{\beta \alpha \gamma}{\left(1+\delta_{\text {in }}(\alpha+\gamma)\right)\left(1+\delta_{\text {out }}(\alpha+\gamma)\right) \bar{c}_{\text {in }} \bar{c}_{\text {out }} \Gamma\left(\delta_{\text {out }}\right) \Gamma\left(\delta_{\text {in }}\right)}, \\
& C_{01}=\frac{\beta \gamma^{2}}{\left(1+\delta_{\text {in }}(\alpha+\gamma)\right)\left(1+\delta_{\text {out }}(\alpha+\gamma)\right) \bar{c}_{\text {in }} \bar{c}_{\text {out }} \Gamma\left(\delta_{\text {out }}\right) \Gamma\left(\delta_{\text {in }}+1\right)}, \\
& C_{10}=\frac{\beta \alpha^{2}}{\left(1+\delta_{\text {in }}(\alpha+\gamma)\right)\left(1+\delta_{\text {out }}(\alpha+\gamma)\right) \bar{c}_{\text {in }} \bar{c}_{\text {out }} \Gamma\left(\delta_{\text {out }}+1\right) \Gamma\left(\delta_{\text {in }}\right)}, \\
& C_{11}=\frac{\beta \alpha \gamma}{\left(1+\delta_{\text {in }}(\alpha+\gamma)\right)\left(1+\delta_{\text {out }}(\alpha+\gamma)\right) \bar{c}_{\text {in }} \bar{c}_{\text {out }} \Gamma\left(\delta_{\text {out }}+1\right) \Gamma\left(\delta_{\text {in }}+1\right)} . \\
& \text { If } \bar{c}_{\text {in }}+\bar{c}_{\text {out }}=1, \text { then }
\end{aligned}
$$

$$
\begin{aligned}
c_{X 3}\left(d_{1}, d_{2}\right) & d_{1}^{-\frac{1}{\bar{c}_{\text {out }}}} d_{2}^{-\frac{1}{\bar{c}_{\text {in }}}} \sum_{i=0}^{1} \sum_{j=0}^{1} C_{i j}\left(\frac{1}{\bar{c}_{\text {in }}} \kappa\left(\delta_{\text {out }}+\frac{1}{\bar{c}_{\text {out }}}+i, \delta_{\text {in }}+\frac{1}{\bar{c}_{\text {in }}}+j, \frac{\bar{c}_{\text {out }}}{\bar{c}_{\text {in }}}, \frac{d_{1}}{\bar{d}_{2}^{\bar{c}_{\text {out }} / \bar{c}_{\text {in }}}}\right) \ln d_{2}\right. \\
& -\frac{1}{\bar{c}_{\text {in }}} \frac{\partial \kappa}{\partial c_{2}}\left(\delta_{\text {out }}+\frac{1}{\bar{c}_{\text {out }}}+i, \delta_{\text {in }}+\frac{1}{\bar{c}_{\text {in }}}+j, \frac{\bar{c}_{\text {out }}}{\bar{c}_{\text {in }}}, \frac{d_{1}}{d_{2}^{\bar{c}_{\text {out }} / \bar{c}_{\text {in }}}}\right) \\
& +\frac{1}{\bar{c}_{\text {out }}} \kappa\left(\delta_{\text {in }}+\frac{1}{\bar{c}_{\text {in }}}+j, \delta_{\text {out }}+\frac{1}{\bar{c}_{\text {out }}}+i, \frac{\bar{c}_{\text {in }}}{\bar{c}_{\text {out }}}, \frac{d_{2}}{d_{1}^{\bar{c}_{\text {in }} / \bar{c}_{\text {out }}}}\right) \ln d_{1} \\
& \left.-\frac{1}{\bar{c}_{\text {out }}} \frac{\partial \kappa}{\partial c_{2}}\left(\delta_{\text {in }}+\frac{1}{\bar{c}_{\text {in }}}+j, \delta_{\text {out }}+\frac{1}{\bar{c}_{\text {out }}}+i, \frac{\bar{c}_{\text {in }}}{\bar{c}_{\text {out }}}, \frac{d_{2}}{d_{1}^{\bar{c}_{\text {in }} / \bar{c}_{\text {out }}}}\right)+O\left(\frac{\ln d_{2}}{d_{1}}+\frac{\ln d_{1}}{d_{2}}\right)\right)
\end{aligned}
$$

(where $C_{i j}$ are the same as in the previous case). 
If $d_{1}$ and $d_{2}$ both grow to infinity such that $d_{1}^{\bar{c}_{\text {in }}} / d_{2}^{\bar{c}_{\text {out }}} \rightarrow 0$, then

$$
\begin{aligned}
& c_{X}\left(d_{1}, d_{2}\right) \sim \begin{cases}D_{-} d_{1}^{-\frac{\bar{c}_{\text {in }}+\bar{c}_{\text {out }}}{\bar{c}_{\text {out }}}} d_{2}^{-\frac{1}{\bar{c}_{\text {in }}}}, & \bar{c}_{\text {in }}+\bar{c}_{\text {out }}<1 \\
D_{0} d_{1}^{-\frac{1}{\bar{c}_{\text {out }}}} d_{2}^{-\frac{1}{\bar{c}_{\text {in }}}} \ln d_{1}, & \bar{c}_{\text {in }}+\bar{c}_{\text {out }}=1 \\
D_{+} d_{1}^{-\frac{1}{\bar{c}_{\text {out }}}} d_{2}^{-\frac{1}{\bar{c}_{\text {in }}}}, & \bar{c}_{\text {in }}+\bar{c}_{\text {out }}>1,\end{cases} \\
& D_{-}=\left(B_{0} \Gamma\left(\delta_{\text {in }}+\frac{1}{\bar{c}_{\text {in }}}\right)+B_{1} \Gamma\left(\delta_{\text {in }}+\frac{1}{\bar{c}_{\text {in }}}+1\right)\right) \Gamma\left(\delta_{\text {out }}+\frac{\bar{c}_{\text {in }}}{\bar{c}_{\text {out }}}+1\right) \\
& +\sum_{i=0}^{1} \sum_{j=0}^{1} \frac{C_{i j}}{1-\bar{c}_{\text {in }}-\bar{c}_{\text {out }}} \Gamma\left(\delta_{\text {in }}+\frac{1}{\bar{c}_{\text {in }}}+j\right) \Gamma\left(\delta_{\text {out }}+\frac{\bar{c}_{\text {in }}}{\bar{c}_{\text {out }}}+1+i\right) ; \\
& D_{0}=\sum_{i=0}^{1} \sum_{j=0}^{1} \frac{C_{\text {ij }}}{\bar{c}_{\text {out }}} \Gamma\left(\delta_{\text {out }}+\frac{1}{\bar{c}_{\text {out }}}+i\right) \Gamma\left(\delta_{\text {in }}+\frac{1}{\bar{c}_{\text {in }}}+j\right) \text {; } \\
& D_{+}=\sum_{i=0}^{1} \sum_{j=0}^{1} \frac{C_{i j}}{\bar{c}_{\text {in }}+\bar{c}_{\text {out }}-1} \Gamma\left(\delta_{\text {out }}+\frac{1}{\bar{c}_{\text {out }}}+i\right) \Gamma\left(\delta_{\text {in }}+\frac{1}{\bar{c}_{\text {in }}}+j\right) .
\end{aligned}
$$

If $d_{1}$ and $d_{2}$ both grow to infinity such that $d_{1}^{\bar{c}_{\text {in }}} / d_{2}^{\bar{c}_{\text {out }}} \rightarrow \infty$, then

$$
\begin{gathered}
c_{X}\left(d_{1}, d_{2}\right) \sim \begin{cases}D_{-}^{\prime} d_{1}^{-\frac{1}{\bar{c}_{\text {out }}}} d_{2}^{-\frac{\bar{c}_{\text {in }}+\bar{c}_{\text {out }}}{\bar{c}_{\text {in }}}}, & \bar{c}_{\text {in }}+\bar{c}_{\text {out }}<1 \\
D_{0}^{\prime} d_{1}^{-\frac{1}{\bar{c}_{\text {out }}}} d_{2}^{-\frac{1}{\bar{c}_{\text {in }}}} \ln d_{2}, & \bar{c}_{\text {in }}+\bar{c}_{\text {out }}=1 \\
D_{+} d_{1}^{-\frac{1}{\bar{c}_{\text {out }}}} d_{2}^{-\frac{1}{\bar{c}_{\text {in }}}}, & \bar{c}_{\text {in }}+\bar{c}_{\text {out }}>1,\end{cases} \\
D_{-}^{\prime}=\left(A_{0} \Gamma\left(\delta_{\text {out }}+\frac{1}{\bar{c}_{\text {out }}}\right)+A_{1} \Gamma\left(\delta_{\text {out }}+\frac{1}{\bar{c}_{\text {out }}}+1\right)\right) \Gamma\left(\delta_{\text {in }}+\frac{\bar{c}_{\text {out }}}{\bar{c}_{\text {in }}}+1\right) \\
+\sum_{i=0}^{1} \sum_{j=0}^{1} \frac{C_{i j}}{1-\bar{c}_{\text {in }}-\bar{c}_{\text {out }}} \Gamma\left(\delta_{\text {in }}+\frac{\bar{c}_{\text {out }}}{\bar{c}_{\text {in }}}+1+j\right) \Gamma\left(\delta_{\text {out }}+\frac{1}{\bar{c}_{\text {out }}}+i\right) ; \\
D_{0}^{\prime}=\sum_{i=0}^{1} \sum_{j=0}^{1} \frac{C_{i j}}{\bar{c}_{\text {in }}} \Gamma\left(\delta_{\text {out }}+\frac{1}{\bar{c}_{\text {out }}}+i\right) \Gamma\left(\delta_{\text {in }}+\frac{1}{\bar{c}_{\text {in }}}+j\right) .
\end{gathered}
$$

Note: one can see from the proof that $c_{X 1}, c_{X 2}, c_{X 3}$ have their own physical sense: they give the fraction of edges produced by $(\ddagger),(\dagger)$, and $(\dagger \dagger)$, respectively (relative to $t$, the total number of edges).

Theorem 2.4. Let $d_{1}$ and $d_{2}$ be fixed. In conditions of the previous theorem

$$
\left|X\left(t, d_{1}, d_{2}\right)-\mathbf{E} X\left(t, d_{1}, d_{2}\right)\right|<\sqrt{t} \ln t
$$

with probability tending to 1 as $t \rightarrow \infty$. 
The average value of the number of edges from a vertex with out-degree $d_{1}$ to a vertex with in-degree $d_{2}$ is $\frac{X\left(t, d_{1}, d_{2}\right)}{n_{\text {out }}\left(t, d_{1}\right) n_{i n}\left(t, d_{2}\right)}$. Since $n_{\text {in }}(t, d)$ and $X\left(t, d_{1}, d_{2}\right)$ are tightly concentrated around their expectations, the main term of the ratio is given by Theorems 2.1 and 2.3. In particular, if $\bar{c}_{\text {in }}+\bar{c}_{\text {out }}>1$, the average number of edges is proportional to $\frac{d_{1} d_{2}}{t}$, and if $\bar{c}_{\text {in }}+\bar{c}_{\text {out }}<1$, it grows somehow between $\frac{1}{t} d_{1}^{\frac{1-\bar{c}_{\text {in }}}{\bar{c}_{\text {out }}}} d_{2}$ and $\frac{1}{t} d_{1} d_{2}^{\frac{1-\bar{c}_{\text {out }}}{\bar{c}_{\text {in }}}}$ (tending to the first expression when $d_{1}$ is small compared to $d_{2}$ and to the second one when $d_{1}$ is large; Theorem 2.3 describes intermediate cases in detail).

\section{EXPECTED NUMBER OF VERTICES WITH THE GIVEN DEGREE}

We will use some additional notation for all the proofs following.

For a given graph $G$, let \# $G$ be the number of vertices in $G$ and $n_{i n}(G, d)$ be the number of vertices in $G$ with in-degree $d$. For a random graph $G \in \mathcal{G}(t)$, \#G is a random variable defined on $\mathcal{G}(t)$ and $n_{i n}(G, d)$ becomes the random variable $n_{\text {in }}(t, d)$.

For a property $P$, we denote

$$
[P]= \begin{cases}1, & P \text { holds } \\ 0, & \text { otherwise. }\end{cases}
$$

Proof of Theorem 2.1 Let $n_{0}=\# G_{0}, T=t-t_{0}, A_{\text {in }}=t_{0}+\delta_{\text {in }} n_{0}, 0 \leq N \leq T$ if $\beta>0$ and

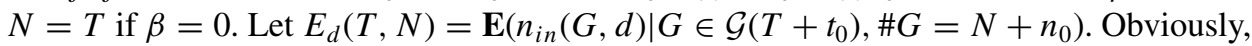
in the probability space $\mathcal{G}\left(T+t_{0}\right)$,

$\mathbf{E} n_{i n}\left(T+t_{0}, d\right)=\sum_{N=0}^{T} E_{d}(T, N) \operatorname{Pr}\left(\# G=N+n_{0}\right)=\sum_{N=0}^{T} E_{d}(T, N)(\alpha+\gamma)^{N} \beta^{T-N}\left(\begin{array}{c}T \\ N\end{array}\right)$.

For $x \in[0,1]$, let $c_{i n}(x)=\frac{1-\frac{\gamma}{\alpha+\gamma} x}{1+\delta_{\text {in }} x} \in[0,1]$ and $c_{\text {out }}(x)=\frac{1-\frac{\alpha}{\alpha+\gamma} x}{1+\delta_{\text {out }} x} \in[0,1]$. Note that $\bar{c}_{i n}=c_{\text {in }}(\alpha+\gamma), \bar{c}_{\text {out }}=c_{\text {out }}(\alpha+\gamma)$. Let $p_{\text {out }, 0}=p_{\text {in }, 1}=\frac{\gamma}{\alpha+\gamma}, p_{\text {out }, 1}=p_{\text {in }, 0}=\frac{\alpha}{\alpha+\gamma}$.

Lemma 3.1. Let $\alpha+\gamma>0, \alpha+\beta>0, \gamma+\delta_{\text {in }}>0, \varepsilon>0$. For $T \geq 1, d \geq 0$, $0 \leq N \leq T$ if $\beta>0$ and $N=T$ if $\beta=0$,

$$
E_{d}(T, N)=T f_{d}\left(\frac{N}{T}\right)+O\left(\frac{1}{1+\left(d+\delta_{i n}+1\right)^{1-\varepsilon} N^{2} / T^{2}}\right),
$$

where

$$
\begin{gathered}
f_{0}(x)=\frac{a x}{1+c_{i n}(x) \delta_{i n}}, \quad a=\frac{\alpha}{\alpha+\gamma}, \\
f_{d}(x)=\frac{1-c_{i n}(x)}{\left(1+c_{i n}(x) \delta_{i n}\right)\left(1+c_{i n}(x)\left(\delta_{i n}+1\right)\right)} \prod_{i=2}^{d} \frac{c_{i n}(x)\left(\delta_{i n}+i-1\right)}{1+c_{i n}(x)\left(\delta_{i n}+i\right)} \text { for } d \geq 1 .
\end{gathered}
$$

If $\delta_{\text {in }}>0$ and either $\alpha>0$ or $x<1$, then

$$
f_{d}(x)=x \sum_{i=0}^{1}[d \geq i] p_{i n, i} \frac{\Gamma\left(i+\delta_{i n}+\frac{1}{c_{i n}}(x)\right)}{c_{i n}(x) \Gamma\left(i+\delta_{i n}\right)} \frac{\Gamma\left(d+\delta_{i n}\right)}{\Gamma\left(d+1+\delta_{i n}+\frac{1}{c_{i n}(x)}\right)} .
$$


Proof. Without loss of generality assume $\varepsilon<1$.

First, derive a recurrent equation for $E_{d}(T, N)$. Let $d_{i n}(G, i)$ denote in-degree of a vertex $i$ in a graph $G$.

$$
\begin{aligned}
E_{d}(T+1, N) & =\sum_{i=1}^{N+n_{0}} \operatorname{Pr}\left(d_{i n}\left(G_{T+1}, i\right)=d \mid G_{T+1} \in \mathcal{G}\left(T+t_{0}+1\right), \# G_{T+1}=N+n_{0}\right) \\
& =\sum_{i=1}^{N+n_{0}} \frac{\operatorname{Pr}\left(d_{i n}\left(G_{T+1}, i\right)=d, \# G_{T+1}=N+n_{0} \mid G_{T+1} \in \mathcal{G}\left(T+t_{0}+1\right)\right)}{\operatorname{Pr}\left(\# G_{T+1}=N+n_{0} \mid G_{T+1} \in \mathcal{G}\left(T+t_{0}+1\right)\right)}
\end{aligned}
$$

The denominator is easy to calculate: $\operatorname{Pr}\left(\# G_{T+1}=N+n_{0}\right)=(\alpha+\gamma)^{N} \beta^{T+1-N}\left(\begin{array}{c}T+1 \\ N\end{array}\right)$.

Let $G_{T+1}$ be a random graph from $\mathcal{G}\left(T+t_{0}+1\right)$ with $N+n_{0}$ vertices. The numerator can be expressed as a sum $\operatorname{Pr}(\ldots, \dagger)+\operatorname{Pr}(\ldots, \ddagger)+\operatorname{Pr}(\ldots, \dagger \dagger)$ of probabilities with the additional condition that $G_{T+1}$ is constructed from $G_{T} \in \mathcal{G}\left(T+t_{0}\right)$ using $(\dagger),(\ddagger),(\dagger \dagger)$,

respectively.

- If $G_{T+1}$ is constructed from $G_{T} \in \mathcal{G}\left(T+t_{0}\right)$ using (†), then $N>0, G_{T}$ has $N+n_{0}-1$ vertices. The last vertex in $G_{T+1}$ has in-degree 0 . For $i \in G_{T}$,

$$
\begin{aligned}
\operatorname{Pr}\left(d_{i n}\left(G_{T+1}, i\right)=\right. & \left.d, \# G_{T+1}=N+n_{0}, \dagger\right)=\alpha \operatorname{Pr}\left(d_{i n}\left(G_{T}, i\right)=d, \# G_{T}=N+n_{0}-1\right) \\
& \times\left(1-\frac{d+\delta_{i n}}{T+\delta_{i n} N+A_{i n}-\delta_{i n}}\right) \\
& +\alpha \operatorname{Pr}\left(d_{i n}\left(G_{T}, i\right)=d-1, \# G_{T}=N+n_{0}-1\right) \\
& \times \frac{d-1+\delta_{i n}}{T+\delta_{i n} N+A_{\text {in }}-\delta_{i n}} .
\end{aligned}
$$

Note that

$$
\frac{\operatorname{Pr}\left(\# G_{T}=N+n_{0}-1\right)}{\operatorname{Pr}\left(\# G_{T+1}=N+n_{0}\right)}=\frac{(\alpha+\gamma)^{N-1} \beta^{T+1-N}\left(\begin{array}{c}
T \\
N-1
\end{array}\right)}{(\alpha+\gamma)^{N} \beta^{T+1-N}\left(\begin{array}{c}
T+1 \\
N
\end{array}\right)}=\frac{N}{(\alpha+\gamma)(T+1)} .
$$

- If $G_{T+1}$ is constructed from $G_{T} \in \mathcal{G}\left(T+t_{0}\right)$ using (††), then $N<T+1, G_{T}$ has $N+n_{0}$ vertices. For each of them

$$
\begin{aligned}
\operatorname{Pr}\left(d_{i n}\left(G_{T+1}, i\right)=d,\right. & \left.\# G_{T+1}=N+n_{0}, \dagger \dagger\right) \\
= & \beta \operatorname{Pr}\left(d_{i n}\left(G_{T}, i\right)=d, \# G_{T}=N+n_{0}\right)\left(1-\frac{d+\delta_{i n}}{T+\delta_{i n} N+A_{i n}}\right) \\
& +\beta \operatorname{Pr}\left(d_{i n}\left(G_{T}, i\right)=d-1, \# G_{T}=N+n_{0}\right) \frac{d-1+\delta_{i n}}{T+\delta_{i n} N+A_{i n}} .
\end{aligned}
$$


Note that

$$
\frac{\operatorname{Pr}\left(\# G_{T}=N+n_{0}\right)}{\operatorname{Pr}\left(\# G_{T+1}=N+n_{0}\right)}=\frac{(\alpha+\gamma)^{N} \beta^{T-N}\left(\begin{array}{c}
T \\
N
\end{array}\right)}{(\alpha+\gamma)^{N} \beta^{T+1-N}\left(\begin{array}{c}
T+1 \\
N
\end{array}\right)}=\frac{T+1-N}{\beta(T+1)} .
$$

- If $G_{T+1}$ is constructed from $G_{T} \in \mathcal{G}\left(T+t_{0}\right)$ using ( $\left.\ddagger\right)$, then $N>0, G_{T}$ has $N+n_{0}-1$ vertices. The last vertex in $G_{T+1}$ has in-degree 1. For $i \in G_{T}$,

$$
\operatorname{Pr}\left(d_{i n}\left(G_{T+1}, i\right)=d, \# G_{T+1}=N+n_{0}, \ddagger\right)=\gamma \operatorname{Pr}\left(d_{i n}\left(G_{T}, i\right)=d, \# G_{T}=N+n_{0}-1\right) .
$$

Thus,

$$
\begin{aligned}
E_{d}(T+1, N)= & {[N>0, d=0] \alpha \frac{N}{(\alpha+\gamma)(T+1)} } \\
& +[N>0] \alpha E_{d}(T, N-1)\left(1-\frac{d+\delta_{i n}}{T+\delta_{i n} N+A_{i n}-\delta_{i n}}\right) \frac{N}{(\alpha+\gamma)(T+1)} \\
& \left.+[N>0] \alpha E_{d-1}(T, N-1) \frac{d-1+\delta_{i n}}{T+\delta_{i n} N+A_{\text {in }}-\delta_{i n}} \frac{N+\gamma)(T+1)}{\left(\alpha+\delta_{i n}\right.}\right) \frac{T+1-N}{\beta(T+1)} \\
& +[N<T+1] \beta E_{d}(T, N)\left(1-\frac{d+\delta_{i n} N+A_{i n}}{T+\delta_{i n}} \frac{T+1-N}{\beta(T+1)}\right. \\
& +[N<T+1] \beta E_{d-1}(T, N) \frac{d-1+\delta_{i n} N+A_{i n}}{T+\delta_{i n}} \\
& +[N>0, d=1] \gamma \frac{N}{(\alpha+\gamma)(T+1)} \\
& +[N>0] \gamma E_{d}(T, N-1) \frac{N}{(\alpha+\gamma)(T+1)}
\end{aligned}
$$

We need to establish some properties of $f_{d}(x)$. A straightforward calculation shows that two definitions of $f_{d}(x)$ from the statement of the lemma are indeed equivalent (when the second one is defined) and that $f_{d}(x)$ satisfy to the following recurrent equation:

$$
\begin{aligned}
& \left(\frac{1-x+a x}{1+\delta_{i n} x}\left(d+\delta_{i n}\right)+1\right) f_{d}(x)=\frac{1-x+a x}{1+\delta_{i n} x}\left(d+\delta_{i n}-1\right) f_{d-1}(x) \\
& \quad+x([d=0] a+[d=1](1-a)),
\end{aligned}
$$

assuming $f_{-1}(x)=0$. It is obvious from the first definition of $f_{d}(x)$ that $f_{d}(x)$ is analytical for $x \in[0,1]$ and any fixed $d$. Apply generalized Bernoulli inequality $(1+z)^{r} \geq 1+z r$ 
(valid for $z \geq-1, r \geq 1$ or $r \leq 0$ ) to $z=\frac{1}{\delta_{i n}+i+\frac{1}{c_{i n}(x)+\frac{\varepsilon}{2}}}, r=-1-\frac{1}{c_{i n}(x)+\frac{\varepsilon}{2}}$ :

$$
\begin{aligned}
& \prod_{i=2}^{d} \frac{c_{i n}(x)\left(\delta_{i n}+i-1\right)}{1+c_{i n}(x)\left(\delta_{i n}+i\right)} \leq \prod_{i=2}^{d} \frac{\delta_{i n}+i-1}{\delta_{i n}+i+\frac{1}{c_{i n}(x)+\frac{\varepsilon}{2}}} \leq \prod_{i=2}^{d}\left(1+\frac{1}{\delta_{i n}+i+\frac{1}{c_{i n}(x)+\frac{\varepsilon}{2}}}\right)^{-1-\frac{1}{c_{i n}(x)+\frac{\varepsilon}{2}}} \\
& \quad=\left(\frac{d+\delta_{i n}+1+\frac{1}{c_{i n}(x)+\frac{\varepsilon}{2}}}{\delta_{i n}+2+\frac{1}{c_{i n}(x)+\frac{\varepsilon}{2}}}\right)^{-1-\frac{1}{c_{i n}(x)+\frac{\varepsilon}{2}}} \leq\left(\delta_{i n}+2+\frac{2}{\varepsilon}\right)^{1+\frac{2}{\varepsilon}} \\
& \left(d+\delta_{i n}+1\right)^{-1-\frac{1}{c_{i n}(x)+\frac{\varepsilon}{2}}}=O\left(\left(d+\delta_{i n}+1\right)^{-1-\frac{1}{c_{i n}(x)+\frac{\varepsilon}{2}}}\right),
\end{aligned}
$$

where the implied constant does not depend on $x$. Furthermore, for $d \geq 3$ and $c_{i n}(x) \neq 0$, we can separate two first factors of the left-hand side in (3.3), each of them is $O\left(c_{i n}(x)\right)$, and apply the same process to the product of other factors. This gives $\prod_{i=2}^{d} \frac{c_{i n}(x)\left(\delta_{i n}+i-1\right)}{1+c_{i n}(x)\left(\delta_{i n}+i\right)}=$ $O\left(c_{i n}(x)^{2}\left(d+\delta_{i n}+1\right)^{-1-\frac{1}{c_{i n}(x)+\frac{\varepsilon}{2}}}\right)$, so

$$
\begin{aligned}
& \frac{d}{d x} \prod_{i=2}^{d} \frac{c_{i n}(x)\left(\delta_{i n}+i-1\right)}{1+c_{i n}(x)\left(\delta_{i n}+i\right)} \\
& =c_{i n}^{\prime}(x)\left(\prod_{i=2}^{d} \frac{c_{i n}(x)\left(\delta_{i n}+i-1\right)}{1+c_{i n}(x)\left(\delta_{i n}+i\right)}\right)\left(\sum_{i=2}^{d} \frac{1}{c_{i n}(x)\left(1+c_{i n}(x)\left(\delta_{i n}+i\right)\right)}\right) \\
& =O\left(\left(d+\delta_{i n}+1\right)^{-1-\frac{1}{c_{i n}(x)+\frac{\varepsilon}{2}}} \sum_{i=2}^{d} \frac{c_{i n}(x)}{1+c_{i n}(x)\left(\delta_{i n}+i\right)}\right) \\
& =O\left(\left(d+\delta_{i n}+1\right)^{-1-\frac{1}{c_{i n}(x)+\frac{\varepsilon}{2}}} \sum_{i=2}^{d} \frac{1}{1+\delta_{i n}+i}\right) \\
& =O\left(\left(d+\delta_{i n}+1\right)^{-1-\frac{1}{c_{i n}(x)+\frac{\varepsilon}{2}}} \ln d\right)=O\left(\left(d+\delta_{i n}+1\right)^{-1-\frac{1}{c_{i n}(x)+\varepsilon}}\right)
\end{aligned}
$$

where the implied constant again does not depend on $x$; since $f_{d}^{\prime}(x)$ is a linear combination of the left-hand sides of (3.3) and (3.4) with coefficients that are $O(1)$ and $f_{d}^{\prime}(x)$ is continuous, we have

$$
f_{d}^{\prime}(x)=O\left(\left(d+\delta_{i n}+1\right)^{-1-\frac{1}{c_{i n}(x)+\varepsilon}}\right)
$$

for any $x \in[0,1]$ (even in the point $x=1$, where $c_{i n}(x)$ may be zero) and any $d$ (because obviously, $\left.f_{0}^{\prime}(x)=O(1), f_{1}^{\prime}(x)=O(1), f_{2}^{\prime}(x)=O(1)\right)$. Similarly,

$$
f_{d}^{\prime \prime}(x)=O\left(\left(d+\delta_{i n}+1\right)^{-1-\frac{1}{c_{i n}(x)+\frac{\varepsilon}{2}}} \ln ^{2}(d+2)\right)=O\left(\left(d+\delta_{i n}+1\right)^{-1-\frac{1}{c_{i n}(x)+\varepsilon}}\right) .
$$


For this theorem, it is more convenient to use weaker bounds that do not depend on $x$ : $f_{d}(x)=O\left((d+1)^{-2+\varepsilon}\right), f_{d}^{\prime}(x)=O\left((d+1)^{-2+\varepsilon}\right), f_{d}^{\prime \prime}(x)=O\left((d+1)^{-2+\varepsilon}\right)$. A stronger bound (3.3) would not improve the final bound; we will need it for the next theorem, though.

The case $d=\delta_{i n}=0$ is special. In this case, (3.1) becomes

$$
E_{0}(T+1, N)=\frac{a N}{T+1}+[N>0] E_{0}(T, N-1) \frac{N}{T+1}+[N<T+1] E_{0}(T, N) \frac{T+1-N}{T+1} .
$$

The solution is $E_{0}(T, N)=a N+E_{0}(0,0)$, it satisfies the condition of the lemma.

We use induction by $T$ and, for fixed $T$, by $d$ to prove the formula

$$
\left|E_{d}(T, N)-T f_{d}\left(\frac{N}{T}\right)\right| \leq C\left(d, \frac{N}{T}\right), \quad 0 \leq N \leq T
$$

where $C(d, x)=\frac{C_{0}}{1+\left(d+\delta_{i n}+1\right)^{1-\varepsilon} x^{2}}$ and $C_{0}$ is some sufficiently large constant that will be determined later.

Select $T_{0}$ such that for any $T \geq T_{0}$ we have $\left(T+t_{0}+\delta_{i n}+1\right)^{1-\varepsilon} \leq T \frac{\varepsilon}{12\left(1+2 \delta_{i n}+A_{i n}\right)}$ and $\left(T+t_{0}+\delta_{i n}+1\right)^{1-\varepsilon} \leq T \frac{\delta_{i n}}{12\left(1+2 \delta_{i n}+A_{i n}\right)}$ if $\delta_{i n}>0$. There are only a finite number of pairs $(T, N)$ with $T \leq T_{0}$, so we can select $C_{0}$ such that (3.5) holds for $T \leq T_{0}$.

Before proceeding with induction, we need to establish (3.5) for $d \geq T+t_{0}$ and any $T \geq T_{0}$. In this case, $T f_{d}(x)=O\left(T d^{-2+\varepsilon}\right)=O\left(d^{-1+\varepsilon}\right)$, so we need to prove that $E_{d}(T, N) \leq C\left(d, \frac{N}{T}\right)$ (and then increase $C_{0}$ to account for $\left.T f_{d}(x)\right)$. If $d>T+t_{0}$, it is trivial because $E_{d}(T, N)=0$. There can be only one vertex with in-degree $d=T+t_{0}$, and it exists only if $(\ddagger)$ has not been used after the first step and the same target vertex was selected at every step; the probability of that given $\# G=N+n_{0}$ is not greater than $\prod_{i=2}^{N} a \frac{T+t_{0}+\delta_{i n}}{T+t_{0}+\delta_{i n} i}=a^{N-1} \exp \sum_{i=2}^{N} \ln \left(1-\frac{\delta_{i n}(i-1)}{T+t_{0}+\delta_{i n} i}\right) \leq a^{N-1}\left(\exp \frac{\delta_{i n} N(N-1)}{2\left(T\left(1+\delta_{i n}\right)+t_{0}\right)}\right)^{-1} \leq$ $\frac{a^{N-1}}{1+\frac{\delta_{i n}^{N(N-1)}}{2\left(T\left(1+\delta_{i n}\right)+t_{0}\right)}}$. Because $\gamma+\delta_{i n}>0$, we have either $a<1$ or $\delta_{i n}>0$; in both cases $E_{d}(T, N)=O\left(\frac{1}{1+N^{2} / T}\right)$, so (3.5) holds for $T \geq T_{0}$.

Now assume that (3.5) is proved for some value of $T \geq T_{0}$ and consider the value $T+1$. Assume also that $d<T+1+t_{0}$.

Let $0 \leq N \leq T+1$ and $x=\frac{N}{T+1}$. If $N \neq T+1$, the inductive hypothesis and the Taylor formula imply that

$$
\begin{gathered}
E_{d}(T, N)=T f_{d}\left(x+\frac{x}{T}\right)+\theta_{1}=T f_{d}(x)+x f_{d}^{\prime}(x)+\frac{x^{2}}{2 T} f_{d}^{\prime \prime}(\xi)+\theta_{1} \\
=T f_{d}(x)+x f_{d}^{\prime}(x)+O\left(\frac{(d+1)^{-2+\varepsilon}}{T}\right)+\theta_{1}
\end{gathered}
$$

where $\left|\theta_{1}\right| \leq C\left(d, x+\frac{x}{T}\right)$. Similarly,

$$
E_{d-1}(T, N)=T f_{d-1}(x)+O\left((d+1)^{-2+\varepsilon}\right)+\theta_{2}
$$

for $d \geq 1$ with $\left|\theta_{2}\right| \leq C\left(d-1, x+\frac{x}{T}\right)$. The same holds for $d=0$ with $\theta_{2}=0$. 
If $N \neq 0$, we have for the same reasons

$$
\begin{gathered}
E_{d}(T, N-1)=T f_{d}\left(x-\frac{1-x}{T}\right)+\theta_{3}=T f_{d}(x)-(1-x) f_{d}^{\prime}(x)+\frac{(1-x)^{2}}{2 T} f_{d}^{\prime \prime}(\xi)+\theta_{3} \\
=T f_{d}(x)-(1-x) f_{d}^{\prime}(x)+O\left(\frac{(1-x)(d+1)^{-2+\varepsilon}}{T}\right)+\theta_{3}
\end{gathered}
$$

and $E_{d-1}(T, N-1)=T f_{d-1}(x)+O\left((1-x)(d+1)^{-2+\varepsilon}\right)+\theta_{4},\left|\theta_{3}\right| \leq C\left(d, x-\frac{1-x}{T}\right)$, $\theta_{4}=0$ for $d=0,\left|\theta_{4}\right| \leq C\left(d-1, x-\frac{1-x}{T}\right)$ for $d \geq 1$.

Now (3.1) becomes

$$
\begin{gathered}
E_{d}(T+1, N)=[d=0] a x+[d=1](1-a) x+x\left(T f_{d}(x)-(1-x) f_{d}^{\prime}(x)\right. \\
\left.+O\left(\frac{(1-x)(d+1)^{-2+\varepsilon}}{T}\right)\right)\left(1-\frac{a\left(d+\delta_{i n}\right)}{T\left(1+\delta_{i n} x\right)}+O\left(\frac{a}{T}\right)\right) \\
+a x\left(T f_{d-1}(x)+O\left((1-x)(d+1)^{-2+\varepsilon}\right)\right) \frac{d-1+\delta_{i n}}{T\left(1+\delta_{i n} x\right)}\left(1+O\left(\frac{1}{T}\right)\right) \\
+(1-x)\left(T f_{d}(x)+x f_{d}^{\prime}(x)+O\left(\frac{(d+1)^{-2+\varepsilon}}{T}\right)\right)\left(1-\frac{d+\delta_{i n}}{T\left(1+\delta_{i n} x\right)}+O\left(\frac{1}{T}\right)\right) \\
+(1-x)\left(T f_{d-1}(x)+O\left((d+1)^{-2+\varepsilon}\right)\right) \frac{d-1+\delta_{i n}}{T\left(1+\delta_{i n} x\right)}\left(1+O\left(\frac{1}{T}\right)\right) \\
+x \theta_{3}\left(1-\frac{a\left(d+\delta_{i n}\right)}{T\left(1+\delta_{i n} x\right)+\delta_{i n} x+A_{i n}-\delta_{i n}}\right)+a x \theta_{4} \frac{d-1+\delta_{i n}}{T\left(1+\delta_{i n} x\right)+\delta_{i n} x+A_{i n}-\delta_{i n}} \\
+(1-x) \theta_{1}\left(1-\frac{d-1+\delta_{i n}}{T\left(1+\delta_{i n} x\right)+\delta_{i n} x+A_{i n}}\right)+(1-x) \theta_{2} \frac{d\left(1+\delta_{i n} x\right)+\delta_{i n} x+A_{i n}}{T(n)}
\end{gathered}
$$

we have dropped indicators $[N>0]$ and $[N<T+1]$ because everything on the right-hand side is defined as $x=0$ and $x=1$, too, and corresponding terms are zero, anyway, because of factors $x$ and $1-x$.

Expand (3.6):

$$
\begin{aligned}
& E_{d}(T+1, N)=T f_{d}(x)+[d=0] a x+[d=1](1-a) x \\
& \quad-x f_{d}(x) \frac{a\left(d+\delta_{i n}\right)}{1+\delta_{i n} x}+a x f_{d-1}(x) \frac{d-1+\delta_{i n}}{1+\delta_{i n} x}-(1-x) f_{d}(x) \frac{d+\delta_{i n}}{1+\delta_{i n} x} \\
& +(1-x) f_{d-1}(x) \frac{d-1+\delta_{i n}}{1+\delta_{i n} x}+O\left(\frac{(1-x)(d+1)^{-1+\varepsilon}}{T}\right)+O\left(\frac{a x(d+1)^{-1+\varepsilon}}{T}\right) \\
& +x \theta_{3}\left(1-\frac{a\left(d+\delta_{i n}\right)}{T\left(1+\delta_{i n} x\right)+\delta_{i n} x+A_{i n}-\delta_{i n}}\right)+a x \theta_{4} \frac{d-1+\delta_{i n}}{T\left(1+\delta_{i n} x\right)+\delta_{i n} x+A_{i n}-\delta_{i n}} \\
& +(1-x) \theta_{1}\left(1-\frac{d+\delta_{i n}}{T\left(1+\delta_{i n} x\right)+\delta_{i n} x+A_{i n}}\right)+(1-x) \theta_{2} \frac{d-1+\delta_{i n}}{T\left(1+\delta_{i n} x\right)+\delta_{i n} x+A_{i n}} .
\end{aligned}
$$


The sum of terms without $\theta_{i}$ and $O(\cdot)$ equals $(T+1) f_{d}(x)$ from (3.2). Denote the sum of other terms as $\theta$. Then,

$$
E_{d}(T+1, N)=(T+1) f_{d}(x)+\theta,
$$

so we need to prove that $|\theta| \leq C(d, x)$.

The expression $x\left(1-\frac{a\left(d+\delta_{i n}\right)}{T\left(1+\delta_{i n} x\right)+\delta_{i n} x+A_{\text {in }}-\delta_{\text {in }}}\right)=\frac{N}{T+1}\left(1-\frac{a\left(d+\delta_{i n}\right)}{T+\delta_{\text {in }} N+t_{0}+\delta_{i n} n_{0}-\delta_{i n}}\right)$ is always nonnegative (provided that $d \leq T+t_{0}$ ). The expression $1-\frac{d+\delta_{i n}}{T\left(1+\delta_{i n} x\right)+\delta_{i n} x+A_{i n}}=1-$ $\frac{d+\delta_{i n}}{T+\delta_{i n} N+t_{0}+\delta_{i n} n_{0}}$ is also always nonnegative. Therefore,

$$
\begin{aligned}
|\theta| & \leq x C\left(d, x-\frac{1-x}{T}\right)+(1-x) C\left(d, x+\frac{x}{T}\right) \\
& -\frac{a x}{T\left(1+\delta_{i n} x\right)+\delta_{i n} x+A_{i n}-\delta_{i n}}\left(\left(d+\delta_{i n}\right) C\left(d, x-\frac{1-x}{T}\right)\right. \\
& \left.-[d \geq 1]\left(d-1+\delta_{i n}\right) C\left(d-1, x-\frac{1-x}{T}\right)\right)-\frac{1-x}{T\left(1+\delta_{i n} x\right)+\delta_{i n} x+A_{i n}} \\
& \times\left(\left(d+\delta_{i n}\right) C\left(d, x+\frac{x}{T}\right)-[d \geq 1]\left(d-1+\delta_{i n}\right) C\left(d-1, x+\frac{x}{T}\right)\right) \\
& +O\left(\frac{1-x+a x}{(d+1)^{1-\varepsilon} T}\right) .
\end{aligned}
$$

We have $\left|C^{\prime \prime}(d, x)\right|=C_{0}\left|-\frac{2\left(d+\delta_{i n}+1\right)^{1-\varepsilon}}{\left(1+\left(d+\delta_{i n}+1\right)^{1-\varepsilon} x^{2}\right)^{2}}+\frac{8\left(d+\delta_{i n}+1\right)^{2-2 \varepsilon} x^{2}}{\left(1+\left(d+\delta_{i n}+1\right)^{1-\varepsilon} x^{2}\right)^{3}}\right| \leq$ $C_{0} \frac{6\left(d+\delta_{i n}+1\right)^{1-\varepsilon}}{\left(1+\left(d+\delta_{i n}+1\right)^{1-\varepsilon} x^{2}\right)^{2}} \leq 6\left(d+\delta_{i n}+1\right)^{1-\varepsilon} C(d, x)$. Using the Taylor formula, we obtain

$$
\begin{gathered}
\left|x C\left(d, x-\frac{1-x}{T}\right)+(1-x) C\left(d, x+\frac{x}{T}\right)-C(d, x)\right| \\
=\left|\frac{x(1-x)^{2}}{2 T^{2}} C^{\prime \prime}\left(d, \xi_{1}\right)+\frac{(1-x) x^{2}}{2 T^{2}} C^{\prime \prime}\left(d, \xi_{2}\right)\right| \\
\leq \frac{3 x(1-x)\left(d+\delta_{i n}+1\right)^{1-\varepsilon} C\left(d, x-\frac{1-x}{T}\right)}{T^{2}} .
\end{gathered}
$$

If $d \geq 1$, then

$$
\begin{aligned}
& \left(d+\delta_{i n}\right) C(d, x)-\left(d-1+\delta_{i n}\right) C(d-1, x) \\
& =C_{0} \int_{d-1}^{d}\left(\frac{z+\delta_{i n}}{1+\left(z+\delta_{i n}+1\right)^{1-\varepsilon} x^{2}}\right)_{z}^{\prime} d z \\
& =C_{0} \int_{d-1}^{d} \frac{1+\varepsilon\left(z+\delta_{i n}+1\right)^{1-\varepsilon} x^{2}+(1-\varepsilon)\left(z+\delta_{i n}+1\right)^{-\varepsilon} x^{2}}{\left(1+\left(z+\delta_{i n}+1\right)^{1-\varepsilon} x^{2}\right)^{2}} d z \\
& \geq C_{0} \frac{\varepsilon}{1+\left(d+\delta_{i n}+1\right)^{1-\varepsilon} x^{2}}=\varepsilon C(d, x) .
\end{aligned}
$$


Thus, for $d \geq 1$

$$
\begin{aligned}
& |\theta| \leq C(d, x)+\frac{3 x(1-x)\left(d+\delta_{i n}+1\right)^{1-\varepsilon} C\left(d, x-\frac{1-x}{T}\right)}{T^{2}} \\
& -\frac{a x \varepsilon C\left(d, x-\frac{1-x}{T}\right)}{T\left(1+\delta_{i n} x\right)+\delta_{i n} x+A_{i n}-\delta_{i n}}-\frac{(1-x) \varepsilon C\left(d, x+\frac{x}{T}\right)}{T\left(1+\delta_{i n} x\right)+\delta_{i n} x+A_{i n}}+O\left(\frac{1-x+a x}{(d+1)^{1-\varepsilon} T}\right) .
\end{aligned}
$$

Because $T \geq T_{0}$ and $d \leq T+t_{0}$, we have $\left(d+\delta_{i n}+1\right)^{1-\varepsilon} \leq T \frac{\varepsilon}{12}$,

$$
\frac{C\left(d, x-\frac{1-x}{T}\right)}{C\left(d, x+\frac{x}{T}\right)}=\frac{1+\left(d+\delta_{i n}+1\right)^{1-\varepsilon}\left(x+\frac{x}{T}\right)^{2}}{1+\left(d+\delta_{i n}+1\right)^{1-\varepsilon}\left(x-\frac{1-x}{T}\right)^{2}} \leq 1+\left(d+\delta_{i n}+1\right)^{1-\varepsilon} \frac{2\left(x+\frac{x}{T}\right)}{T} \leq 2,
$$

so, $\frac{3 x(1-x)\left(d+\delta_{i n}+1\right)^{1-\varepsilon} C\left(d, x-\frac{1-x}{T}\right)}{T^{2}} \leq \frac{\varepsilon(1-x) C\left(d, x+\frac{x}{T}\right)}{2 T\left(1+2 \delta_{i n}+A_{\text {in }}\right)} \leq \frac{\varepsilon(1-x) C\left(d, x+\frac{x}{T}\right)}{2\left(T\left(1+\delta_{\text {in }} x\right)+\delta_{\text {in }} x+A_{\text {in }}\right)}$. Because $C(d, x) \geq$ $\frac{C_{0}}{2\left(d+\delta_{i n}+1\right)^{1-\varepsilon}}$,

$$
|\theta| \leq C(d, x)-\frac{(1-x+a x) \varepsilon C_{0}}{4\left(d+\delta_{i n}+1\right)^{1-\varepsilon}\left(T\left(1+\delta_{i n} x\right)+\delta_{i n} x+A_{i n}\right)}+O\left(\frac{1-x+a x}{(d+1)^{1-\varepsilon} T}\right) .
$$

Take $C_{0}$ such that $\frac{(1-x+a x) \varepsilon C_{0}}{4\left(d+\delta_{i n}+1\right)^{1-\varepsilon}\left(T\left(1+\delta_{i n} x\right)+\delta_{i n} x+A_{i n}\right)} \geq O\left(\frac{1-x+a x}{(d+1)^{1-\varepsilon} T}\right)$, then (3.5) follows.

If $d=0$ and $\delta_{i n}=0,(3.5)$ was already proved. The case $d=0$ and $\delta_{i n}>0$ is similar to the case $d \geq 1$ with the factor $\varepsilon$ from (3.7) replaced with $\delta_{i n}$.

Lemma 3.2. Let $n$ be a random variable that takes the value $N$ with probability $\operatorname{Pr}(n=$ $N)=\operatorname{Pr}\left(\# G_{T}=N+n_{0}\right)$. Let $f(x) \in C^{2}[0,1]$. Then

$$
\mathbf{E} f\left(\frac{n}{T}\right)=f(\alpha+\gamma)+O\left(\frac{\max _{0 \leq x \leq 1}\left|f^{\prime \prime}(x)\right|}{T}\right) \text {. }
$$

Proof. Obviously, E1 $=1$ and $\mathbf{E} n=(\alpha+\gamma) T$. Furthermore,

$$
\begin{aligned}
\mathbf{E}(n(n-1)) & =\sum_{n=0}^{T} n(n-1)\left(\begin{array}{l}
T \\
n
\end{array}\right)(\alpha+\gamma)^{n} \beta^{T-n}=\sum_{n=2}^{T} T(T-1)\left(\begin{array}{c}
T \\
n-2
\end{array}\right)(\alpha+\gamma)^{n} \beta^{T-n} \\
& =T(T-1)(\alpha+\gamma)^{2} .
\end{aligned}
$$

Hence,

$$
\mathbf{E}\left(\frac{n}{T}\right)^{2}=(\alpha+\gamma)^{2}\left(1-\frac{1}{T}\right)+\frac{\alpha+\gamma}{T}
$$


For any $x \in[0,1]$, we have $f(x)=f(\alpha+\gamma)+(x-\alpha-\gamma) f^{\prime}(\alpha+\gamma)+\frac{(x-\alpha-\gamma)^{2}}{2} f^{\prime \prime}(\xi)$, where $\xi$ is some point between $x$ and $\alpha+\gamma$. Therefore,

$$
\begin{aligned}
\left|\mathbf{E} f\left(\frac{n}{T}\right)-f(\alpha+\gamma)\right| & =\left|\mathbf{E}\left(\frac{1}{2}\left(\frac{n}{T}-\alpha-\gamma\right)^{2} f^{\prime \prime}(\xi)\right)\right| \\
& \leq \frac{(\alpha+\gamma)-(\alpha+\gamma)^{2}}{2 T} \max _{0 \leq x \leq 1}\left|f^{\prime \prime}(x)\right| .
\end{aligned}
$$

Now, the theorem in the case $\gamma=0$ follows from Lemma 3.1 and Lemma 3.2. However, in the case $\gamma>0$ we need a slightly more subtle approach to estimate the remainder term. The Azuma-Hoeffding inequality implies that (in terms of Lemma 3.2) $\operatorname{Pr}(n<$ $(\alpha+\gamma-\lambda) T) \leq \exp \left(-T \lambda^{2} / 2\right)$ for $\lambda>0$, so $\mathbf{E}_{\frac{1}{1+\left(d+\delta_{i n}+1\right)^{1-\varepsilon} n^{2} / T^{2}}}=\mathbf{E} \frac{\left[\frac{n}{\frac{n}{L}} \leq \frac{\alpha+\gamma}{2}\right]}{1+\left(d+\delta_{i n}+1\right)^{1-\varepsilon} n^{2} / T^{2}}+$ $\mathbf{E}_{\frac{\left[\frac{n}{T} \geq \frac{\alpha+\gamma}{2}\right]}{1+\left(d+\delta_{i n}+1\right)^{1-\varepsilon} n^{2} / T^{2}}} \leq \exp \left(-T \frac{(\alpha+\gamma)^{2}}{8}\right)+\frac{1}{1+\left(d+\delta_{i n}+1\right)^{1-\varepsilon} \frac{(\alpha+\gamma)^{2}}{4}}=O\left(d^{-1+\varepsilon}\right)$ if $d \leq T+t_{0}$. For $d>T+t_{0}$, the theorem holds because $n_{\text {in }}=0$.

\section{CONCENTRATION FOR NUMBER OF VERTICES}

Let $D_{d_{1}, d_{2}}(T, N)=\mathbf{E}\left(n_{i n}\left(G, d_{1}\right) n_{i n}\left(G, d_{2}\right) \mid G \in \mathcal{G}\left(T+t_{0}\right), \# G=N+n_{0}\right)-\left[d_{1}=\right.$ $\left.d_{2}\right] E_{d_{1}}(T, N)$.

$$
\begin{aligned}
D_{d_{1}, d_{2}}(T, N)= & \sum_{i, j=1}^{N+n_{0}} \operatorname{Pr}\left(d_{i n}(G, i)=d_{1}, d_{i n}(G, j)=d_{2} \mid G \in \mathcal{G}\left(T+t_{0}\right), \# G=N+n_{0}\right) \\
& -\left[d_{1}=d_{2}\right] E_{d_{1}}(T, N) \sum_{\substack{i, j=1 \\
i \neq j}}^{N+n_{0}} \operatorname{Pr}\left(d_{i n}(G, i)\right. \\
= & \left.d_{1}, d_{i n}(G, j)=d_{2} \mid G \in \mathcal{G}\left(T+t_{0}\right), \# G=N+n_{0}\right) .
\end{aligned}
$$

Lemma 4.1. If $\gamma+\delta_{\text {in }}>0$, then

$$
\begin{aligned}
D_{d_{1}, d_{2}}(T, N)= & T^{2} f_{d_{1}}\left(\frac{N}{T}\right) f_{d_{2}}\left(\frac{N}{T}\right) \\
& +O\left(\frac{T\left(d_{1}+\delta_{i n}+1\right)^{-1-\frac{1}{c_{i n}(N / T)+\varepsilon}}}{1+\left(d_{2}+\delta_{i n}+1\right)^{1-\varepsilon}(N / T)^{2}}+\frac{T\left(d_{2}+\delta_{i n}+1\right)^{-1-\frac{1}{c_{i n}(N / T)+\varepsilon}}}{1+\left(d_{1}+\delta_{i n}+1\right)^{1-\varepsilon}(N / T)^{2}}\right. \\
& \left.+\frac{1}{\left(1+\left(d_{1}+\delta_{i n}+1\right)^{1-\varepsilon}(N / T)^{2}\right)\left(1+\left(d_{2}+\delta_{i n}+1\right)^{1-\varepsilon}(N / T)^{2}\right)}\right) .
\end{aligned}
$$


Proof. The recurrent equation for $D_{d_{1}, d_{2}}(T, N)$ is obtained similarly to (3.1): for $i, j \in G_{T}$, $i \neq j$,

$$
\begin{aligned}
\operatorname{Pr}\left(d_{i n}\left(G_{T+1}, i\right)=\right. & \left.d_{1}, d_{i n}\left(G_{T+1}, j\right)=d_{2}, \# G_{T+1}=N+n_{0}, \dagger\right) \\
= & \alpha \operatorname{Pr}\left(d_{i n}\left(G_{T}, i\right)=d_{1}, d_{i n}\left(G_{T}, j\right)=d_{2}, \# G_{T}=N+n_{0}-1\right) \\
& \times\left(1-\frac{d_{1}+d_{2}+2 \delta_{i n}}{T+\delta_{i n} N+A_{i n}-\delta_{i n}}\right) \\
& +\alpha \operatorname{Pr}\left(d_{i n}\left(G_{T}, i\right)=d_{1}-1, d_{i n}\left(G_{T}, j\right)=d_{2}, \# G_{T}=N+n_{0}-1\right) \\
& \times \frac{d_{1}-1+\delta_{i n}}{T+\delta_{i n} N+A_{i n}-\delta_{i n}}+\alpha \operatorname{Pr}\left(d_{i n}\left(G_{T}, i\right)=d_{1}, d_{i n}\left(G_{T}, j\right)\right. \\
= & \left.d_{2}-1, \# G_{T}=N+n_{0}-1\right) \frac{d_{2}-1+\delta_{i n}}{T+\delta_{i n} N+A_{i n}-\delta_{i n}},
\end{aligned}
$$

$\operatorname{Pr}\left(d_{i n}\left(G_{T+1}, i\right)=d_{1}, d_{i n}\left(G_{T+1}, j\right)=d_{2}, \# G_{T+1}=N+n_{0}, \dagger \dagger\right)$

$$
\begin{aligned}
= & \beta \operatorname{Pr}\left(d_{i n}\left(G_{T}, i\right)=d_{1}, d_{i n}\left(G_{T}, j\right)=d_{2}, \# G_{T}=N+n_{0}\right) \\
& \times\left(1-\frac{d_{1}+d_{2}+2 \delta_{i n}}{T+\delta_{i n} N+A_{\text {in }}}\right)+\beta \operatorname{Pr}\left(d_{i n}\left(G_{T}, i\right)=d_{1}-1,\right.
\end{aligned}
$$$$
\left.d_{i n}\left(G_{T}, j\right)=d_{2}, \# G_{T}=N+n_{0}\right) \frac{d_{1}-1+\delta_{i n}}{T+\delta_{i n} N+A_{i n}}+\beta \operatorname{Pr}\left(d_{i n}\left(G_{T}, i\right)\right.
$$$$
\left.=d_{1}, d_{i n}\left(G_{T}, j\right)=d_{2}-1, \# G_{T}=N+n_{0}\right) \frac{d_{2}-1+\delta_{i n}}{T+\delta_{i n} N+A_{i n}},
$$

$$
\begin{aligned}
\operatorname{Pr}\left(d_{i n}\left(G_{T+1}, i\right)=\right. & \left.d_{1}, d_{i n}\left(G_{T+1}, j\right)=d_{2}, \# G_{T+1}=N+n_{0}, \ddagger\right) \\
= & \gamma \operatorname{Pr}\left(d_{i n}\left(G_{T}, i\right)=d_{1}, d_{i n}\left(G_{T}, j\right)=d_{2}, \# G_{T}=N+n_{0}-1\right) ; \\
D_{d_{1}, d_{2}}(T+1, N)= & {\left[N>0, d_{1}=0\right] \alpha E_{d_{2}}(T, N-1) \frac{N}{(\alpha+\gamma)(T+1)} } \\
& +\left[N>0, d_{2}=0\right] \alpha E_{d_{1}}(T, N-1) \frac{N}{(\alpha+\gamma)(T+1)} \\
& +[N>0] \alpha D_{d_{1}, d_{2}}(T, N-1)\left(1-\frac{d_{1}+d_{2}+2 \delta_{i n}}{T+\delta_{i n} N+A_{i n}-\delta_{i n}}\right) \\
& \times \frac{N}{(\alpha+\gamma)(T+1)}+[N>0] \alpha D_{d_{1}-1, d_{2}}(T, N-1) \\
& \times \frac{d_{1}-1+\delta_{i n}}{T+\delta_{i n} N+A_{i n}-\delta_{i n}} \frac{N+\gamma)(T+1)}{\left(\alpha+d_{i n}\right.} \\
& \left.+[N>0] \alpha D_{d_{1}, d_{2}-1}(T, N-1) \frac{d_{2}-1+\delta_{i n}}{T+\delta_{i n} N+A_{i n}-\delta_{i n}} \frac{N+\gamma)(T+1)}{\left(\alpha+d_{i n}\right.}\right) \frac{T+1-N}{\beta(T+1)} \\
& +[N<T+1] \beta D_{d_{1}, d_{2}}(T, N)\left(1-\frac{d_{1}+d_{2}+2 \delta_{i n}}{T+\delta_{i n} N+A_{i n}}\right)
\end{aligned}
$$




$$
\begin{aligned}
& +[N<T+1] \beta D_{d_{1}-1, d_{2}}(T, N) \frac{d_{1}-1+\delta_{i n}}{T+\delta_{i n} N+A_{i n}} \frac{T+1-N}{\beta(T+1)} \\
& +[N<T+1] \beta D_{d_{1}, d_{2}-1}(T, N) \frac{d_{2}-1+\delta_{i n}}{T+\delta_{i n} N+A_{i n}} \frac{T+1-N}{\beta(T+1)} \\
& +\left[N>0, d_{1}=1\right] \gamma E_{d_{2}}(T, N-1) \frac{N}{(\alpha+\gamma)(T+1)} \\
& +\left[N>0, d_{2}=1\right] \gamma E_{d_{1}}(T, N-1) \frac{N}{(\alpha+\gamma)(T+1)} \\
& +[N>0] \gamma D_{d_{1}, d_{2}}(T, N-1) \frac{N}{(\alpha+\gamma)(T+1)} .
\end{aligned}
$$

We use induction by $T$ and, for fixed $T$, by $d_{1}+d_{2}$ to prove the formula

$$
\left|D_{d_{1}, d_{2}}(T, N)-T^{2} f_{d_{1}}\left(\frac{N}{T}\right) f_{d_{2}}\left(\frac{N}{T}\right)\right| \leq C\left(T, d_{1}, d_{2}, \frac{N}{T}\right),
$$

$C\left(T, d_{1}, d_{2}, x\right)=T C_{1}\left(d_{1}, d_{2}, x\right)+T C_{2}\left(d_{1}, d_{2}, x\right)+C_{3}\left(d_{1}, d_{2}, x\right)$,

$$
\begin{aligned}
C_{1}\left(d_{1}, d_{2}, x\right) & =C_{0} \frac{\hat{f}_{d_{1}}(x)}{1+\left(d_{2}+\delta_{i n}+1\right)^{1-\varepsilon} x^{2}}, \\
C_{2}\left(d_{1}, d_{2}, x\right) & =C_{0} \frac{\hat{f}_{d_{2}}(x)}{1+\left(d_{1}+\delta_{i n}+1\right)^{1-\varepsilon} x^{2}}, \\
C_{3}\left(d_{1}, d_{2}, x\right) & =\frac{C_{0}\left[d_{1} \geq 1, d_{2} \geq 1\right]}{\left(1+\left(d_{1}+\delta_{i n}+1\right)^{1-\varepsilon} x^{2}\right)\left(1+\left(d_{2}+\delta_{i n}+1\right)^{1-\varepsilon} x^{2}\right)}, \\
\hat{f}_{0}(x) & =1, \\
\hat{f}_{d}(x) & =\prod_{i=2}^{d} \frac{i+\delta_{i n}-1}{i+\delta_{i n}+\frac{1}{c_{i n}(x)+\varepsilon}} \text { for } d \geq 1 .
\end{aligned}
$$

Because $\frac{i+\delta_{i n}-1}{i+\delta_{i n}+\frac{1}{c_{i n}(x)+\varepsilon}}=1-\left(1+\frac{1}{c_{i n}(x)+\varepsilon}\right) \frac{1}{i+\delta_{i n}+\frac{1}{c_{i n}(x)+\varepsilon}} \leq\left(1+\frac{1}{i+\delta_{i n}+\frac{1}{c_{i n}(x)+\varepsilon}}\right)^{-1-\frac{1}{c_{i n}(x)+\varepsilon}}$ and $\frac{i+\delta_{i n}-1}{i+\delta_{i n}+\frac{1}{c_{i n}(x)+\varepsilon}}=\left(1+\left(1+\frac{1}{c_{i n}(x)+\varepsilon}\right) \frac{1}{i+\delta_{i n}-1}\right)^{-1} \geq\left(1+\frac{1}{i+\delta_{i n}-1}\right)^{-1-\frac{1}{c_{i n}(x)+\varepsilon}}$, we have $(1+$ $\left.\delta_{i n}\right)^{1+\frac{1}{1+\varepsilon}}\left(d+\delta_{i n}\right)^{-1-\frac{1}{c_{i n}(x)+\varepsilon}} \leq \hat{f}_{d}(x) \leq\left(2+\delta_{i n}+\frac{1}{\varepsilon}\right)^{1+\frac{1}{\varepsilon}}\left(d+\delta_{i n}+1+\frac{1}{1+\varepsilon}\right)^{-1-\frac{1}{c_{i n}(x)+\varepsilon}}$, so $\hat{f}_{d}(x)=\Theta\left(\left(d+\delta_{i n}+1\right)^{-1-\frac{1}{c_{i n}(x)+\varepsilon}}\right)$, where both implied constants depend only on $\varepsilon$ and parameters of the model. For the same reasons as for $f_{d}(x)$, we have $\frac{\hat{f}_{d}^{\prime}(x)}{\hat{f}_{d}(x)}=O\left(\ln \left(d+\delta_{i n}+1\right)\right)$ and $\frac{\hat{f}_{d}^{\prime \prime}(x)}{\hat{f}_{d}(x)}=O\left(\ln ^{2}\left(d+\delta_{i n}+1\right)\right)$.

Since $\left|\frac{d}{d x}\left(\frac{1}{1+\lambda x^{2}}\right)\right|=\frac{2 \lambda x}{\left(1+\lambda x^{2}\right)^{2}} \leq \frac{\lambda x}{\sqrt{\lambda x^{2}}\left(1+\lambda x^{2}\right)}=\sqrt{\lambda} \frac{1}{1+\lambda x^{2}}$ and $\left|\frac{d^{2}}{d x^{2}}\left(\frac{1}{1+\lambda x^{2}}\right)\right| \leq 6 \lambda \frac{1}{1+\lambda x^{2}}$, we have $\frac{C_{i}^{\prime}\left(d_{1}, d_{2}, x\right)}{C_{i}\left(d_{1}, d_{2}, x\right)}=O\left(\left(d_{1}+\delta_{i n}+1\right)^{\frac{1-\varepsilon}{2}}+\left(d_{2}+\delta_{i n}+1\right)^{\frac{1-\varepsilon}{2}}\right)$ and $\frac{C_{i}^{\prime \prime}\left(d_{1}, d_{2}, x\right)}{C_{i}\left(d_{1}, d_{2}, x\right)}=O\left(\left(d_{1}+\right.\right.$ $\left.\left.\delta_{i n}+1\right)^{1-\varepsilon}+\left(d_{2}+\delta_{i n}+1\right)^{1-\varepsilon}\right)$.

If $\xi \in\left[x-\frac{1-x}{T}, x+\frac{x}{T}\right]$ and $d \leq T+t_{0}$, we have $\hat{f}_{d}^{\prime}(\xi)=O\left(\left(d+1+\delta_{i n}\right)^{-1-\frac{1}{c_{i n}(\xi)+\varepsilon}} \ln (d+\right.$ $\left.\left.1+\delta_{i n}\right)\right)=O\left(\left(d+1+\delta_{i n}\right)^{-1-\frac{1}{c_{i n}(x)+\varepsilon}}+O\left(\frac{1}{T}\right) \ln \left(d+1+\delta_{i n}\right)\right)=O\left(\hat{f}_{d}(x) \ln (d+1+\right.$ 
$\left.\left.\delta_{i n}\right) e^{O\left(\frac{\ln \left(d+1+\delta_{i n}\right)}{T}\right)}\right)=O\left(\hat{f}_{d}(x) \ln \left(d+1+\delta_{i n}\right)\right), \max \left\{\left|\hat{f}_{d}\left(x-\frac{1-x}{T}\right)-\hat{f}_{d}(x)\right|, \mid \hat{f}_{d}\left(x+\frac{x}{T}\right)-\right.$ $\left.\hat{f}_{d}(x) \mid\right\} \quad \leq \frac{1}{T} \max _{\xi \in\left[x-\frac{1-x}{T}, x+\frac{x}{T}\right]}\left|\hat{f}_{d}^{\prime}(\xi)\right|=\hat{f}_{d}(x) O\left(\frac{\ln \left(d+1+\delta_{i n}\right)}{T}\right), \hat{f}_{d}\left(x-\frac{1-x}{T}\right)=\hat{f}_{d}(x)(1+$ $\left.O\left(\frac{\ln \left(d+1+\delta_{i n}\right)}{T}\right)\right)$ and $\hat{f}_{d}\left(x+\frac{x}{T}\right)=\hat{f}_{d}(x)\left(1+O\left(\frac{\ln \left(d+1+\delta_{i n}\right)}{T}\right)\right)$. Moreover, $\left|\frac{1+\lambda x^{2}}{1+\lambda \xi^{2}}-1\right| \leq \frac{2 \lambda|\xi-x|}{1+\lambda \xi^{2}} \leq$ $\sqrt{\lambda}|\xi-x|$, so $C_{i}\left(d_{1}, d_{2}, x+\frac{x}{T}\right)=C_{i}\left(d_{1}, d_{2}, x\right)\left(1+O\left(\frac{\left(d_{1}+1+\delta_{i n}\right)^{\frac{1-\varepsilon}{2}}+\left(d_{2}+1+\delta_{i n}\right)^{\frac{1-\varepsilon}{2}}}{T}\right)\right)$ and $C_{i}\left(d_{1}, d_{2}, x-\frac{1-x}{T}\right)=C_{i}\left(d_{1}, d_{2}, x\right)\left(1+O\left(\frac{\left(d_{1}+1+\delta_{i n}\right)^{\frac{1-\varepsilon}{2}}+\left(d_{2}+1+\delta_{i n}\right)^{\frac{1-\varepsilon}{2}}}{T}\right)\right)$.

Select $T_{0}$ such that, for $T \geq T_{0}$ and $d_{1}+d_{2} \leq T+t_{0}$, the following inequalities hold:

$$
\begin{aligned}
\left|C_{i}\left(d_{1}, d_{2}, x-\frac{1-x}{T}\right)-C_{i}\left(d_{1}, d_{2}, x\right)\right| & \leq \frac{\varepsilon}{3} C_{i}\left(d_{1}, d_{2}, x\right), \\
\left|C_{i}\left(d_{1}, d_{2}, x+\frac{x}{T}\right)-C_{i}\left(d_{1}, d_{2}, x\right)\right| & \leq \frac{\varepsilon}{3} C_{i}\left(d_{1}, d_{2}, x\right), \\
\left|C_{i}^{\prime \prime}\left(d_{1}, d_{2}, x\right)\right| & \leq \frac{\varepsilon}{3\left(1+2 \delta_{i n}+A_{i n}\right)} T C_{i}\left(d_{1}, d_{2}, x\right) .
\end{aligned}
$$

(Note that validity of these inequalities does not depend on $C_{0}$, so $T_{0}$ does not depend on $C_{0}$, which has not been selected yet.)

There are only a finite number of pairs $(T, N)$ with $T \leq T_{0}$, so it is possible to select $C_{0}$ such that (4.2) holds for $T \leq T_{0}$. This is the base of induction.

Before proceeding with induction, we need to establish (4.2) for the cases when $1-\frac{d_{1}+d_{2}+2 \delta_{i n}}{T-1+\delta_{i n} N+t_{0}+\delta_{i n} n_{0}-\delta_{i n}}<0$. If $d_{1}+d_{2}>T+t_{0}$, then $D_{d_{1}, d_{2}}(T, N)=0$, so (4.2) holds. If $N=O(1)$, then the bound $D_{d_{1}, d_{2}} \leq\left(N+n_{0}\right)^{2}=O(1)$ is sufficient. The only other case when the expression above can be negative is $d_{1}+d_{2}=T+t_{0}$ and $\delta_{i n}=0$. Then, $\gamma>0$ and $a<1$; since $d_{1}+d_{2}=T+t_{0}$ means that ( $\ddagger$ ) has been used at most once, not counting the first step, the probability of that given $\# G_{T}=N+n_{0}$ is not greater than $N a^{N-2}$, so, $D_{d_{1}, d_{2}}(T, N) \leq\left(N+n_{0}\right) N a^{N-2}=O\left(\frac{1}{1+N}\right)$, which is less than $C\left(T, d_{1}, d_{2}, x\right)$ if $C_{0}$ is sufficiently large.

Now assume that (4.2) is proved for some value of $T \geq T_{0}$ and consider the value $T+1$. Assume also that $1-\frac{d_{1}+d_{2}+2 \delta_{i n}}{T+\delta_{i n} N+t_{0}+\delta_{i n} n_{0}-\delta_{i n}} \geq 0$. Let $0 \leq N \leq T+1$ and $x=\frac{N}{T+1}$.

If $\xi \in\left[x-\frac{1-x}{T}, x+\frac{x}{T}\right]$ and $d \leq T+t_{0}$, we have

$$
\begin{aligned}
& f_{d}^{\prime}(\xi)=O\left(\left(d+1+\delta_{i n}\right)^{-1-\frac{1}{c_{i n}(\xi)+\varepsilon}}\right)=O\left(\left(d+1+\delta_{i n}\right)^{-1-\frac{1}{c_{i n}(x)+\varepsilon}+O\left(\frac{1}{T}\right)}\right) \\
& =O\left(\left(d+1+\delta_{i n}\right)^{-1-\frac{1}{c_{i n}(x)+\varepsilon}}\right) e^{O\left(\frac{\ln \left(d+1+\delta_{i n}\right)}{T}\right)}=O\left(\left(d+1+\delta_{i n}\right)^{-1-\frac{1}{c_{i n}(x)+\varepsilon}}\right)
\end{aligned}
$$

and $f_{d}^{\prime \prime}(\xi)=O\left(\left(d+1+\delta_{i n}\right)^{-1-\frac{1}{c_{i n}(x)+\varepsilon}}\right)$ for the same reason.

If $N \neq T+1$, the inductive hypothesis and the Taylor formula imply that

$$
\begin{aligned}
D_{d_{1}, d_{2}}(T, N)= & T^{2} f_{d_{1}}\left(x+\frac{x}{T}\right) f_{d_{2}}\left(x+\frac{x}{T}\right)+\theta_{1} \\
= & T^{2} f_{d_{1}}(x) f_{d_{2}}(x)+x T\left(f_{d_{1}}(x) f_{d_{2}}(x)\right)^{\prime} \\
& \left.+O\left(\left(d_{1}+1+\delta_{i n}\right)^{-1-\frac{1}{c_{i n}(x)+\varepsilon}}\left(d_{2}+1+\delta_{i n}\right)^{-1-\frac{1}{c_{i n}(x)+\varepsilon}}\right)\right)+\theta_{1},
\end{aligned}
$$




$$
\begin{aligned}
D_{d_{1}-1, d_{2}}(T, N)= & T^{2} f_{d_{1}-1}(x) f_{d_{2}}(x) \\
& \left.+O\left(T\left(d_{1}+1+\delta_{i n}\right)^{-1-\frac{1}{c_{i n}(x)+\varepsilon}}\left(d_{2}+1+\delta_{i n}\right)^{-1-\frac{1}{c_{i n}(x)+\varepsilon}}\right)\right)+\theta_{2}, \\
D_{d_{1}, d_{2}-1}(T, N)= & T^{2} f_{d_{1}}(x) f_{d_{2}-1}(x) \\
& \left.+O\left(T\left(d_{1}+1+\delta_{i n}\right)^{-1-\frac{1}{c_{i n}(x)+\varepsilon}}\left(d_{2}+1+\delta_{i n}\right)^{-1-\frac{1}{c_{i n}(x)+\varepsilon}}\right)\right)+\theta_{3},
\end{aligned}
$$

where $\left|\theta_{1}\right| \leq C\left(T, d_{1}, d_{2}, x+\frac{x}{T}\right),\left|\theta_{2}\right| \leq C\left(T, d_{1}-1, d_{2}, x+\frac{x}{T}\right)$ if $d_{1} \geq 1$ and $\theta_{2}=0$ if $d_{1}=0,\left|\theta_{3}\right| \leq C\left(T, d_{1}, d_{2}-1, x+\frac{x}{T}\right)$ if $d_{2} \geq 1$ and $\theta_{3}=0$ if $d_{2}=0$. If $N \neq 0$, similarly,

$$
\begin{aligned}
D_{d_{1}, d_{2}}(T, N-1)= & T^{2} f_{d_{1}}\left(x-\frac{1-x}{T}\right) f_{d_{2}}\left(x-\frac{1-x}{T}\right)+\theta_{4} \\
= & T^{2} f_{d_{1}}(x) f_{d_{2}}(x)-(1-x) T\left(f_{d_{1}}(x) f_{d_{2}}(x)\right)^{\prime} \\
& \left.+O\left(\left(d_{1}+1+\delta_{i n}\right)^{-1-\frac{1}{c_{i n}(x)+\varepsilon}}\left(d_{2}+1+\delta_{i n}\right)^{-1-\frac{1}{c_{i n}(x)+\varepsilon}}\right)\right)+\theta_{4}, \\
D_{d_{1}-1, d_{2}}(T, N-1)= & T^{2} f_{d_{1}-1}(x) f_{d_{2}}(x) \\
& \left.+O\left(T\left(d_{1}+1+\delta_{i n}\right)^{-1-\frac{1}{c_{i n}(x)+\varepsilon}}\left(d_{2}+1+\delta_{i n}\right)^{-1-\frac{1}{c_{i n}(x)+\varepsilon}}\right)\right)+\theta_{5}, \\
D_{d_{1}, d_{2}-1}(T, N-1)= & T^{2} f_{d_{1}}(x) f_{d_{2}-1}(x) \\
& \left.+O\left(T\left(d_{1}+1+\delta_{i n}\right)^{-1-\frac{1}{c_{i n}(x)+\varepsilon}}\left(d_{2}+1+\delta_{i n}\right)^{-1-\frac{1}{c_{i n}(x)+\varepsilon}}\right)\right)+\theta_{6},
\end{aligned}
$$

where $\left|\theta_{4}\right| \leq C\left(T, d_{1}, d_{2}, x-\frac{1-x}{T}\right),\left|\theta_{5}\right| \leq C\left(T, d_{1}-1, d_{2}, x-\frac{1-x}{T}\right)$ if $d_{1} \geq 1$ and $\theta_{5}=0$ if $d_{1}=0,\left|\theta_{6}\right| \leq C\left(T, d_{1}, d_{2}-1, x-\frac{1-x}{T}\right)$ if $d_{2} \geq 1$ and $\theta_{6}=0$ if $d_{2}=0$.

Substitute these representations and results of Lemma 3.1 to (4.1) and expand:

$$
\begin{aligned}
& D_{d_{1}, d_{2}}(T+1, N) \\
= & T^{2} f_{d_{1}}(x) f_{d_{2}}(x)-(1-x+a x) \frac{d_{1}+d_{2}+2 \delta_{i n}}{1+\delta_{i n} x} T f_{d_{1}}(x) f_{d_{2}}(x) \\
& +(1-x+a x) \frac{d_{1}-1+\delta_{i n}}{1+\delta_{i n} x} T f_{d_{1}-1}(x) f_{d_{2}}(x) \\
& +(1-x+a x) \frac{d_{2}-1+\delta_{i n}}{1+\delta_{i n} x} T f_{d_{1}}(x) f_{d_{2}-1}(x) \\
& +\left[d_{1}=0\right] a x T f_{d_{2}}(x)+\left[d_{2}=0\right] a x T f_{d_{1}}(x) \\
& +\left[d_{1}=1\right](1-a) x T f_{d_{2}}(x)+\left[d_{2}=1\right](1-a) x T f_{d_{1}}(x) \\
& +O\left(\left(d_{1}+d_{2}+2\right)\left(d_{1}+1+\delta_{i n}\right)^{-1-\frac{1}{c_{i n}(x)+\varepsilon}}\left(d_{2}+1+\delta_{i n}\right)^{-1-\frac{1}{c_{i n}(x)+\varepsilon}}\right) \\
& +O\left(\frac{a x\left[d_{1}=0\right]+(1-a) x\left[d_{1}=1\right]}{1+\left(d_{2}+\delta_{i n}+1\right)^{1-\varepsilon} x^{2}}\right) \\
& +O\left(\frac{a x\left[d_{2}=0\right]+(1-a) x\left[d_{2}=1\right]}{1+\left(d_{1}+\delta_{i n}+1\right)^{1-\varepsilon} x^{2}}\right) \\
& +\theta_{1}(1-x)\left(1-\frac{d_{1}+d_{2}+2 \delta_{i n}}{T\left(1+\delta_{i n} x\right)+\delta_{i n} x+A_{i n}}\right)
\end{aligned}
$$




$$
\begin{aligned}
& +\theta_{2}(1-x) \frac{d_{1}-1+\delta_{i n}}{T\left(1+\delta_{i n} x\right)+\delta_{i n} x+A_{i n}} \\
& +\theta_{3}(1-x) \frac{d_{2}-1+\delta_{i n}}{T\left(1+\delta_{i n} x\right)+\delta_{i n} x+A_{i n}} \\
& +\theta_{4} x\left(1-\frac{a\left(d_{1}+d_{2}+2 \delta_{i n}\right)}{T\left(1+\delta_{i n} x\right)+\delta_{i n} x+A_{i n}-\delta_{i n}}\right) \\
& +\theta_{5} a x \frac{d_{1}-1+\delta_{i n}}{T\left(1+\delta_{i n} x\right)+\delta_{i n} x+A_{i n}-\delta_{i n}} \\
& +\theta_{6} a x \frac{d_{2}-1+\delta_{i n}}{T\left(1+\delta_{i n} x\right)+\delta_{i n} x+A_{i n}-\delta_{i n}} .
\end{aligned}
$$

The sum of terms without $\theta_{i}$ and $O(\cdot)$ equals $T^{2} f_{d_{1}}(x) f_{d_{2}}(x)+2 T f_{d_{1}}(x) f_{d_{2}}(x)$ from (3.2). Denote the sum of other terms as $\theta+f_{d_{1}}(x) f_{d_{2}}(x)$. Then, $D_{d_{1}, d_{2}}(T+1, N)=(T+$ $1)^{2} f_{d_{1}}(x) f_{d_{2}}(x)+\theta$, so we need to prove that $|\theta| \leq(T+1)\left(C_{1}\left(d_{1}, d_{2}, x\right)+C_{2}\left(d_{1}, d_{2}, x\right)\right)+$ $C_{3}\left(d_{1}, d_{2}, x\right)$.

From the Taylor formula and (4.5) it follows that

$$
\begin{aligned}
& \mid x C\left(T, d_{1}, d_{2}, x-\frac{1-x}{T}\right)+(1-x) C\left(T, d_{1}, d_{2}, x+\frac{x}{T}\right) \\
& -C\left(T, d_{1}, d_{2}, x\right) \mid \leq \frac{\varepsilon x(1-x) C\left(T, d_{1}, d_{2}, x\right)}{6 T\left(1+2 \delta_{\text {in }}+A_{\text {in }}\right)} .
\end{aligned}
$$

We know from (3.7) that $\left(d_{1}+\delta_{i n}\right) C_{3}\left(d_{1}, d_{2}, x\right)-\left(d_{1}-1+\delta_{i n}\right) C_{3}\left(d_{1}-1, d_{2}, x\right) \geq$ ${ }_{\varepsilon} C_{3}\left(d_{1}, d_{2}, x\right)$ if $d_{1} \geq 2$ and the same holds for $d_{1}=0$ and $d_{1}=1$ due to $C_{3}\left(0, d_{2}, x\right)=0$. Similarly, $\left(d+\delta_{i n}\right)\left(d+1+\delta_{i n}\right)^{-1+\varepsilon}-\left(d-1+\delta_{i n}\right)\left(d+\delta_{i n}\right)^{-1+\varepsilon}=\int_{d+\delta_{i n}}^{d+1+\delta_{i n}}((z-$ $\left.1) z^{-1+\varepsilon}\right)_{z}^{\prime} d z \geq 0$ if $d \geq 1$, so $\left(d_{1}+\delta_{i n}\right) C_{2}\left(d_{1}, d_{2}, x\right)-\left(d_{1}-1+\delta_{i n}\right) C_{2}\left(d_{1}-1, d_{2}, x\right) \geq 0$ (even for $\left.d_{1}=0\right)$. It follows from the definition of $\hat{f}_{d}(x)$ that $\left(d_{1}+\delta_{i n}\right) C_{1}\left(d_{1}, d_{2}, x\right)-\left(d_{1}-\right.$ $\left.1+\delta_{i n}\right) C_{1}\left(d_{1}-1, d_{2}, x\right) \geq-\frac{1}{c_{i n}(x)+\varepsilon} C_{1}\left(d_{1}, d_{2}, x\right)$ (with the equality if $d_{1} \geq 2$ ). Therefore,

$$
\begin{aligned}
& \left(d_{1}+\delta_{i n}\right) C\left(T, d_{1}, d_{2}, x\right)-\left(d_{1}+\delta_{i n}-1\right) C\left(T, d_{1}-1, d_{2}, x\right) \\
& \geq-\frac{T}{c_{i n}(x)+\varepsilon} C_{1}\left(d_{1}, d_{2}, x\right)+\varepsilon C_{3}\left(d_{1}, d_{2}, x\right), \\
& \left(d_{2}+\delta_{i n}\right) C\left(T, d_{1}, d_{2}, x\right)-\left(d_{2}+\delta_{i n}-1\right) C\left(T, d_{1}, d_{2}-1, x\right) \\
& \geq-\frac{T}{c_{i n}(x)+\varepsilon} C_{2}\left(d_{1}, d_{2}, x\right)+\varepsilon C_{3}\left(d_{1}, d_{2}, x\right), \\
& \left(d_{1}+d_{2}+2 \delta_{i n}\right) C\left(T, d_{1}, d_{2}, x\right)-\left(d_{1}+\delta_{i n}-1\right) C\left(T, d_{1}-1, d_{2}, x\right) \\
& \quad-\left(d_{2}+\delta_{i n}-1\right) C\left(T, d_{1}, d_{2}-1, x\right) \\
& \geq-\frac{T}{c_{i n}(x)+\varepsilon}\left(C_{1}\left(d_{1}, d_{2}, x\right)+C_{2}\left(d_{1}, d_{2}, x\right)\right)+2 \varepsilon C_{3}\left(d_{1}, d_{2}, x\right) .
\end{aligned}
$$


Thus,

$$
\begin{aligned}
|\theta| \leq & C\left(T, d_{1}, d_{2}, x\right)+\frac{\varepsilon x(1-x) C\left(T, d_{1}, d_{2}, x\right)}{6 T\left(1+2 \delta_{i n}+A_{i n}\right)} \\
& +\frac{T(1-x) \frac{1}{c_{i n}(x)+\varepsilon}\left(C_{1}\left(d_{1}, d_{2}, x+\frac{x}{T}\right)+C_{2}\left(d_{1}, d_{2}, x+\frac{x}{T}\right)\right)-(1-x) 2 \varepsilon C_{3}\left(d_{1}, d_{2}, x+\frac{x}{T}\right)}{T\left(1+\delta_{i n} x\right)+\delta_{i n} x+A_{i n}}-\operatorname{ax} 2 \varepsilon C_{3}\left(d_{1}, d_{2}, x-\frac{1-x}{T}\right) \\
& +\frac{\left.\operatorname{Tax} \frac{1}{c_{i n}(x)+\varepsilon}\left(C_{1}\left(d_{1}, d_{2}, x-\frac{1-x}{T}\right)+C_{2}\left(d_{1}, d_{2}, x-\frac{1-x}{T}\right)\right)-\delta_{i n} x\right)+\delta_{i n} x+A_{i n}-\delta_{i n}}{T\left(1+\delta_{i n}\right.} \\
& +O\left(\frac{\left(d_{1}+1+\delta_{i n}\right)^{-1-\frac{1}{c_{i n}(x)+\varepsilon}}}{1+\left(d_{2}+\delta_{i n}+1\right)^{1-\varepsilon} x^{2}}+\frac{\left(d_{2}+1+\delta_{i n}\right)^{-1-\frac{1}{c_{i n}(x)+\varepsilon}}}{1+\left(d_{1}+\delta_{i n}+1\right)^{1-\varepsilon} x^{2}}\right) .
\end{aligned}
$$

Apply bounds (4.3) and (4.4). Terms without $O(\cdot)$ become a linear combination of $C_{i}\left(d_{1}, d_{2}, x\right) ; C_{1}$ and $C_{2}$ have the coefficient that is not greater than $T+\frac{\varepsilon x(1-x)}{6\left(1+2 \delta_{i n}+A_{\text {in }}\right)}+$ $\frac{(1-x+a x)\left(1+\frac{\varepsilon}{3}\right)}{\left(1+\delta_{i n} x\right)\left(c_{i n}(x)+\varepsilon\right)} \leq T+\frac{\varepsilon}{6}+\frac{c_{i n}(x)\left(1+\frac{\varepsilon}{3}\right)}{c_{i n}(x)+\varepsilon} \leq T+\frac{\varepsilon}{6}+\frac{1+\frac{\varepsilon}{3}}{1+\varepsilon} \leq T+1-\frac{\varepsilon}{6} . C_{3}$ has the coefficient that is not greater than $1+\frac{\varepsilon x(1-x)}{6 T\left(1+2 \delta_{i n}+A_{i n}\right)}-\frac{(1-x+a x) 2 \varepsilon\left(1-\frac{\varepsilon}{3}\right)^{2}}{T\left(1+2 \delta_{i n}+A_{i n}\right)} \leq 1+\frac{\varepsilon(1-x)}{T\left(1+2 \delta_{i n}+A_{i n}\right)}\left(\frac{1}{6}-2\left(1-\frac{\varepsilon}{3}\right)^{2}\right) \leq 1$. It remains to select $C_{0}$ such that $\frac{\varepsilon}{6}\left(C_{1}\left(d_{1}, d_{2}, x\right)+C_{2}\left(d_{1}, d_{2}, x\right)\right) \geq O\left(\frac{\left(d_{1}+1+\delta_{i n}\right)^{-1-\frac{1}{c_{i n}(x)+\varepsilon}}}{1+\left(d_{2}+\delta_{i n}+1\right)^{1-\varepsilon} x^{2}}+\right.$ $\left.\frac{\left(d_{2}+1+\delta_{i n}\right)^{-1-\frac{1}{c_{i n}(x)+\varepsilon}}}{1+\left(d_{1}+\delta_{i n}+1\right)^{1-\varepsilon} x^{2}}\right)$.

Proof of Theorem 2.2 Without loss of generality assume $\varepsilon<1$. Let $x=\frac{N}{T}$. We have $\operatorname{Pr}\left(|x-(\alpha+\gamma)|>\sqrt{\frac{\ln T}{T}}\right) \rightarrow 0$ as $T \rightarrow \infty$; therefore, it is sufficient to show that

$\operatorname{Pr}\left(\left|n_{\text {in }}(G, d)-\overline{f_{d}} t\right|>\left(\sqrt{\overline{f_{d}}}+(d+1)^{-\frac{1}{2}+\varepsilon}\right) \ln t \mid G \in \mathcal{G}\left(T+t_{0}\right), \# G=N+n_{0}\right) \rightarrow 0$, uniformly, if $|x-(\alpha+\gamma)| \leq \sqrt{\frac{\ln T}{T}}$.

From now on, assume $|x-(\alpha+\gamma)| \leq \sqrt{\frac{\ln T}{T}}$. Then, $c_{i n}(x)=\Theta(1)$ and, similarly to (3.3), we have $f_{d}(x)=O\left((d+1)^{-1-\frac{1}{c_{i n}(x)}}\right)$ and $f_{d}^{\prime}(x)=O\left((d+1)^{-1-\frac{1}{c_{i n}(x)}} \ln (d+2)\right)$. Furthermore,

$$
\left|c_{i n}(x)-\bar{c}_{i n}\right|=O\left(|x-(\alpha+\gamma)| \max \left|c_{i n}^{\prime}(x)\right|\right)=O\left(\frac{\sqrt{\ln T}}{T}\right),
$$

$f_{d}(x)=O\left((d+1)^{-1-\frac{1}{\bar{c}_{i n}}+O\left(\sqrt{\frac{\ln T}{T}}\right)}\right)=O\left((d+1)^{-1-\frac{1}{\bar{c}_{i n}}}\right)$. Lemma 3.1 and the equality $\overline{f_{d}}=f_{d}(\alpha+\gamma)$ imply

$$
\begin{array}{r}
\mathbf{E}\left(n_{i n}(G, d) \mid G \in \mathcal{G}\left(T+t_{0}\right), \# G=N+n_{0}\right)=E_{d}(T, N)=T f_{d}(x)+O\left((d+1)^{-1+\varepsilon}\right) \\
=T \overline{f_{d}}+O\left(\sqrt{T \ln T}(d+1)^{-1-\frac{1}{\bar{c}_{i n}}} \ln (d+2)+(d+1)^{-1+\varepsilon}\right) .
\end{array}
$$

Lemmas 3.1 and 4.1 imply

$$
\begin{aligned}
D\left(n_{i n}(G, d) \mid G \in \mathcal{G}\left(T+t_{0}\right), \# G=N+n_{0}\right) & =D_{d, d}(T, N)+E_{d}(T, N)-E_{d}(T, N)^{2} \\
& =O\left(T(d+1)^{-1-\frac{1}{\bar{c}_{i n}}}+(d+1)^{-1+\varepsilon}\right) .
\end{aligned}
$$


If $\alpha=0$ and $d=0$, vertices with zero in-degree can come only from $G_{0}$, so $n_{i n}(G, 0)=$ $O(1)$ in this case, $\overline{f_{0}}=0$ and the theorem holds. Otherwise, $\overline{f_{d}}=\Theta\left((d+1)^{-1-\frac{1}{\bar{c}_{i n}}}\right)$ and if $t$ is sufficiently large, $\left|\mathbf{E} n_{i n}(G, d)-\overline{f_{d}} t\right| \leq \frac{1}{2}\left(\sqrt{\overline{f_{d}} t}+(d+1)^{-\frac{1}{2}+\varepsilon}\right) \ln t$,

$$
\begin{aligned}
\operatorname{Pr} & \left(\left|n_{\text {in }}(G, d)-\overline{f_{d}} t\right|>\left(\sqrt{\overline{f_{d}}}+(d+1)^{-\frac{1}{2}+\varepsilon}\right) \ln t \mid G \in \mathcal{G}\left(T+t_{0}\right), \# G=N+n_{0}\right) \\
& \leq \operatorname{Pr}\left(\left|n_{i n}(G, d)-\mathbf{E}\left(n_{i n}(G, d) \mid G \in \mathcal{G}\left(T+t_{0}\right), \# G=N+n_{0}\right)\right|\right. \\
& \left.>\frac{\ln t}{2}\left(\sqrt{\overline{f_{d}} t}+(d+1)^{-\frac{1}{2}+\varepsilon}\right) \mid \# G_{T}=N+n_{0}\right) \\
& \leq \frac{D\left(n_{i n}(G, d) \mid G \in \mathcal{G}\left(T+t_{0}\right), \# G=N+n_{0}\right)}{\frac{1}{4}\left(\sqrt{\overline{f_{d}}} t+(d+1)^{-\frac{1}{2}+\varepsilon}\right)^{2} \ln ^{2} t} \\
& =O\left(\frac{t(d+1)^{-1-\frac{1}{\bar{c}_{i n}}}+(d+1)^{-1+\varepsilon}}{\left(t(d+1)^{-1-\frac{1}{\bar{c}_{i n}}}+(d+1)^{-1+2 \varepsilon}\right) \ln ^{2} t}\right)=O\left(\frac{1}{\ln ^{2} t}\right)=o(1) .
\end{aligned}
$$

\section{EXPECTED NUMBER OF EDGES BETWEEN VERTICES WITH THE GIVEN DEGREE}

\subsection{Recurrent Equation}

Similar to the calculation of the number of vertices, let

$$
\begin{aligned}
E_{X}\left(T, N, d_{1}, d_{2}\right) & =\mathbf{E}\left(X\left(G, d_{1}, d_{2}\right) \mid G \in \mathcal{G}\left(T+t_{0}\right), \# G=N+n_{0}\right) \\
& =\sum_{\substack{i, j=1 \\
i \neq j}}^{N+n_{0}} \frac{\mathbf{E}\left(\left[\# G=N+n_{0}, d_{\text {out }}(G, i)=d_{1}, d_{i n}(G, j)=d_{2}\right] V(G, i, j)\right)}{\operatorname{Pr}\left(\# G=N+n_{0}\right)},
\end{aligned}
$$

where $V(G, i, j)$ is the number of edges from $i$ to $j$ in a graph $G$.

We start with a recurrent equation for $E_{X}$. Again, let $G_{T+1}$ be a random graph from $\mathcal{G}\left(T+t_{0}+1\right)$ with $N+n_{0}$ vertices.

- If $G_{T+1}$ is constructed from $G_{T} \in \mathcal{G}\left(T+t_{0}\right)$ using $(\dagger)$, then $N>0, G_{T}$ has $N+n_{0}-1$ vertices. Let $N+n_{0}$ be the new vertex and $w \in G_{T}$ be the target vertex of the new edge.

- If $i=N+n_{0}$ and $j=w$, then

$$
\left[d_{\text {out }}\left(G_{T+1}, i\right)=d_{1}, d_{i n}\left(G_{T+1}, j\right)=d_{2}\right] V\left(G_{T+1}, i, j\right)=\left[d_{1}=1, d_{i n}\left(G_{T}, j\right)=d_{2}-1\right] .
$$

- If $i=N+n_{0}$ and $j \neq w$, then

$$
\left[d_{\text {out }}\left(G_{T+1}, i\right)=d_{1}, d_{i n}\left(G_{T+1}, j\right)=d_{2}\right] V\left(G_{T+1}, i, j\right)=0 .
$$


- If $i \neq N+n_{0}$ and $j=w$, then

$$
\begin{aligned}
{\left[d_{\text {out }}\left(G_{T+1}, i\right)\right.} & \left.=d_{1}, d_{\text {in }}\left(G_{T+1}, j\right)=d_{2}\right] V\left(G_{T+1}, i, j\right) \\
& =\left[d_{\text {out }}\left(G_{T}, i\right)=d_{1}, d_{\text {in }}\left(G_{T}, j\right)=d_{2}-1\right] V\left(G_{T}, i, j\right) .
\end{aligned}
$$

- If $i \neq N+n_{0}$ and $j \neq w$, then

$$
\begin{aligned}
{\left[d_{\text {out }}\left(G_{T+1}, i\right)\right.} & \left.=d_{1}, d_{\text {in }}\left(G_{T+1}, j\right)=d_{2}\right] V\left(G_{T+1}, i, j\right) \\
& =\left[d_{\text {out }}\left(G_{T}, i\right)=d_{1}, d_{\text {in }}\left(G_{T}, j\right)=d_{2}\right] V\left(G_{T}, i, j\right) .
\end{aligned}
$$

Thus,

$$
\begin{aligned}
& \sum_{\substack{i, j=1 \\
i \neq j}}^{N+n_{0}}\left[d_{\text {out }}\left(G_{T+1}, i\right)=d_{1}, d_{i n}\left(G_{T+1}, j\right)=d_{2}\right] V\left(G_{T+1}, i, j\right) \\
& =\sum_{\substack{i, j=1 \\
i \neq j}}^{N+n_{0}-1}\left[d_{\text {out }}\left(G_{T}, i\right)=d_{1}, d_{\text {in }}\left(G_{T}, j\right)=d_{2}\right] V\left(G_{T}, i, j\right) \\
& \quad+\left[d_{1}=1, d_{\text {in }}\left(G_{T}, w\right)=d_{2}-1\right] \\
& \quad+\sum_{i=1}^{N+n_{0}-1}\left[d_{\text {out }}\left(G_{T}, i\right)=d_{1}, d_{\text {in }}\left(G_{T}, w\right)=d_{2}-1\right] V\left(G_{T}, i, w\right) \\
& \quad-\sum_{i=1}^{N+n_{0}-1}\left[d_{\text {out }}\left(G_{T}, i\right)=d_{1}, d_{\text {in }}\left(G_{T}, w\right)=d_{2}\right] V\left(G_{T}, i, w\right) .
\end{aligned}
$$

Recall that $w$ is selected with probability $\frac{d_{i n}\left(G_{T}, w\right)+\delta_{i n}}{T+\delta_{i n}(N-1)+A_{i n}}$.

$$
\begin{aligned}
\mathbf{E}( & X\left(G_{T+1}, d_{1}, d_{2} \mid G_{T},(\dagger)\right) \\
= & X\left(G_{T}, d_{1}, d_{2}\right)\left(1-\frac{d_{2}+\delta_{i n}}{T+\delta_{i n}(N-1)+A_{i n}}\right) \\
& +\frac{d_{2}-1+\delta_{i n}}{T+\delta_{i n}(N-1)+A_{i n}}\left[d_{1}=1\right] n_{i n}\left(G_{T}, d_{2}-1\right)+\frac{d_{2}-1+\delta_{i n}}{T+\delta_{i n}(N-1)+A_{i n}} \\
& \times X\left(G_{T}, d_{1}, d_{2}-1\right) .
\end{aligned}
$$

- The case $(\ddagger)$ is symmetrical to $(\dagger)$ with in- and out-degrees exchanged.

- If $G_{T+1}$ is constructed from $G_{T} \in \mathcal{G}\left(T+t_{0}\right)$ using (††), then $N<T+1, G_{T}$ has $N+n_{0}$ vertices. Let $v$ and $w$ be correspondingly the source and the target of the new edge. Then $d_{\text {out }}\left(G_{T+1}, i\right)=d_{\text {out }}\left(G_{T}, i\right)+[i=v], d_{\text {in }}\left(G_{T+1}, j\right)=d_{\text {in }}\left(G_{T}, j\right)+[j=w]$, $V\left(G_{T+1}, i, j\right)=V\left(G_{T}, i, j\right)+[i=v, j=w]$, so

$$
\begin{gathered}
\sum_{\substack{i, j=1 \\
i \neq j}}^{N+n_{0}}\left[d_{\text {out }}\left(G_{T+1}, i\right)=d_{1}, d_{i n}\left(G_{T+1}, j\right)=d_{2}\right] V\left(G_{T+1}, i, j\right) \\
\quad=\sum_{\substack{i, j=1 \\
i \neq j}}^{N+n_{0}}\left[d_{\text {out }}\left(G_{T}, i\right)=d_{1}, d_{\text {in }}\left(G_{T}, j\right)=d_{2}\right] V\left(G_{T}, i, j\right)
\end{gathered}
$$




$$
\begin{aligned}
& +\sum_{\substack{i=1 \\
i \neq w}}^{N+n_{0}}\left[d_{\text {out }}\left(G_{T}, i\right)=d_{1}\right] V\left(G_{T}, i, w\right)\left(\left[d_{\text {in }}\left(G_{T}, w\right)=d_{2}-1\right]-\left[d_{\text {in }}\left(G_{T}, w\right)=d_{2}\right]\right) \\
& +\sum_{\substack{j=1 \\
j \neq v}}^{N+n_{0}}\left[d_{\text {in }}\left(G_{T}, j\right)=d_{2}\right] V\left(G_{T}, v, j\right)\left(\left[d_{\text {out }}\left(G_{T}, v\right)=d_{1}-1\right]-\left[d_{\text {out }}\left(G_{T}, v\right)=d_{1}\right]\right) \\
& +\left[d_{\text {out }}\left(G_{T}, v\right)=d_{1}-1, d_{\text {in }}\left(G_{T}, w\right)=d_{2}-1, v \neq w\right]\left(V\left(G_{T}, v, w\right)+1\right) \\
& -\left[d_{\text {out }}\left(G_{T}, v\right)=d_{1}, d_{\text {in }}\left(G_{T}, w\right)=d_{2}-1, v \neq w\right] V\left(G_{T}, v, w\right) \\
& -\left[d_{\text {out }}\left(G_{T}, v\right)=d_{1}-1, d_{\text {in }}\left(G_{T}, w\right)=d_{2}, v \neq w\right] V\left(G_{T}, v, w\right) \\
& +\left[d_{\text {out }}\left(G_{T}, v\right)=d_{1}, d_{\text {in }}\left(G_{T}, w\right)=d_{2}, v \neq w\right] V\left(G_{T}, v, w\right) .
\end{aligned}
$$

Recall that $v$ and $w$ are selected independently with probabilities $\frac{d_{\text {out }}\left(G_{T}, v\right)+\delta_{\text {out }}}{T+\delta_{\text {out }} N+A_{\text {out }}}$ and $\frac{d_{i n}\left(G_{T}, w\right)+\delta_{i n}}{T+\delta_{i n} N+A_{\text {in }}}$, correspondingly.

$$
\begin{aligned}
& \mathbf{E}\left(X\left(G_{T+1}, d_{1}, d_{2} \mid G_{T},(\dagger \dagger)\right)\right. \\
&=X\left(G_{T}, d_{1}, d_{2}\right)\left(1-\frac{d_{2}+\delta_{\text {in }}}{T+\delta_{\text {in }} N+A_{\text {in }}}\right)\left(1-\frac{d_{1}+\delta_{\text {out }}}{T+\delta_{\text {out }} N+A_{\text {out }}}\right) \\
&+X\left(G_{T}, d_{1}, d_{2}-1\right) \frac{d_{2}-1+\delta_{\text {in }}}{T+\delta_{\text {in }} N+A_{\text {in }}}\left(1-\frac{d_{1}+\delta_{\text {out }}}{T+\delta_{\text {out }} N+A_{\text {out }}}\right) \\
&+X\left(G_{T}, d_{1}-1, d_{2}\right) \frac{d_{1}-1+\delta_{\text {out }}}{T+\delta_{\text {out }} N+A_{\text {out }}}\left(1-\frac{d_{2}+\delta_{\text {in }}}{T+\delta_{\text {in }} N+A_{\text {in }}}\right) \\
&+\left(\sum_{\substack{v, w=1 \\
v \neq w}}^{N+n_{0}}\left(d_{\text {out }}\left(G_{T}, v\right)=d_{1}-1, d_{\text {in }}\left(G_{T}, w\right)=d_{2}-1\right]+X\left(G_{T}, d_{1}-1, d_{2}-1\right)\right. \\
& \times \frac{d_{1}-1+\delta_{\text {out }}}{T+\delta_{\text {out }} N+A_{\text {out }}} \frac{d_{2}-1+\delta_{\text {in }}}{T+\delta_{\text {in }} N+A_{\text {in }}} .
\end{aligned}
$$

Finally, noting that $X\left(G_{T}, d_{1}-1, d_{2}-1\right)=O(T)$ and ignoring all terms that are $O_{d_{1}, d_{2}}(1 / T)$, we obtain

$$
\begin{aligned}
E_{X}(T & \left.+1, N, d_{1}, d_{2}\right) \\
= & {[N>0] \frac{\alpha}{\alpha+\gamma} \frac{N}{T+1}\left(E_{X}\left(T, N-1, d_{1}, d_{2}\right)\left(1-\frac{d_{2}+\delta_{i n}}{T+\delta_{i n}(N-1)+A_{i n}}\right)\right.} \\
& +\frac{d_{2}-1+\delta_{i n}}{T+\delta_{i n}(N-1)+A_{i n}}\left[d_{1}=1\right] E_{i n, d_{2}-1}(T, N-1) \\
& \left.+\frac{d_{2}-1+\delta_{i n}}{T+\delta_{i n}(N-1)+A_{i n}} E_{X}\left(T, N-1, d_{1}, d_{2}-1\right)\right)
\end{aligned}
$$




$$
\begin{aligned}
& +[N<T+1] \frac{T+1-N}{T+1}\left(E_{X}\left(T, N, d_{1}, d_{2}\right)\right. \\
& \times\left(1-\frac{d_{2}+\delta_{\text {in }}}{T+\delta_{\text {in }} N+A_{\text {in }}}-\frac{d_{1}+\delta_{\text {out }}}{T+\delta_{\text {out }} N+A_{\text {out }}}\right)+E_{X}\left(T, N, d_{1}, d_{2}-1\right) \\
& \times \frac{d_{2}-1+\delta_{\text {in }}}{T+\delta_{\text {in }} N+A_{\text {in }}}+E_{X}\left(T, N, d_{1}-1, d_{2}\right) \frac{d_{1}-1+\delta_{\text {out }}}{T+\delta_{\text {out }} N+A_{\text {out }}} \\
& \left.+E_{d_{1}-1, d_{2}-1}(T, N) \frac{d_{1}-1+\delta_{\text {out }}}{T+\delta_{\text {out }} N+A_{\text {out }}} \frac{d_{2}-1+\delta_{\text {in }}}{T+\delta_{\text {in }} N+A_{\text {in }}}\right) \\
& +[N>0] \frac{\gamma}{\alpha+\gamma} \frac{N}{T+1}\left(E_{X}\left(T, N-1, d_{1}, d_{2}\right)\left(1-\frac{d_{1}+\delta_{\text {out }}}{T+\delta_{\text {out }}(N-1)+A_{\text {out }}}\right)\right. \\
& +\frac{d_{1}-1+\delta_{\text {out }}}{T+\delta_{\text {out }}(N-1)+A_{\text {out }}}\left[d_{2}=1\right] E_{\text {out }}, d_{1}-1(T, N) \\
& \left.+\frac{d_{1}-1+\delta_{\text {out }}}{T+\delta_{\text {out }}(N-1)+A_{\text {out }}} E_{X}\left(T, N-1, d_{1}-1, d_{2}\right)\right)+O_{d_{1}, d_{2}}\left(\frac{1}{T}\right),
\end{aligned}
$$

where $E_{d_{1}, d_{2}}(T, N)=\mathbf{E}\left(\sum_{v \neq w}\left[d_{\text {out }}(G, v)=d_{1}, d_{i n}(G, w)=d_{2}\right] \mid G \in \mathcal{G}\left(T+t_{0}\right)\right.$, \#G= $\left.N+n_{0}\right), E_{\text {out }, d}(T, N)$, and $E_{i n, d}(T, N)$ are defined similarly to the proof of Theorem 2.1 as related to out- and in-degrees, correspondingly (thus, $E_{d}(T, N)$ from the proof of Theorem 2.2 is $E_{\text {in }, d}(T, N) ; E_{\text {out }, d}$ is the same with exchanged values $\left.\alpha \leftrightarrow \gamma, \delta_{\text {in }} \leftrightarrow \delta_{\text {out }}\right)$.

According to Lemma 3.1, $E_{\text {out }, d}(T, N)=T f_{\text {out }, d}\left(\frac{N}{T}\right)+O(1)$ and $E_{\text {in }, d}(T, N)=$ $T f_{\text {in, }}\left(\frac{N}{T}\right)+O(1)$, where

$$
\begin{aligned}
& f_{\text {in }, d}(x)=x \sum_{i=0}^{1}[d \geq i] p_{\text {in }, i} \frac{\Gamma\left(i+\delta_{\text {in }}+\frac{1}{c_{\text {in }}(x)}\right)}{c_{\text {in }}(x) \Gamma\left(i+\delta_{\text {in }}\right)} \frac{\Gamma\left(d+\delta_{\text {in }}\right)}{\Gamma\left(d+1+\delta_{\text {in }}+\frac{1}{c_{\text {in }}(x)}\right)}, \\
& f_{\text {out }, d}(x)=x \sum_{i=0}^{1}[d \geq i] p_{\text {out }, i} \frac{\Gamma\left(i+\delta_{\text {out }}+\frac{1}{c_{\text {out }}(x)}\right)}{c_{\text {out }}(x) \Gamma\left(i+\delta_{\text {out }}\right)} \frac{\Gamma\left(d+\delta_{\text {out }}\right)}{\Gamma\left(d+1+\delta_{\text {out }}+\frac{1}{c_{\text {out }}(x)}\right)},
\end{aligned}
$$

(these representations assume $\delta_{\text {in }}>0, \delta_{\text {out }}>0, c_{\text {in }}(x)>0$ and $c_{\text {out }}(x)>0$, but are more convenient to work with than the universal ones from Lemma 3.1).

The quantity $E_{d_{1}, d_{2}}(T, N)$ has the similar structure to $E_{X}\left(T, N, d_{1}, d_{2}\right)$, so the similar process gives a recurrent equation for $E_{d_{1}, d_{2}}(T, N)$ :

$$
\begin{aligned}
E_{d_{1}, d_{2}}(T+1, N) \\
=[N>0] \frac{\alpha}{\alpha+\gamma} \frac{N}{T+1}\left(E_{d_{1}, d_{2}}(T, N-1)\left(1-\frac{d_{2}+\delta_{\text {in }}}{T+\delta_{\text {in }}(N-1)+A_{\text {in }}}\right)\right. \\
\quad+\left[d_{1}=1\right] E_{\text {in, } d_{2}}(T, N-1)+\left[d_{2}=0\right] E_{\text {out }, d_{1}}(T, N-1) \\
\left.\quad+\frac{d_{2}-1+\delta_{\text {in }}}{T+\delta_{\text {in }}(N-1)+A_{\text {in }}} E_{d_{1}, d_{2}-1}(T, N-1)\right)+[N<T+1] \frac{T+1-N}{T+1} \\
\quad \times\left(E_{d_{1}, d_{2}}(T, N)\left(1-\frac{d_{2}+\delta_{\text {in }}}{T+\delta_{\text {in }} N+A_{\text {in }}}-\frac{d_{1}+\delta_{\text {out }}}{T+\delta_{\text {out }} N+A_{\text {out }}}\right)\right.
\end{aligned}
$$




$$
\begin{aligned}
& \left.+E_{d_{1}, d_{2}-1}(T, N) \frac{d_{2}-1+\delta_{\text {in }}}{T+\delta_{\text {in }} N+A_{\text {in }}}+E_{d_{1}-1, d_{2}}(T, N) \frac{d_{1}-1+\delta_{\text {out }}}{T+\delta_{\text {out }} N+A_{\text {out }}}\right) \\
& +[N>0] \frac{\gamma}{\alpha+\gamma} \frac{N}{T+1}\left(E_{d_{1}, d_{2}}(T, N-1)\left(1-\frac{d_{1}+\delta_{\text {out }}}{T+\delta_{\text {out }}(N-1)+A_{\text {out }}}\right)\right. \\
& +\left[d_{2}=1\right] E_{\text {out }, d_{1}}(T, N-1)+\left[d_{1}=0\right] E_{\text {in } d_{2}}(T, N-1) \\
& \left.+\frac{d_{1}-1+\delta_{\text {out }}}{T+\delta_{\text {out }}(N-1)+A_{\text {out }}} E_{d_{1}-1, d_{2}}(T, N-1)\right)+O_{d_{1}, d_{2}}(1)
\end{aligned}
$$

\subsection{Some Definite Integrals}

Define

$$
I_{1}\left(c_{1}, c_{2}, \xi_{1}, \xi_{2}, \xi_{3}, \xi_{4}\right)=\iint_{0 \leq v^{c_{1}} \leq w^{c_{2}} \leq 1} v^{\xi_{1}-1}(1-v)^{\xi_{2}} w^{\xi_{3}-1}(1-w)^{\xi_{4}} d v d w
$$

for $c_{1}>0, c_{2}>0, \xi_{1}>0, \xi_{2} \geq 0, \xi_{3}>0, \xi_{4} \geq 0$.

Lemma 5.1. The following recurrent equations for $I_{1}$ hold:

$$
\begin{gathered}
\left(c_{2}\left(\xi_{1}+\xi_{2}\right)+c_{1}\left(\xi_{3}+\xi_{4}\right)\right) I_{1}\left(c_{1}, c_{2}, \xi_{1}, \xi_{2}, \xi_{3}, \xi_{4}\right)=c_{1} \xi_{4} I_{1}\left(c_{1}, c_{2}, \xi_{1}, \xi_{2}, \xi_{3}, \xi_{4}-1\right) \\
+c_{2} \xi_{2} I_{1}\left(c_{1}, c_{2}, \xi_{1}, \xi_{2}-1, \xi_{3}, \xi_{4}\right) \\
\text { for } \xi_{2} \geq 1, \xi_{4} \geq 1 \\
\left(c_{2}\left(\xi_{1}+\xi_{2}\right)+c_{1} \xi_{3}\right) I_{1}\left(c_{1}, c_{2}, \xi_{1}, \xi_{2}, \xi_{3}, 0\right)=c_{1} \mathrm{~B}\left(\xi_{1}, \xi_{2}+1\right)+c_{2} \xi_{2} I_{1}\left(\xi_{1}, \xi_{2}-1, \xi_{3}, 0\right) \\
\text { for } \xi_{2} \geq 1 ; \\
\left(c_{2} \xi_{1}+c_{1}\left(\xi_{3}+\xi_{4}\right)\right) I_{1}\left(c_{1}, c_{2}, \xi_{1}, 0, \xi_{3}, \xi_{4}\right)=c_{1} \xi_{4} I_{1}\left(c_{1}, c_{2}, \xi_{1}, 0, \xi_{3}, \xi_{4}-1\right) \\
\quad \text { for } \xi_{4} \geq 1 ; \\
I_{1}\left(c_{1}, c_{2}, \xi_{1}, 0, \xi_{3}, 0\right)=\frac{c_{1}}{\xi_{1}\left(c_{2} \xi_{1}+c_{1} \xi_{3}\right)}
\end{gathered}
$$

\section{Proof.}

$$
I_{1}\left(c_{1}, c_{2}, \xi_{1}, \xi_{2}, \xi_{3}, \xi_{4}\right)=\int_{0}^{1} w^{\xi_{3}-1}(1-w)^{\xi_{4}}\left(\int_{0}^{w^{c_{2} / c_{1}}} v^{\xi_{1}-1}(1-v)^{\xi_{2}} d v\right) d w
$$

Let $\xi_{2} \geq 1$. Integrate by parts the inner integral, noting that $v=1-(1-v)$ :

$$
\begin{aligned}
& \xi_{1} \int_{0}^{w^{c_{2} / c_{1}}} v^{\xi_{1}-1}(1-v)^{\xi_{2}} d v \\
& \quad=\left.\left(v^{\xi_{1}}(1-v)^{\xi_{2}}\right)\right|_{0} ^{w^{c_{2} / c_{1}}}+\xi_{2} \int_{0}^{w^{c_{2} / c_{1}}} v^{\xi_{1}}(1-v)^{\xi_{2}-1} d v
\end{aligned}
$$




$$
\begin{aligned}
& =w^{\xi_{1} c_{2} / c_{1}}\left(1-w^{c_{2} / c_{1}}\right)^{\xi_{2}}+\xi_{2} \int_{0}^{w^{c_{2} / c_{1}}} v^{\xi_{1}-1}(1-v)^{\xi_{2}-1} d v-\xi_{2} \int_{0}^{w^{c_{2} / c_{1}}} v^{\xi_{1}-1}(1-v)^{\xi_{2}} d v \\
& \left(\xi_{1}+\xi_{2}\right) I_{1}\left(c_{1}, c_{2}, \xi_{1}, \xi_{2}, \xi_{3}, \xi_{4}\right) \\
& =\int_{0}^{1} w^{\xi_{1} c_{2} / c_{1}+\xi_{3}-1}(1-w)^{\xi_{4}}\left(1-w^{c_{2} / c_{1}}\right)^{\xi_{2}} d w+\xi_{2} I_{1}\left(c_{1}, c_{2}, \xi_{1}, \xi_{2}-1, \xi_{3}, \xi_{4}\right) .
\end{aligned}
$$

Let $\xi_{4} \geq 1$. Integrate by parts the outer integral:

$$
\begin{gathered}
\frac{d}{d w}\left((1-w)^{\xi_{4}}\left(\int_{0}^{w^{c_{2} / c_{1}}} v^{\xi_{1}-1}(1-v)^{\xi_{2}} d v\right)\right) \\
=-\xi_{4}(1-w)^{\xi_{4}-1}\left(\int_{0}^{w^{c_{2} / c_{1}}} v^{\xi_{1}-1}(1-v)^{\xi_{2}} d v\right) \\
+(1-w)^{\xi_{4}} \frac{c_{2}}{c_{1}} w^{c_{2} / c_{1}-1}\left(w^{c_{2} / c_{1}}\right)^{\xi_{1}-1}\left(1-w^{c_{2} / c_{1}}\right)^{\xi_{2}}, \\
\xi_{3} I_{1}\left(c_{1}, c_{2}, \xi_{1}, \xi_{2}, \xi_{3}, \xi_{4}\right)=\xi_{4}\left(I_{1}\left(c_{1}, c_{2}, \xi_{1}, \xi_{2}, \xi_{3}, \xi_{4}-1\right)-I_{1}\left(c_{1}, c_{2}, \xi_{1}, \xi_{2}, \xi_{3}, \xi_{4}\right)\right) \\
-\frac{c_{2}}{c_{1}} \int_{0}^{1} w^{\xi_{3}+\xi_{1} c_{2} / c_{1}-1}(1-w)^{\xi_{4}}\left(1-w^{c_{2} / c_{1}}\right)^{\xi_{2}} d w .
\end{gathered}
$$

The first equality of the lemma ( $\xi_{2} \geq 1$ and $\left.\xi_{4} \geq 1\right)$ follows from combining (5.3) and (5.4). For $\xi_{2} \geq 1$ and $\xi_{4}=0,(5.3)$ holds, but integration by parts yields an additional term instead of (5.4):

$$
\begin{aligned}
& \xi_{3} I_{1}\left(c_{1}, c_{2}, \xi_{1}, \xi_{2}, \xi_{3}, 0\right)=\int_{0}^{1} v^{\xi_{1}-1}(1-v)^{\xi_{2}} d v-\frac{c_{2}}{c_{1}} \int_{0}^{1} w^{\xi_{3}+\xi_{1} c_{2} / c_{1}-1}\left(1-w^{c_{2} / c_{1}}\right)^{\xi_{2}} d w \\
& =\mathrm{B}\left(\xi_{1}, \xi_{2}+1\right)-\frac{c_{2}}{c_{1}}\left(\left(\xi_{1}+\xi_{2}\right) I_{1}\left(c_{1}, c_{2}, \xi_{1}, \xi_{2}, \xi_{3}, 0\right)-\xi_{2} I_{1}\left(c_{1}, c_{2}, \xi_{1}, \xi_{2}-1, \xi_{3}, 0\right)\right) .
\end{aligned}
$$

Finally, the case $\xi_{2}=0$ is the same as $\xi_{2} \geq 1$, without the last term in (5.3).

Define

$$
\begin{aligned}
& I_{2}\left(c_{1}, c_{2}, \xi_{1}, \xi_{2}, \xi_{3}, \xi_{4}, \xi_{5}\right) \\
& \quad=\iint_{0 \leq v^{c_{1}} \leq w^{c_{2}} \leq 1} v^{\xi_{1}-1}(1-v)^{\xi_{2}} w^{\xi_{3}-1}(1-w)^{\xi_{4}}\left(\int_{w^{1 / c_{1}}}^{1} t^{\xi_{5}-1} d t\right) d v d w
\end{aligned}
$$

for $c_{1}>0, c_{2}>0, \xi_{1}>0, \xi_{2} \geq 0, \xi_{3}>0, \xi_{4} \geq 0, \xi_{3}+\xi_{5} / c_{i n}(x)>0$. (Note: $\xi_{5}$ can be zero or negative; in this case, the inner integral is not defined for $w=0$; the conditions ensure that the (improper) outer integral converges). 
Lemma 5.2. The following recurrent equations for $I_{2}$ hold:

$$
\begin{aligned}
& \left(c_{2}\left(\xi_{1}+\xi_{2}\right)+c_{1}\left(\xi_{3}+\xi_{4}\right)\right) I_{2}\left(c_{1}, c_{2}, \xi_{1}, \xi_{2}, \xi_{3}, \xi_{4}, \xi_{5}\right) \\
& =I_{1}\left(c_{1}, c_{2}, \xi_{1}, \xi_{2}, \xi_{3}+\frac{\xi_{5}}{c_{1}}, \xi_{4}\right)+c_{1} \xi_{4} I_{2}\left(c_{1}, c_{2}, \xi_{1}, \xi_{2}, \xi_{3}, \xi_{4}-1, \xi_{5}\right) \\
& \quad+c_{2} \xi_{2} I_{2}\left(c_{1}, c_{2}, \xi_{1}, \xi_{2}-1, \xi_{3}, \xi_{4}, \xi_{5}\right) \text { for } \xi_{2} \geq 1, \xi_{4} \geq 1 \\
& \left(c_{2}\left(\xi_{1}+\xi_{2}\right)+c_{1} \xi_{3}\right) I_{2}\left(c_{1}, c_{2}, \xi_{1}, \xi_{2}, \xi_{3}, 0, \xi_{5}\right) \\
& =I_{1}\left(c_{1}, c_{2}, \xi_{1}, \xi_{2}, \xi_{3}+\frac{\xi_{5}}{c_{1}}, 0\right)+c_{2} \xi_{2} I_{2}\left(c_{1}, c_{2}, \xi_{1}, \xi_{2}-1, \xi_{3}, 0, \xi_{5}\right) \\
& \quad \text { for } \xi_{2} \geq 1 \\
& \left(c_{2} \xi_{1}+c_{1}\left(\xi_{3}+\xi_{4}\right)\right) I_{2}\left(c_{1}, c_{2}, \xi_{1}, 0, \xi_{3}, \xi_{4}, \xi_{5}\right)=I_{1}\left(c_{1}, c_{2}, \xi_{1}, 0, \xi_{3}+\frac{\xi_{5}}{c_{1}}, \xi_{4}\right) \\
& +c_{1} \xi_{4} I_{2}\left(c_{1}, c_{2}, \xi_{1}, 0, \xi_{3}, \xi_{4}-1, \xi_{5}\right) \\
& \quad \text { for } \xi_{4} \geq 1 \\
& \left(c_{2} \xi_{1}+c_{1} \xi_{3}\right) I_{2}\left(c_{1}, c_{2}, \xi_{1}, 0, \xi_{3}, 0, \xi_{5}\right)=I_{1}\left(c_{1}, c_{2}, \xi_{1}, 0, \xi_{3}+\frac{\xi_{5}}{c_{1}}, 0\right)
\end{aligned}
$$

\section{Proof.}

$$
\begin{aligned}
& I_{2}\left(c_{1}, c_{2}, \xi_{1}, \xi_{2}, \xi_{3}, \xi_{4}, \xi_{5}\right) \\
& \quad=\int_{0}^{1} w^{\xi_{3}-1}(1-w)^{\xi_{4}}\left(\int_{0}^{w^{c_{2} / c_{1}}} v^{\xi_{1}-1}(1-v)^{\xi_{2}} d v\right)\left(\int_{w^{1 / c_{1}}}^{1} t^{\xi_{5}-1} d t\right) d w .
\end{aligned}
$$

Let $\xi_{2} \geq 1$ and $\xi_{4} \geq 1$. Similar to (5.3) from the previous lemma, integration by parts of $\int \ldots d v$ gives

$$
\begin{gathered}
\left(\xi_{1}+\xi_{2}\right) I_{2}\left(c_{1}, c_{2}, \xi_{1}, \xi_{2}, \xi_{3}, \xi_{4}, \xi_{5}\right)=\xi_{2} I_{2}\left(c_{1}, c_{2}, \xi_{1}, \xi_{2}-1, \xi_{3}, \xi_{4}, \xi_{5}\right) \\
\quad+\int_{0}^{1} w^{\xi_{1} c_{2} / c_{1}+\xi_{3}-1}(1-w)^{\xi_{4}}\left(1-w^{c_{2} / c_{1}}\right)^{\xi_{2}} \int_{w^{1 / c_{1}}}^{1} t^{\xi_{5}-1} d t d w .
\end{gathered}
$$

Integration by parts of $\int \ldots d w$ is similar to (5.5) from the previous lemma with an additional term from $\frac{d}{d w} \int_{w^{1 / c_{1}}}^{1} t^{\xi_{5}-1} d t=-\frac{1}{c_{1}} w^{\xi_{5} / c_{1}-1}$ :

$$
\begin{gathered}
\left(\xi_{3}+\xi_{4}\right) I_{2}\left(c_{1}, c_{2}, \xi_{1}, \xi_{2}, \xi_{3}, \xi_{4}, \xi_{5}\right)=\xi_{4} I_{2}\left(c_{1}, c_{2}, \xi_{1}, \xi_{2}, \xi_{3}, \xi_{4}-1, \xi_{5}\right) \\
-\frac{c_{2}}{c_{1}} \int_{0}^{1} w^{\xi_{3}+\xi_{1} c_{2} / c_{1}-1}(1-w)^{\xi_{4}}\left(1-w^{c_{2} / c_{1}}\right)^{\xi_{2}} \int_{w^{1 / c_{1}}}^{1} t^{\xi_{5}-1} d t d w \\
+\frac{1}{c_{1}} \int_{0}^{1} w^{\xi_{3}+\xi_{5} / c_{1}-1}(1-w)^{\xi_{4}}\left(\int_{0}^{w^{c_{2} / c_{1}}} v^{\xi_{1}-1}(1-v)^{\xi_{2}} d v\right) d w .
\end{gathered}
$$


The first equality of the lemma follows from combining (5.5) with (5.6) and recalling the definition of $I_{1}$. The case $\xi_{2}=0$ is similar to $(5.5)$ without the term $I_{2}\left(\ldots, \xi_{2}-1, \ldots\right)$; the case $\xi_{4}=0$ is similar to (5.6) without the term $I_{2}\left(\ldots, \xi_{4}-1, \ldots\right)$.

Define

$$
\begin{aligned}
& g_{1}\left(d_{1}, d_{2}\right) \\
& =\frac{\gamma}{\alpha+\gamma} \frac{x^{2}}{1+\delta_{\text {out }} x} \sum_{i=0}^{1}\left[d_{1} \geq i+1\right] \frac{p_{\text {out }, i}}{c_{\text {in }}(x) c_{\text {out }}(x)} \frac{\Gamma\left(d_{1}+\delta_{\text {out }}\right)}{\Gamma\left(d_{1}-i\right) \Gamma\left(\delta_{\text {out }}+i\right)} \frac{\Gamma\left(d_{2}+\delta_{\text {in }}\right)}{\Gamma\left(d_{2}\right) \Gamma\left(1+\delta_{\text {in }}\right)} \\
& \quad \times I_{1}\left(c_{\text {in }}(x), c_{\text {out }}(x), \delta_{\text {out }}+\frac{1}{c_{\text {out }}(x)}+i, d_{1}-i-1, \delta_{\text {in }}+\frac{c_{\text {out }}(x)}{c_{\text {in }}(x)}+1, d_{2}-1\right),
\end{aligned}
$$

$g_{2}\left(d_{1}, d_{2}\right)$

$$
\begin{aligned}
= & \frac{\alpha}{\alpha+\gamma} \frac{x^{2}}{1+\delta_{\text {in }} x} \sum_{i=0}^{1}\left[d_{2} \geq i+1\right] \frac{p_{\text {in }, i}}{c_{\text {in }}(x) c_{\text {out }}(x)} \frac{\Gamma\left(d_{1}+\delta_{\text {out }}\right)}{\Gamma\left(d_{1}\right) \Gamma\left(1+\delta_{\text {out }}\right)} \frac{\Gamma\left(d_{2}+\delta_{\text {in }}\right)}{\Gamma\left(d_{2}-i\right) \Gamma\left(\delta_{\text {in }}+i\right)} \\
& \times I_{1}\left(c_{\text {out }}(x), c_{\text {in }}(x), \delta_{\text {in }}+\frac{1}{c_{\text {in }}(x)}+i, d_{2}-i-1, \delta_{\text {out }}+\frac{c_{\text {in }}(x)}{c_{\text {out }}(x)}+1, d_{1}-1\right),
\end{aligned}
$$

$$
\begin{aligned}
g_{3}\left(d_{1}, d_{2}\right) & \\
= & \frac{x^{2}(1-x)}{\left(1+\delta_{\text {in }} x\right)\left(1+\delta_{\text {out }} x\right)} \sum_{i=0}^{1} \sum_{j=0}^{1}\left[d_{1} \geq i+1, d_{2} \geq j+1\right] \\
& \times \frac{p_{\text {out }, i} p_{\text {in }, j}}{c_{\text {in }}(x) c_{\text {out }}(x)} \frac{\Gamma\left(d_{1}+\delta_{\text {out }}\right)}{\Gamma\left(d_{1}-i\right) \Gamma\left(\delta_{\text {out }}+i\right)} \frac{\Gamma\left(d_{2}+\delta_{\text {in }}\right)}{\Gamma\left(d_{2}-j\right) \Gamma\left(\delta_{\text {in }}+j\right)} \\
& \times\left(I _ { 2 } \left(c_{\text {in }}(x), c_{\text {out }}(x), \delta_{\text {out }}+\frac{1}{c_{\text {out }}(x)}+i, d_{1}-i-1, \delta_{\text {in }}\right.\right. \\
& \left.+\frac{c_{\text {out }}(x)}{c_{\text {in }}(x)}+1+j, d_{2}-j-1,1-c_{\text {in }}(x)-c_{\text {out }}(x)\right) \\
& +I_{2}\left(c_{\text {out }}(x), c_{\text {in }}(x), \delta_{\text {in }}+\frac{1}{c_{\text {in }}(x)}+j, d_{2}-j-1, \delta_{\text {out }}\right. \\
& \left.\left.+\frac{c_{\text {in }}(x)}{c_{\text {out }}(x)}+1+i, d_{1}-i-1,1-c_{\text {in }}(x)-c_{\text {out }}(x)\right)\right)
\end{aligned}
$$

for $d_{1} \geq 1, d_{2} \geq 1$. Define $g_{i}\left(0, d_{2}\right)=g_{i}\left(d_{1}, 0\right)=0, i=1,2,3$.

Lemma 5.1 implies the following recurrent equations for $g_{1}, g_{2}$ and $d_{1} \geq 1, d_{2} \geq 1$ :

$$
\begin{aligned}
& \left(c_{\text {in }}(x)\left(d_{2}+\delta_{\text {in }}\right)+c_{\text {out }}(x)\left(d_{1}+\delta_{\text {out }}\right)+1\right) g_{1}\left(d_{1}, d_{2}\right) \\
& =c_{\text {in }}(x)\left(d_{2}-1+\delta_{\text {in }}\right) g_{1}\left(d_{1}, d_{2}-1\right)+c_{\text {out }}(x)\left(d_{1}-1+\delta_{\text {out }}\right) g_{1}\left(d_{1}-1, d_{2}\right) \\
& +\left[d_{2}=1\right] \frac{\gamma}{\alpha+\gamma} x \frac{d_{1}-1+\delta_{\text {out }}}{1+\delta_{\text {out }} x} f_{\text {out }, d_{1}-1}(x)
\end{aligned}
$$




$$
\begin{aligned}
\left(c_{\text {in }}(x)\left(d_{2}+\delta_{\text {in }}\right)+c_{\text {out }}(x)\left(d_{1}+\delta_{\text {out }}\right)\right. & +1) g_{2}\left(d_{1}, d_{2}\right) \\
=c_{\text {in }}(x)\left(d_{2}-1+\delta_{\text {in }}\right) g_{2}\left(d_{1}, d_{2}-1\right) & +c_{\text {out }}(x)\left(d_{1}-1+\delta_{\text {out }}\right) g_{2}\left(d_{1}-1, d_{2}\right) \\
+ & {\left[d_{1}=1\right] \frac{\alpha}{\alpha+\gamma} x \frac{d_{2}-1+\delta_{\text {in }}}{1+\delta_{\text {in }} x} f_{\text {in }, d_{2}-1}(x) . }
\end{aligned}
$$

Lemma 5.2 and the fact that

$$
\begin{aligned}
\mathrm{B}\left(\xi_{1}, \xi_{2}+1\right) \mathrm{B}\left(\xi_{3}, \xi_{4}+1\right) & =\iint_{0 \leq v^{c_{\text {in }}(x)} \leq w^{c_{\text {out }}(x)} \leq 1} v^{\xi_{1}-1}(1-v)^{\xi_{2}} w^{\xi_{3}-1}(1-w)^{\xi_{4}} d v d w \\
& +\iint_{0 \leq w^{c_{\text {out }}(x)} \leq v^{c_{\text {in }}(x)} \leq 1} v^{\xi_{1}-1}(1-v)^{\xi_{2}} w^{\xi_{3}-1}(1-w)^{\xi_{4}} d v d w \\
= & I_{1}\left(c_{\text {in }}(x), c_{\text {out }}(x), \xi_{1}, \xi_{2}, \xi_{3}, \xi_{4}\right)+I_{1}\left(c_{\text {out }}(x), c_{\text {in }}(x), \xi_{3}, \xi_{4}, \xi_{1}, \xi_{2}\right)
\end{aligned}
$$

imply that for $d_{1} \geq 1, d_{2} \geq 1$

$$
\begin{aligned}
\left(c_{\text {in }}(x)\left(d_{2}+\delta_{\text {in }}\right)+\right. & \left.c_{\text {out }}(x)\left(d_{1}+\delta_{\text {out }}\right)+1\right) g_{3}\left(d_{1}, d_{2}\right) \\
=c_{\text {in }}(x)\left(d_{2}-1\right. & \left.+\delta_{\text {in }}\right) g_{3}\left(d_{1}, d_{2}-1\right)+c_{\text {out }}(x)\left(d_{1}-1+\delta_{\text {out }}\right) g_{3}\left(d_{1}-1, d_{2}\right) \\
& +(1-x) \frac{d_{1}-1+\delta_{\text {out }}}{1+\delta_{\text {out }} x} \frac{d_{2}-1+\delta_{\text {in }}}{1+\delta_{\text {in }} x} f_{\text {out }, d_{1}-1}(x) f_{\text {in }, d_{2}-1}(x) .
\end{aligned}
$$

Finally, define

$$
g\left(d_{1}, d_{2}\right)=g\left(x, d_{1}, d_{2}\right)=g_{1}\left(d_{1}, d_{2}\right)+g_{2}\left(d_{1}, d_{2}\right)+g_{3}\left(d_{1}, d_{2}\right) .
$$

Then (5.7), (5.8), and (5.9) imply the recurrent equation

$$
\begin{gathered}
\left(c_{\text {in }}(x)\left(d_{2}+\delta_{\text {in }}\right)+c_{\text {out }}(x)\left(d_{1}+\delta_{\text {out }}\right)+1\right) g\left(d_{1}, d_{2}\right) \\
=c_{\text {in }}(x)\left(d_{2}-1+\delta_{\text {in }}\right) g\left(d_{1}, d_{2}-1\right)+c_{\text {out }}(x)\left(d_{1}-1+\delta_{\text {out }}\right) g\left(d_{1}-1, d_{2}\right) \\
+\left[d_{2}=1\right] \frac{\gamma}{\alpha+\gamma} x \frac{d_{1}-1+\delta_{\text {out }}}{1+\delta_{\text {out }} x} f_{\text {out }, d_{1}-1}(x)+\left[d_{1}=1\right] \frac{\alpha}{\alpha+\gamma} x \frac{d_{2}-1+\delta_{\text {in }}}{1+\delta_{\text {in }} x} f_{\text {in }, d_{2}-1}(x) \\
\quad+(1-x) \frac{d_{1}-1+\delta_{\text {out }}}{1+\delta_{\text {out }} x} \frac{d_{2}-1+\delta_{\text {in }}}{1+\delta_{\text {in }} x} f_{\text {out }, d_{1}-1}(x) f_{\text {in }, d_{2}-1}(x)
\end{gathered}
$$

Lemma 5.3. $\quad E_{X}\left(T, N, d_{1}, d_{2}\right)=g\left(\frac{N}{T}, d_{1}, d_{2}\right) T+O_{d_{1}, d_{2}}(1), \quad E_{d_{1}, d_{2}}(T, N)=$ $f_{\text {out }, d_{1}}\left(\frac{N}{T}\right) f_{\text {in, } d_{2}}\left(\frac{N}{T}\right) T^{2}+O_{d_{1}, d_{2}}(T)$.

Proof. The proof is essentially same as the proof of Lemma 3.1.

We use induction by $d_{1}+d_{2}$. Both sides of the first equation are zero when $d_{1}=0$ or $d_{2}=0$. Both sides of the second equation are zero when $d_{1}=-1$ or $d_{2}=-1$. The step of the induction is similar for both equations, we will show it for the first equation only. For $d_{1} \geq 1$ and $d_{2} \geq 1$ note that $g$ as a function in $x \in[0,1]$ is analytical, so $g\left(x, d_{1}, d_{2}\right)=O_{d_{1}, d_{2}}(1), g^{\prime}\left(x, d_{1}, d_{2}\right)=O_{d_{1}, d_{2}}(1), g^{\prime \prime}\left(x, d_{1}, d_{2}\right)=O_{d_{1}, d_{2}}(1)$ (we use 
derivatives with respect to $x$ ). For fixed $d_{1}$ and $d_{2}$, we use induction by $T$ to prove that

$$
\left|E_{X}\left(T, N, d_{1}, d_{2}\right)-g\left(\frac{N}{T}, d_{1}, d_{2}\right)\right| \leq C=C\left(d_{1}, d_{2}\right),
$$

where the constant $C\left(d_{1}, d_{2}\right)$ will be selected later, assuming that this inequality holds for $d_{1}-1, d_{2}$ and for $d_{1}, d_{2}-1$ with all $T$. Induction base $T \leq T_{0}\left(d_{1}, d_{2}\right)$ (where the value $T_{0}$ will be selected later) is trivial. Now assume that the bound for $T$ is proved and consider $T+1$. Let $0 \leq N \leq T+1$ and $x=\frac{N}{T+1}$. If $N \neq T+1$, then the inductive hypothesis and the Taylor formula imply that

$$
\begin{aligned}
E_{X}\left(T, N, d_{1}, d_{2}\right) & =T g\left(x+\frac{x}{T}, d_{1}, d_{2}\right)+\theta_{1} \\
& =T g\left(x, d_{1}, d_{2}\right)+x g^{\prime}\left(x, d_{1}, d_{2}\right)+\frac{x^{2}}{2 T} g^{\prime \prime}\left(\xi, d_{1}, d_{2}\right)+\theta_{1} \\
& =T g\left(x, d_{1}, d_{2}\right)+x g^{\prime}\left(x, d_{1}, d_{2}\right)+O_{d_{1}, d_{2}}\left(\frac{1}{T}\right)+\theta_{1},
\end{aligned}
$$

where $\left|\theta_{1}\right| \leq C$. Similarly, $E_{X}\left(T, N, d_{1}-1, d_{2}\right)=T g\left(x, d_{1}-1, d_{2}\right)+O_{d_{1}, d_{2}}(1)$ (even for $\left.d_{1}=1\right)$ and $E_{X}\left(T, N, d_{1}, d_{2}-1\right)=\operatorname{Tg}\left(x, d_{1}, d_{2}-1\right)+O_{d_{1}, d_{2}}(1)\left(\right.$ even for $\left.d_{2}=1\right)$. If $N \neq 0$, then we have, for the same reasons,

$$
\begin{aligned}
E_{X}\left(T, N-1, d_{1}, d_{2}\right) & =\operatorname{Tg}\left(x-\frac{1-x}{T}, d_{1}, d_{2}\right)+\theta_{2} \\
& =\operatorname{Tg}\left(x, d_{1}, d_{2}\right)-(1-x) g^{\prime}\left(x, d_{1}, d_{2}\right)+\frac{(1-x)^{2}}{2 T} g^{\prime \prime}\left(\xi, d_{1}, d_{2}\right)+\theta_{2} \\
& =\operatorname{Tg}\left(x, d_{1}, d_{2}\right)-(1-x) g^{\prime}\left(x, d_{1}, d_{2}\right)+O\left(\frac{1-x}{T}\right)+\theta_{2},
\end{aligned}
$$

where $\left|\theta_{2}\right| \leq C$. Similarly, $E_{X}\left(T, N-1, d_{1}-1, d_{2}\right)=T g\left(x, d_{1}-1, d_{2}\right)+O_{d_{1}, d_{2}}(1)$ and $E_{X}\left(T, N-1, d_{1}, d_{2}-1\right)=T g\left(x, d_{1}, d_{2}-1\right)+O_{d_{1}, d_{2}}(1)$.

Substitute these representations in (5.1). The sum of terms of order $T$ on the righthand side is $\operatorname{Tg}\left(x, d_{1}, d_{2}\right)$; terms of order 1 are exactly as in (5.11) and give $g\left(x, d_{1}, d_{2}\right)$ in total. Thus,

$$
\begin{aligned}
E_{X}\left(T+1, N, d_{1}, d_{2}\right) \\
=(T+1) g\left(\frac{N}{T+1}, d_{1}, d_{2}\right) \\
\quad+\theta_{1}(1-x)\left(1-\frac{d_{2}+\delta_{\text {in }}}{T\left(1+\delta_{\text {in }} x\right)+\delta_{\text {in }} x+A_{\text {in }}}-\frac{d_{1}+\delta_{\text {out }}}{T\left(1+\delta_{\text {out }} x\right)+\delta_{\text {out }} x+A_{\text {out }}}\right) \\
\quad+\theta_{2} x\left(1-\frac{\frac{\gamma}{\alpha+\gamma}\left(d_{2}+\delta_{\text {in }}\right)}{T\left(1+\delta_{\text {in }} x\right)+\delta_{\text {out }}(x-1)+A_{\text {in }}}-\frac{\frac{\gamma}{\alpha+\gamma}}{T\left(1+\delta_{\text {out }} x\right)+\delta_{\text {out }}(x-1)+A_{\text {out }}}\right) \\
\quad+O_{d_{1}, d_{2}}\left(\frac{1}{T}\right) .
\end{aligned}
$$


Select $T_{0}$ such that coefficients in $\theta_{i}$ are nonnegative for all $T \geq T_{0}$ and $x \in[0,1]$. Then, for any sufficiently large $C$ we have

$$
\begin{aligned}
& \left|E_{X}\left(T+1, N, d_{1}, d_{2}\right)-(T+1) g\left(\frac{N}{T+1}, d_{1}, d_{2}\right)\right| \leq C+O_{d_{1}, d_{2}}\left(\frac{1}{T}\right) \\
& -C\left(\frac{(1-x)\left(d_{2}+\delta_{\text {in }}\right)}{T\left(1+\delta_{\text {in }} x\right)+\delta_{\text {in }} x+A_{\text {in }}}+\frac{(1-x)\left(d_{1}+\delta_{\text {out }}\right)}{T\left(1+\delta_{\text {out }} x\right)+\delta_{\text {out }} x+A_{\text {out }}}\right. \\
& \left.\quad+\frac{\frac{\alpha}{\alpha+\gamma} x\left(d_{2}+\delta_{\text {in }}\right)}{T\left(1+\delta_{\text {in }} x\right)+\delta_{\text {in }}(x-1)+A_{\text {in }}}+\frac{\frac{\gamma}{\alpha+\gamma} x\left(d_{1}+\delta_{\text {out }}\right)}{T\left(1+\delta_{\text {out }} x\right)+\delta_{\text {out }}(x-1)+A_{\text {out }}}\right) \leq C .
\end{aligned}
$$

\subsection{Asymptotic Behavior}

\section{Lemma 5.4.}

$$
\kappa\left(c_{1}, c_{2}, r, x\right)=\Gamma\left(c_{1}\right) \Gamma\left(c_{2}\right)-\kappa\left(c_{2}, c_{1}, \frac{1}{r}, x^{-1 / r}\right) .
$$

\section{Proof.}

$$
\begin{aligned}
\int_{0}^{\infty} z^{c_{1}-1} e^{-z \tau^{r}} d z & =\left(\tau^{r}\right)^{-c_{1}} \int_{0}^{\infty}\left(z \tau^{r}\right)^{c_{1}-1} e^{-z \tau^{r}} d\left(z \tau^{r}\right)=\tau^{-c_{1} r} \Gamma\left(c_{1}\right) \\
\int_{0}^{\infty} \int_{0}^{\infty} z^{c_{1}-1} \tau^{c_{1} r+c_{2}-1} e^{-\tau-z \tau^{r}} d z d \tau & =\int_{0}^{\infty}\left(\tau^{r}\right)^{-c_{1}} \Gamma\left(c_{1}\right) \tau^{c_{1} r+c_{2}-1} e^{-\tau} d \tau=\Gamma\left(c_{1}\right) \Gamma\left(c_{2}\right) \\
\Gamma\left(c_{1}\right) \Gamma\left(c_{2}\right)-\kappa\left(c_{1}, c_{2}, r, x\right) & =\int_{x}^{\infty} d z \int_{0}^{\infty} z^{c_{1}-1} \tau^{c_{1} r+c_{2}-1} e^{-\tau-z \tau^{r}} d \tau
\end{aligned}
$$

Replace variables $\hat{z}=1 / z^{1 / r}, \hat{\tau}=z \tau^{r}: z=1 / \hat{z}^{r}, \tau=\hat{\tau}^{1 / r} \hat{z}, d z=-r \hat{z}^{-r-1} d \hat{z}, d \tau=$ $\frac{1}{r} \hat{z} \hat{\tau}^{1 / r-1} d \hat{\tau}$

$$
\Gamma\left(c_{1}\right) \Gamma\left(c_{2}\right)-\kappa\left(c_{1}, c_{2}, r, x\right)=\int_{0}^{x^{-1 / r}} d \hat{z} \int_{0}^{\infty} \hat{z}^{c_{2}-1} \hat{\tau}^{c_{1}+c_{2} / r-1} e^{-\hat{\tau}^{1 / r} \hat{z}-\hat{\tau}} d \hat{\tau}
$$

Lemma 5.5. Let $r>0, \xi_{1}>0, \xi_{3}>0$ be constants depending only on the model parameters. Then,

$$
I_{1}\left(c_{1}, c_{1} r, \xi_{1}, d_{1}, \xi_{3}, d_{2}\right)=d_{1}^{-\xi_{1}} d_{2}^{-\xi_{3}} \kappa\left(\xi_{1}, \xi_{3}, r, \frac{d_{1}}{d_{2}^{r}}\right)\left(1+O\left(\frac{1}{d_{2}^{\min (r, 1)}}\right)\right)
$$


Proof. Replace variables: let $v=w^{r} z$.

$$
\begin{aligned}
& \iint_{0 \leq v \leq w^{r} \leq 1} v^{\xi_{1}-1}(1-v)^{d_{1}} w^{\xi_{3}-1}(1-w)^{d_{2}} d v d w \\
& =\int_{0}^{1} \int_{0}^{1} w^{\xi_{1} r-r+\xi_{3}-1} z^{\xi_{1}-1}\left(1-w^{r} z\right)^{d_{1}}(1-w)^{d_{2}} w^{r} d z d w=\int_{0}^{1} z^{\xi_{1}-1} I_{z} d z
\end{aligned}
$$

where $I_{z}=\int_{0}^{1} w^{\xi_{1} r+\xi_{3}-1}(1-w)^{d_{2}}\left(1-w^{r} z\right)^{d_{1}} d w$.

Because $\ln (1+x) \leq x$ for any $x>-1$, we have $(1-w)^{d_{2}}=\exp \left(d_{2} \ln (1-w)\right) \leq$ $\exp \left(-d_{2} w\right)$ and $\left(1-w^{r} z\right)^{d_{1}} \leq \exp \left(-d_{1} w^{r} z\right)$, so

$$
I_{z} \leq \int_{0}^{\infty} w^{\xi_{1} r+\xi_{3}-1} e^{-d_{1} w^{r} z-d_{2} w} d w=d_{2}^{-\xi_{1} r-\xi_{3}} \int_{0}^{\infty} w^{\xi_{1} r+\xi_{3}-1} e^{-d_{1}\left(\frac{w}{d_{2}}\right)^{r} z-w} d w .
$$

If $r \geq 1$, let $\tau=\frac{w}{1-w}$, then $w=\frac{\tau}{1+\tau}, d w=\frac{d \tau}{(1+\tau)^{2}}$,

$$
I_{z}=\int_{0}^{\infty} \frac{\tau^{\xi_{1} r+\xi_{3}-1}}{(1+\tau)^{\xi_{1} r+\xi_{3}+1+d_{2}}}\left(1-z\left(\frac{\tau}{1+\tau}\right)^{r}\right)^{d_{1}} d \tau
$$

Comparing derivatives, it is easy to see that $(1+\tau)^{r} \geq 1+\tau^{r}$ for $r \geq 1$. Thus,

$$
\begin{aligned}
1 & -z\left(\frac{\tau}{1+\tau}\right)^{r} \geq 1-\frac{z \tau^{r}}{1+\tau^{r}} \geq 1-\frac{z \tau^{r}}{1+z \tau^{r}}=\frac{1}{1+z \tau^{r}} \geq \frac{1}{\exp \left(z \tau^{r}\right)} \\
I_{z} & \geq \int_{0}^{\infty} \tau^{\xi_{1} r+\xi_{3}-1} e^{-\left(\xi_{1} r+\xi_{3}+1+d_{2}\right) \tau-d_{1} z \tau^{r}} d \tau \\
& \geq\left(\xi_{1} r+\xi_{3}+1+d_{2}\right)^{-\xi_{1} r-\xi_{3}} \int_{0}^{\infty} \tau^{\xi_{1} r+\xi_{3}-1} e^{-\tau-d_{1} z\left(\frac{\tau}{d_{2}}\right)^{r}} d \tau \\
& =d_{2}^{-\xi_{1} r-\xi_{3}}\left(1+O\left(\frac{1}{d_{2}}\right)\right) \int_{0}^{\infty} \tau^{\xi_{1} r+\xi_{3}-1} e^{-\tau-\frac{d_{1}}{d_{2}} z \tau^{r}} d \tau .
\end{aligned}
$$

If $r \leq 1$, let $\tau=\frac{w}{\left(1-w^{r}\right)^{1 / r}}$, then $w=\frac{\tau}{\left(1+\tau^{r}\right)^{1 / r}}, w^{r-1} d w=\tau^{r-1} \frac{d \tau}{\left(1+\tau^{r}\right)^{2}}$,

$$
I_{z}=\int_{0}^{\infty} \frac{\tau^{\xi_{1} r+\xi_{3}-1}}{\left(1+\tau^{r}\right)^{\xi_{1}+\xi_{3} / r+1}}\left(1-\frac{\tau}{\left(1+\tau^{r}\right)^{1 / r}}\right)^{d_{2}}\left(1-\frac{z \tau^{r}}{1+\tau^{r}}\right)^{d_{1}} d \tau
$$

In this case, $1 / r \geq 1$, so $\left(1+\tau^{r}\right)^{1 / r} \geq 1+\left(\tau^{r}\right)^{1 / r}=1+\tau, 1-\frac{\tau}{\left(1+\tau^{r}\right)^{1 / r}} \geq \frac{1}{1+\tau} \geq \exp (-\tau)$, $1-\frac{z \tau^{r}}{1+\tau^{r}} \geq \frac{1}{1+z \tau^{r}} \geq \exp \left(-z \tau^{r}\right)$,

$$
I_{z} \geq \int_{0}^{\infty} \tau^{\xi_{1} r+\xi_{3}-1} e^{-d_{2} \tau-\left(z d_{1}+\xi_{1}+\xi_{3} / r+1\right) \tau^{r}} d \tau
$$


If $z d_{1} \geq d_{2}^{r}$, then $O\left(\frac{1}{z d_{1}}\right)=O\left(\frac{1}{d_{2}^{r}}\right)$, so

$$
\begin{aligned}
I_{z} & \geq\left(1+\frac{\xi_{1}+\xi_{3} / r+1}{z d_{1}}\right)^{-\xi_{1}-\xi_{3} / r} \int_{0}^{\infty} \tau^{\xi_{1} r+\xi_{3}-1} e^{-\frac{d_{2} \tau}{\left(1+\left(\xi_{1}+\xi_{3} / r+1\right) /\left(z d_{1}\right)\right)^{1 / r}}-z d_{1} \tau^{r}} d \tau \\
& \geq\left(1+O\left(\frac{1}{z d_{1}}\right)\right) \int_{0}^{\infty} \tau^{\xi_{1} r+\xi_{3}-1} e^{-d_{2} \tau-z d_{1} \tau^{r}} d \tau \\
& =\left(1+O\left(\frac{1}{d_{2}^{r}}\right)\right) d_{2}^{-\xi_{1} r-\xi_{3}} \int_{0}^{\infty} \tau^{\xi_{1} r+\xi_{3}-1} e^{-\tau-\frac{d_{1}}{d_{2}^{r}} z \tau^{r}} d \tau
\end{aligned}
$$

If $z d_{1}<d_{2}^{r}$, then $\exp \left(-\left(\xi_{1}+\xi_{3} / r+1\right) \frac{\tau^{r}}{d_{2}^{r}}\right) \geq 1-\left(\xi_{1}+\xi_{3} / r+1\right) \frac{\tau^{r}}{d_{2}^{r}}$,

$$
I_{z} \geq d_{2}^{-\xi_{1} r-\xi_{3}} \int_{0}^{\infty} \tau^{\xi_{1} r+\xi_{3}-1} e^{-\tau-z d_{1} \frac{\tau^{r}}{d_{2}^{r}}}\left(1-\left(\xi_{1}+\xi_{3} / r+1\right) \frac{\tau^{r}}{d_{2}^{r}}\right) d \tau,
$$

$\int_{0}^{\infty} \tau^{c} e^{-\tau-\tau^{r}\left(z d_{1} / d_{2}^{r}\right)} d \tau=\Theta(1)$ for $c=\xi_{1} r+\xi_{3}-1$ and for $c=\xi_{1} r+\xi_{3}+r-1$, so

$$
I_{z} \geq d_{2}^{-\xi_{1} r-\xi_{3}}\left(1+O\left(\frac{1}{d_{2}^{r}}\right)\right) \int_{0}^{\infty} \tau^{\xi_{1} r+\xi_{3}-1} e^{-\tau-z d_{1} \frac{\tau^{r}}{d_{2}^{r}}} d \tau
$$

Integration by $z$ completes the proof.

Lemma 5.6. Let $r>0, \xi_{1}>0, \xi_{3}>0$ be constants depending only on the model parameters. Then,

$$
I_{1}\left(c_{1}, c_{1} r, \xi_{1}, d_{1}, \xi_{3}, d_{2}\right)=d_{1}^{-\xi_{1}} d_{2}^{-\xi_{3}} \kappa\left(\xi_{1}, \xi_{3}, r, \frac{d_{1}}{d_{2}^{r}}\right)\left(1+O\left(\frac{1}{d_{1}}\right)+O\left(\frac{1}{d_{2}}\right)\right)
$$

Proof. If $r \geq 1$ or $d_{1} \leq C d_{2}^{r}$, the statement follows immediately from the previous lemma. Assume $r<1$ and $d_{1}>C d_{2}^{r}$, where the constant $C$ will be selected later.

$$
\begin{aligned}
& \iint_{0 \leq v \leq w^{r} \leq 1} v^{\xi_{1}-1}(1-v)^{d_{1}} w^{\xi_{3}-1}(1-w)^{d_{2}} d v d w \\
& =\mathrm{B}\left(\xi_{1}, d_{1}+1\right) \mathrm{B}\left(\xi_{3}, d_{2}+1\right)-\iint_{0 \leq w^{r} \leq v \leq 1} v^{\xi_{1}-1}(1-v)^{d_{1}} w^{\xi_{3}-1}(1-w)^{d_{2}} d v d w \\
& =\Gamma\left(\xi_{1}\right) \Gamma\left(\xi_{3}\right) d_{1}^{-\xi_{1}} d_{2}^{-\xi_{3}}\left(1+O\left(\frac{1}{d_{1}}\right)+O\left(\frac{1}{d_{2}}\right)\right) \\
& \quad-\iint_{0 \leq w \leq v^{1 / r} \leq 1} w^{\xi_{3}-1}(1-w)^{d_{2}} v^{\xi_{1}-1}(1-v)^{d_{1}} d v d w \\
& =\Gamma\left(\xi_{1}\right) \Gamma\left(\xi_{3}\right) d_{1}^{-\xi_{1}} d_{2}^{-\xi_{3}}\left(1+O\left(\frac{1}{d_{1}}\right)+O\left(\frac{1}{d_{2}}\right)\right) \\
& \quad-d_{1}^{-\xi_{1}} d_{2}^{-\xi_{3}} \kappa\left(\xi_{3}, \xi_{1}, \frac{1}{r}, \frac{d_{2}}{d_{1}^{1 / r}}\right)\left(1+O\left(\frac{1}{d_{1}}\right)\right)
\end{aligned}
$$


Lemma 5.4 implies that

$$
\Gamma\left(\xi_{1}\right) \Gamma\left(\xi_{3}\right)-\kappa\left(\xi_{3}, \xi_{1}, \frac{1}{r}, \frac{d_{2}}{d_{1}^{1 / r}}\right)=\kappa\left(\xi_{1}, \xi_{3}, r, \frac{d_{1}}{d_{2}^{r}}\right) .
$$

However, $\kappa\left(c_{1}, c_{2}, r, x\right)=x^{c_{1}} \int_{0}^{1} z^{c_{1}-1} d z \int_{0}^{\infty} \tau^{c_{1} r+c_{2}-1} e^{-\tau-x z \tau^{r}} d \tau \leq x^{c_{1}} \frac{\Gamma\left(c_{1} r+c_{2}\right)}{\xi_{3}}$, so

$$
\kappa\left(\xi_{3}, \xi_{1}, \frac{1}{r}, \frac{d_{2}}{d_{1}^{1 / r}}\right) \leq C^{-\xi_{3} / r} \frac{\Gamma\left(\xi_{1}+\xi_{3} / r\right)}{\xi_{3}} \leq \frac{1}{2} \Gamma\left(\xi_{1}\right) \Gamma\left(\xi_{3}\right)
$$

if $C$ is sufficiently large, so, $O(1)=O\left(\Gamma\left(\xi_{1}\right) \Gamma\left(\xi_{3}\right)-\kappa\left(\xi_{3}, \xi_{1}, \frac{1}{r}, \frac{d_{2}}{d_{1}^{1 / r}}\right)\right)$.

Lemma 5.7. Let $c_{1}>0, c_{2}>0, \xi_{1}>0, \xi_{3}>0, \xi_{5}>-\xi_{3} c_{i n}(x)$ be constants depending only on the model parameters. Let $d_{1} \geq 1, d_{2} \geq 1$. Then,

$$
\begin{gathered}
I_{1}\left(c_{1}, c_{2}, \xi_{1}, d_{1} \pm 1, \xi_{3}, d_{2}\right)=I_{1}\left(c_{1}, c_{2}, \xi_{1}, d_{1}, \xi_{3}, d_{2}\right)\left(1+O\left(\frac{1}{d_{1}}\right)\right) \\
I_{1}\left(c_{1}, c_{2}, \xi_{1}, d_{1}, \xi_{3}, d_{2} \pm 1\right)=I_{1}\left(c_{1}, c_{2}, \xi_{1}, d_{1}, \xi_{3}, d_{2}\right)\left(1+O\left(\frac{1}{d_{2}}\right)\right) \\
I_{2}\left(c_{1}, c_{2}, \xi_{1}, d_{1} \pm 1, \xi_{3}, d_{2}, \xi_{5}\right)=I_{2}\left(c_{1}, c_{2}, \xi_{1}, d_{1}, \xi_{3}, d_{2}, \xi_{5}\right)\left(1+O\left(\frac{1}{d_{1}}\right)\right) \\
I_{2}\left(c_{1}, c_{2}, \xi_{1}, d_{1}, \xi_{3}, d_{2} \pm 1, \xi_{5}\right)=I_{2}\left(c_{1}, c_{2}, \xi_{1}, d_{1}, \xi_{3}, d_{2}, \xi_{5}\right)\left(1+O\left(\frac{1}{d_{2}}\right)\right) .
\end{gathered}
$$

Proof. It is easy to see from the definition of $I_{1}$ that $I_{1}\left(c_{1}, c_{1} r, \xi_{1}, d_{1}, \xi_{3}, d_{2}\right) \leq$ $I_{1}\left(c_{1}, c_{1} r, \xi_{1}, d_{1}-1, \xi_{3}, d_{2}\right)$ and $I_{1}\left(c_{1}, c_{1} r, \xi_{1}, d_{1}, \xi_{3}, d_{2}\right) \leq I_{1}\left(c_{1}, c_{1} r, \xi_{1}, d_{1}, \xi_{3}, d_{2}-1\right)$. However, Lemma 5.1 implies

$$
\begin{gathered}
\left(c_{2}\left(\xi_{1}+d_{1}\right)+c_{1}\left(\xi_{3}+d_{2}\right)\right) I_{1}\left(c_{1}, c_{2}, \xi_{1}, d_{1}, \xi_{3}, d_{2}\right) \\
=c_{1} d_{2} I_{1}\left(c_{1}, c_{2}, \xi_{1}, d_{1}, \xi_{3}, d_{2}-1\right)+c_{2} d_{1} I_{1}\left(c_{1}, c_{2}, \xi_{1}, d_{1}-1, \xi_{3}, d_{2}\right) \\
\geq c_{1} d_{2} I_{1}\left(c_{1}, c_{2}, \xi_{1}, d_{1}, \xi_{3}, d_{2}\right)+c_{2} d_{1} I_{1}\left(c_{1}, c_{2}, \xi_{1}, d_{1}-1, \xi_{3}, d_{2}\right), \\
\left(c_{2} \xi_{1}+c_{1} \xi_{3}+c_{2} d_{1}\right) I_{1}\left(c_{1}, c_{2}, \xi_{1}, d_{1}, \xi_{3}, d_{2}\right) \geq c_{2} d_{1} I_{1}\left(c_{1}, c_{2}, \xi_{1}, d_{1}-1, \xi_{3}, d_{2}\right), \\
I_{1}\left(c_{1}, c_{2}, \xi_{1}, d_{1}-1, \xi_{3}, d_{2}\right) \leq\left(1+\frac{c_{2} \xi_{1}+c_{1} \xi_{3}}{c_{2} d_{1}}\right) I_{1}\left(c_{1}, c_{2}, \xi_{1}, d_{1}, \xi_{3}, d_{2}\right), \\
I_{1}\left(c_{1}, c_{2}, \xi_{1}, d_{1}, \xi_{3}, d_{2}\right) \geq\left(1-\frac{c_{2} \xi_{1}+c_{1} \xi_{3}}{c_{2} d_{1}}\right) I_{1}\left(c_{1}, c_{2}, \xi_{1}, d_{1}-1, \xi_{3}, d_{2}\right) .
\end{gathered}
$$

This proves the first statement of the lemma, other are analogous (for $I_{2}$, use Lemma 5.2 instead of Lemma 5.1 and note that $I_{1} \geq 0$ ). 
Lemma 5.8. Let $c_{1}>0, r>0, \xi_{1}>0, \xi_{3}>0$ be constants depending only on the model parameters. If $\xi_{5} \neq 0$, then

$$
\begin{aligned}
& I_{2}\left(c_{1}, c_{1} r, \xi_{1}, d_{1}, \xi_{3}, d_{2}, \xi_{5}\right)=\frac{1}{\xi_{5}} d_{1}^{-\xi_{1}}\left(d_{2}^{-\xi_{3}} \kappa\left(\xi_{1}, \xi_{3}, r, \frac{d_{1}}{d_{2}^{r}}\right)\left(1+O\left(\frac{1}{d_{1}}+\frac{1}{d_{2}}\right)\right)\right. \\
&\left.-d_{2}^{-\xi_{3}-\frac{\xi_{5}}{c_{1}}} \kappa\left(\xi_{1}, \xi_{3}+\frac{\xi_{5}}{c_{1}}, r, \frac{d_{1}}{d_{2}^{r}}\right)\left(1+O\left(\frac{1}{d_{1}}+\frac{1}{d_{2}}\right)\right)\right) .
\end{aligned}
$$

If $\xi_{5}=0$, then

$$
\begin{gathered}
I_{2}\left(c_{1}, c_{1} r, \xi_{1}, d_{1}, \xi_{3}, d_{2}, 0\right)=\frac{1}{c_{1}} d_{1}^{-\xi_{1}} d_{2}^{-\xi_{3}}\left(\kappa\left(\xi_{1}, \xi_{3}, r, \frac{d_{1}}{d_{2}^{r}}\right) \ln d_{2}\right. \\
\left.-\frac{\partial}{\partial \xi_{3}} \kappa\left(\xi_{1}, \xi_{3}, r, \frac{d_{1}}{d_{2}^{r}}\right)\right)+O\left(d_{1}^{-\xi_{1}} d_{2}^{-\xi_{3}}\left(\frac{\ln d_{2}}{d_{1}}+\frac{1}{d_{2}}\right)\right) .
\end{gathered}
$$

Proof. The first statement follows from the equality

$I_{2}\left(c_{1}, c_{1} r, \xi_{1}, d_{1}, \xi_{3}, d_{2}, \xi_{5}\right)=\frac{I_{1}\left(c_{1}, c_{1} r, \xi_{1}, d_{1}, \xi_{3}, d_{2}\right)-I_{1}\left(c_{1}, c_{1} r, \xi_{1}, d_{1}, \xi_{3}+\xi_{5} / c_{1}, d_{2}\right)}{\xi_{5}}$, and Lemma 5.6.

The case $\xi_{5}=0$ is special.

$I_{2}\left(c_{1}, c_{1} r, \xi_{1}, d_{1}, \xi_{3}, d_{2}, 0\right)=\frac{1}{c_{1}} \iint_{0 \leq v^{c_{1}} \leq w^{c_{1} r} \leq 1} v^{\xi_{1}-1}(1-v)^{d_{1}} w^{\xi_{3}-1}(1-w)^{d_{2}}(-\ln w) d v d w$

For the upper bound, note that $1-v \leq \exp (-v), 1-w \leq \exp (-w)$ :

$$
\begin{aligned}
c_{1} I_{2}\left(c_{1}, c_{1} r, \xi_{1}, d_{1}, \xi_{3}, d_{2}, 0\right) \leq & \iint_{0 \leq v^{c_{1}} \leq w^{c_{1} r} \leq 1} v^{\xi_{1}-1} w^{\xi_{3}-1} e^{-d_{1} v-d_{2} w}(-\ln w) d v d w \\
\leq & \iint_{0 \leq v \leq w^{r}<\infty} v^{\xi_{1}-1} w^{\xi_{3}-1} e^{-d_{1} v-d_{2} w}(-\ln w) d v d w \\
& +\iint_{v \geq 0, w \geq 1} v^{\xi_{1}-1} w^{\xi_{3}-1} e^{-d_{1} v-d_{2} w} \ln w d v d w \\
\int_{0}^{\infty} v^{\xi_{1}-1} e^{-d_{1} v} d v= & d_{1}^{-\xi_{1}} \Gamma\left(\xi_{1}\right), \int_{1}^{\infty} w^{\xi_{3}-1} e^{-d_{2} w} \ln w d w \\
\leq & e^{-d_{2}} \int_{1}^{\infty} w^{\xi_{3}-1} e^{-(w-1)} \ln w d w=O\left(e^{-d_{2}}\right)
\end{aligned}
$$

so

$$
\begin{aligned}
c_{1} I_{2}\left(c_{1}, c_{1} r, \xi_{1}, d_{1}, \xi_{3}, d_{2}, 0\right) \leq & \iint_{0 \leq v \leq w^{r}<\infty} v^{\xi_{1}-1} w^{\xi_{3}-1} e^{-d_{1} v-d_{2} w}(-\ln w) d v d w \\
& +O\left(d_{1}^{-\xi_{1}} e^{-d_{2}}\right) .
\end{aligned}
$$


For the lower bound, note that

$$
\begin{aligned}
& c_{1} I_{2}\left(c_{1}, c_{1} r, \xi_{1}, d_{1}, \xi_{3}, d_{2}, 0\right) \\
& =\left(\int_{0}^{1} v^{\xi_{1}-1}(1-v)^{d_{1}} d v\right)\left(\int_{0}^{1} w^{\xi_{3}-1}(1-w)^{d_{2}}(-\ln w) d w\right) \\
& \quad-\iint_{0 \leq w \leq v^{1 / r} \leq 1} v^{\xi_{1}-1}(1-v)^{d_{1}} w^{\xi_{3}-1}(1-w)^{d_{2}}(-\ln w) d v d w ;
\end{aligned}
$$

$\int_{0}^{1} v^{\xi_{1}-1}(1-v)^{d_{1}} d v=\mathrm{B}\left(\xi_{1}, d_{1}+1\right)=\Gamma\left(\xi_{1}\right) d_{1}^{-\xi_{1}}\left(1+O\left(\frac{1}{d_{1}}\right)\right)$; according to [1, 6.3.18], we have $\frac{\Gamma^{\prime}(x)}{\Gamma(x)}=\ln x+O\left(\frac{1}{x}\right)$, so

$$
\begin{aligned}
& \int_{0}^{1} w^{\xi_{3}-1}(1-w)^{d_{2}}(-\ln w) d w=-\frac{\partial}{\partial \xi_{3}} \mathrm{~B}\left(\xi_{3}, d_{2}+1\right) \\
&=-\frac{\Gamma^{\prime}\left(\xi_{3}\right) \Gamma\left(d_{2}+1\right)}{\Gamma\left(\xi_{3}+d_{2}+1\right)}+\frac{\Gamma\left(\xi_{3}\right) \Gamma\left(d_{2}+1\right) \Gamma^{\prime}\left(\xi_{3}+d_{2}+1\right)}{\Gamma\left(\xi_{3}+d_{2}+1\right)^{2}} \\
&= d_{2}^{-\xi_{3}}\left(-\Gamma^{\prime}\left(\xi_{3}\right)+\Gamma\left(\xi_{3}\right) \ln d_{2}+O\left(\frac{1}{d_{2}}\right)\right) \\
& \iint_{0 \leq w \leq v^{1 / r} \leq 1} v^{\xi_{1}-1}(1-v)^{d_{1}} w^{\xi_{3}-1}(1-w)^{d_{2}}(-\ln w) d v d w \\
& \leq \iint_{0 \leq w \leq v^{1 / r} \leq 1} v^{\xi_{1}-1} w^{\xi_{3}-1} e^{-d_{1} v-d_{2} w}(-\ln w) d v d w \\
& \leq \iint_{0 \leq w \leq v^{1 / r}<\infty} v^{\xi_{1}-1} w^{\xi_{3}-1} e^{-d_{1} v-d_{2} w}(-\ln w) d v d w \\
& \quad+\iint_{1 \leq w \leq v^{1 / r}<\infty} v^{\xi_{1}-1} w^{\xi_{3}-1} e^{-d_{1} v-d_{2} w} \ln w d v d w \\
&=\iint_{0 \leq w \leq v^{1 / r}<\infty} v^{\xi_{1}-1} w^{\xi_{3}-1} e^{-d_{1} v-d_{2} w}(-\ln w) d v d w+O\left(e^{-d_{1}-d_{2}}\right) .
\end{aligned}
$$

Since $\int_{0}^{\infty} v^{\xi_{1}-1} e^{-d_{1} v} d v=\Gamma\left(\xi_{1}\right) d_{1}^{-\xi_{1}}$ and

$$
\int_{0}^{\infty} w^{\xi_{3}-1} e^{-d_{2} w}(-\ln w) d w=-\frac{\partial}{\partial \xi_{3}}\left(\Gamma\left(\xi_{3}\right) d_{2}^{-\xi_{3}}\right)=d_{2}^{-\xi_{3}}\left(-\Gamma^{\prime}\left(\xi_{3}\right)+\Gamma\left(\xi_{3}\right) \ln d_{2}\right),
$$

we have

$$
\begin{aligned}
c_{1} I_{2}\left(c_{1}, c_{1} r, \xi_{1}, d_{1}, \xi_{3}, d_{2}, 0\right) \geq & d_{1}^{-\xi_{1}} d_{2}^{-\xi_{3}} O\left(\frac{\ln d_{2}}{d_{1}}+\frac{1}{d_{2}}\right) \\
& +\iint_{0 \leq v \leq w^{r}<\infty} v^{\xi_{1}-1} w^{\xi_{3}-1} e^{-d_{1} v-d_{2} w}(-\ln w) d v d w \\
& +O\left(e^{-d_{1}-d_{2}}\right) .
\end{aligned}
$$


Combine lower and upper bound:

$$
\begin{aligned}
c_{1} I_{2}\left(c_{1}, c_{1} r, \xi_{1}, d_{1}, \xi_{3}, d_{2}, 0\right)= & \iint_{0 \leq v \leq w^{r}<\infty} v^{\xi_{1}-1} w^{\xi_{3}-1} e^{-d_{1} v-d_{2} w}(-\ln w) d v d w \\
& +d_{1}^{-\xi_{1}} d_{2}^{-\xi_{3}} O\left(\frac{\ln d_{2}}{d_{1}}+\frac{1}{d_{2}}\right) .
\end{aligned}
$$

Replace variables: let $v=w^{r} z$.

$$
\begin{aligned}
& \iint_{0 \leq v \leq w^{r}<\infty} v^{\xi_{1}-1} w^{\xi_{3}-1} e^{-d_{1} v-d_{2} w}(-\ln w) d v d w \\
& \quad=-\frac{\partial}{\partial \xi_{3}} \iint_{0 \leq v \leq w^{r}<\infty} v^{\xi_{1}-1} w^{\xi_{3}-1} e^{-d_{1} v-d_{2} w} d v d w \\
& \quad=-\frac{\partial}{\partial \xi_{3}} \int_{0}^{1} z^{\xi_{1}-1} d z \int_{0}^{\infty} w^{\xi_{1} r+\xi_{3}-1} e^{-d_{1} w^{r} z-d_{2} w} d w \\
& =-\frac{\partial}{\partial \xi_{3}}\left(d_{1}^{-\xi_{1}} d_{2}^{-\xi_{3}} \kappa\left(\xi_{1}, \xi_{3}, r, \frac{d_{1}}{d_{2}^{r}}\right)\right) \\
& \quad=d_{1}^{-\xi_{1}} d_{3}^{-\xi_{3}}\left(\kappa\left(\xi_{1}, \xi_{3}, r, \frac{d_{1}}{d_{2}^{r}}\right) \ln d_{2}-\frac{\partial}{\partial \xi_{3}} \kappa\left(\xi_{1}, \xi_{3}, r, \frac{d_{1}}{d_{2}^{r}}\right)\right) .
\end{aligned}
$$

Proof of Theorem 2.3 Lemma 3.2 and Lemma 5.3 imply $\mathbf{E} X\left(d_{1}, d_{2}\right)=g\left(\alpha+\gamma, d_{1}, d_{2}\right)+$ $O_{d_{1}, d_{2}}(1)$. It remains to apply Lemmas 5.6, 5.7, and 5.8 to the definition of $g$.

\section{CONCENTRATION FOR NUMBER OF EDGES}

Proof of Theorem 2.4 We use the Azuma-Hoeffding inequality.

Theorem 6.1. [2, 13] Let $\left(X_{s}\right)_{s=0}^{n}$ be a martingale with $\left|X_{s+1}-X_{s}\right| \leq \delta$ for $s=$ $0, \ldots, n-1$, and $x>0$. Then,

$$
P\left(\left|X_{n}-X_{0}\right| \geq x\right) \leq 2 \exp \left(-\frac{x^{2}}{2 \delta^{2} n}\right) .
$$

We fix $d_{1}, d_{2}, t$ and denote $X=X\left(t, d_{1}, d_{2}\right)$. Let $G$ be a random graph in $\mathcal{G}(t)$; it has $t$ edges, sorted by the creation time. Let $G^{(s)}$ be a graph with $s$ first edges. Let $X_{s}=\mathbf{E}\left(X \mid G^{(s)}\right), s=t_{0}, \ldots, t$. In this sequence $X_{t_{0}}=\mathbf{E} X, X_{t}=X$. By definition of the probabilistic space, the sequence $X_{s}$ is a martingale. We will estimate possible differences between adjacent elements of the sequence.

We fix any $s$ from 0 to $t-1$. Let $v$ and $w$ be the source and the target of the last edge in $G^{(s+1)}$, so $v$ and $w$ are random quantities depending on $G$. Note that the pair $(v, w)$ also determines which one of processes $(\dagger),(\ddagger),(\dagger \dagger)$ was used. By definition

$$
\begin{aligned}
& X_{s}=\sum_{i, j} \operatorname{Pr}(v=i, w=j) \mathbf{E}\left(X \mid G^{(s)}, v=i, w=j\right), \\
& X_{s+1}=\quad \mathbf{E}\left(X \mid G^{(s)}, v=v\left(G^{(s+1)}\right), w=w\left(G^{(s+1)}\right)\right),
\end{aligned}
$$


where the sum is over all pairs of nodes of $G$. Hence, it is clear that

$$
\begin{gathered}
\min _{i, j} \mathbf{E}\left(X \mid G^{(s)}, v=i, w=j\right) \leq X_{s}, X_{s+1} \leq \max _{i, j} \mathbf{E}\left(X \mid G^{(s)}, v=i, w=j\right), \\
\left|X_{s}-X_{s+1}\right| \leq \max _{i, j} \mathbf{E}\left(X \mid G^{(s)}, v=i, w=j\right)-\min _{i, j} \mathbf{E}\left(X \mid G^{(s)}, v=i, w=j\right) .
\end{gathered}
$$

Let $\gamma_{1} \in \arg \min \mathbf{E}\left(X \mid G^{(s)}, v=i, w=j\right)$ and $\gamma_{2} \in \arg \max \mathbf{E}\left(X \mid G^{(s)}, v=i, w=j\right)$. It is sufficient to prove an upper bound for

$$
\mathbf{E}\left(X \mid G^{(s)}, v=i_{2}, w=j_{2}\right)-\mathbf{E}\left(X \mid G^{(s)}, v=i_{1}, w=j_{1}\right) .
$$

Replace the initial graph $G_{0}$ by $G^{(s)} \cup\left(i_{1}, j_{1}\right)$ and consider the quantity $E_{X}$ in that model; denote it as $E_{1}\left(T, N, d_{1}, d_{2}\right)$. Similarly, let $E_{2}\left(T, N, d_{1}, d_{2}\right)$ denote the analogue of $E_{X}$ in the model with the initial graph $G^{(s)} \cup\left(i_{2}, j_{2}\right)$. Then $\mathbf{E}\left(X \mid G^{(s)}, v=i_{1}, w=j_{1}\right)=$ $\mathbf{E}\left(E_{1}\left(t-s-1, N, d_{1}, d_{2}\right)\right)$ and $\mathbf{E}\left(X \mid G^{(s)}, v=i_{2}, w=j_{2}\right)=\mathbf{E}\left(E_{2}\left(t-s-1, N, d_{1}, d_{2}\right)\right)$, where $N$ has binomial distribution with parameters $t-s-1$ and $\alpha+\gamma$. We cannot use Theorem 2.3 for $E_{1}$ and $E_{2}$ directly because the remainder term of Theorem 2.3 depends on the initial graph, but (5.1) and (3.1) are still valid. Here, $A_{\text {in }}$ and $A_{\text {out }}$ can differ in models with initial graphs $G^{(s)} \cup\left(i_{1}, j_{1}\right)$ and $G^{(s)} \cup\left(i_{2}, j_{2}\right)$ because of the different number of vertices. However, a priori bounds $0 \leq n_{\text {in }}(t, d) \leq t$ and $0 \leq X\left(t, d_{1}, d_{2}\right) \leq t$ imply $E_{i}\left(T, N, d_{1}, d_{2}\right) \frac{d_{1}+\delta_{\text {out }}}{T+\delta_{\text {out }} N+A_{\text {out }}^{\prime}}=E_{i}\left(T, N, \bar{d}_{1}, d_{2}\right) \frac{d_{1}+\bar{\delta}_{\text {out }}}{T+\delta_{\text {out }} N+A_{\text {out }}}+O_{d_{1}, d_{2}}\left(\frac{1}{T}\right)$ (and similarly for other terms of (5.1) and (3.1)), so the difference $E_{1}\left(T, N, d_{1}, d_{2}\right)-E_{2}\left(T, N, d_{1}, d_{2}\right)$ satisfies to (5.1) with $E_{i n, d_{2}-1}(T, N-1)$ and $E_{\text {out }, d_{1}-1}(T, N-1)$ replaced by the corresponding differences, which in turn satisfy to (3.1) without terms $\frac{\alpha N}{(\alpha+\gamma)(T+1)}$ and $\frac{\gamma N}{(\alpha+\gamma)(T+1)}$.

It remains to note that adding an edge $\left(i_{1}, j_{1}\right)$ changes $X\left(t, d_{1}, d_{2}\right)$ by, at most, $d_{1}+d_{2}$, so the initial value $E_{1}\left(0,0, d_{1}, d_{2}\right)-E_{2}\left(0,0, d_{1}, d_{2}\right)$ is, at most, $2\left(d_{1}+d_{2}\right)$. Consequently, $E_{1}\left(T, N, d_{1}, d_{2}\right)-E_{2}\left(T, N, d_{1}, d_{2}\right)=O_{d_{1}, d_{2}}(1)$ similar to Lemma 5.3.

Therefore, the sequence $\left(X_{s}\right)$ satisfies the condition of Theorem 6.1 with $n=t-t_{0}$ and $\delta=O_{d_{1}, d_{2}}(1)$. Substituting $x=\sqrt{t} \ln t$ in Theorem 6.1, we obtain Theorem 2.4.

\section{REFERENCES}

[1] M. Abramowitz, and I. A. Stegun (editors). Handbook of Mathematical Functions with Formulas, Graphs and Mathematical Tables. New York: Dover, 1964, 10th GPO printing.

[2] K. Azuma. "Weighted Sums of Certain Dependent Variables." Tôhoku Math. J. 19 (1967), 357-367.

[3] A.-L. Barabási, and R. Albert. "Emergence of Scaling in Random Networks." Science 286 (1999), 509-512.

[4] B. Bollobás. Random Graphs (2nd ed.). Cambridge, UK: Cambridge Univ. Press, 2001.

[5] B. Bollobás, C. Borgs, J. Chayes, and O. Riordan. "Directed Scale-Free Graphs." In Proc. 14th ACM-SIAM Symposium on Discrete Algorithms, pp. 132-139. New York: ACM, 2003.

[6] B. Bollobás, O. M. Riordan, J. Spencer, and G. Tusnády. "The Degree Sequence of a Scale-Free Random Graph Process." Random Structures and Algorithms 18 (2001), N3, 279-290.

[7] P. G. Buckley, and D. Osthus. "Popularity Based Random Graph Models Leading to a Scale-Free Degree Sequence." Discrete Math. 282 (2004), 53-68.

[8] C. Cooper. "Distribution of Vertex Degree in Web-Graphs." Combinatorics Probability and Computing 15 (2006), 637-661.

[9] C. Cooper, and A. Frieze. "A General Model of Web Graphs." Random Structures and Algorithms 22 (2003), 311-335. 
[10] S. N. Dorogovtsev, J. F. F. Mendes, and A. N. Samukhin . "Structure of Growing Networks with Preferential Linking." Phys. Rev. Lett. 85 (2000), 4633.

[11] E. Drinea, M. Enachescu, and M. Mitzenmacher. "Variations on random graph models for the web." Technical Report, Harvard University, Department of Computer Science, 2001.

[12] E. A. Grechnikov. "The Degree Distribution and the Number of DEdges Between Vertices of Given Degrees in the Buckley-Osthus Model of a Random Web Graph." J. of Internet Mathematics 8 (2012), 257-287.

[13] W. Hoeffding. "Probability Inequalities for Sums of Bounded Random Variables." J. Amer. Statist. Assoc. 58 (1963), 13-30.

[14] S. Janson, T. Łuczak, and A. Ruciński. Random Graphs. New York: Wiley, 2000. 